\title{
902-928MHz UHF RFID Tag Antenna Design, Fabrication and Test
}

\author{
A Thesis \\ presented to \\ the Faculty of California Polytechnic State University, \\ San Luis Obispo
}

\author{
In Partial Fulfillment \\ of the Requirements for the Degree \\ Master of Science in Electrical Engineering
}

by

ChiWeng Kam

August 2011 
(C) 2011

ChiWeng Kam

ALL RIGHTS RESERVED 


\section{COMMITTEE MEMEBERSHIP}

TITLE: $\quad$ 902-928MHz UHF RFID Tag Antenna Design, Fabrication and Test

AUTHOR:

DATE SUBMITTED:

COMMITTEE CHAIR:

COMMITTEE MEMBER:

COMMITTEE MEMBER:
ChiWeng Kam

August 2011

Dr. Dean Arakaki

Associate Professor of Electrical Engineering, Advisor

Dr. Dennis Derickson

Chair, Department of Electrical Engineering

Dr. Vladimir Prodanov

Assistant Professor of Electrical Engineering 


\section{ABSTRACT \\ 902-928MHz UHF RFID Tag Antenna Design, Fabrication and Test ChiWeng Kam}

Radio Frequency Identification (RFID) uses RF radiation to identify physical objects. With decreasing integrated circuit (IC) cost and size, RFID applications are becoming economically feasible and gaining popularity. Researchers at MIT suggest that RFID tags operating in the $900 \mathrm{MHz}$ band (ultrahigh frequency, UHF) represent the best compromise of cost, read range, and capabilities [1]. Passive RFID tags, which exclude radio transmitters and internal power sources, are popular due to their small size and low $\operatorname{cost}[1]$.

This project produced Cal Poly's first ever on-campus printed, assembled, and operational UHF (902 to $928 \mathrm{MHz}$ ) passive RFID tag. Project goals include RFID tag antenna design and simulation using the EMPro electromagnetic (EM) simulation tool [47], establishing the tag fabrication process, and testing, operational verification, and comparisons to commercial tag performance. The tag antenna design goal is to meet or exceed the read range performance of the commercial Sirit tag [23] while minimizing the required tag conductive area.

This thesis provides an overview of the UHF passive RFID tag fabrication process. Cal Poly's Graphic Communication Department Laboratory applied a screen-printing process to print RFID tag antenna patterns onto plastic (PET) substrates. RFID IC-substrate packages were manually attached to tag antennas with conductive adhesives and functionally verified and compared to commercial tag performance. 
RFID tag antennas were impedance matched (using EMPro) to the Monza 3 RFID IC to maximize IC to antenna power transfer and RFID tag read range.

Tag antenna read range (maximum reader-tag communication distance) was

characterized in Cal Poly's Anechoic Chamber, while RFID tag matching characteristics were measured using the differential probe method [33-41] and compared to simulations. Read range results indicate that one of the designs developed in this thesis outperforms a commercial UHF RFID tag. 


\section{ACHKNOWLEDGMENTS}

I want to specially thank Dr. Dean Arakaki for his guidance and all the time put into reviewing my work. I want to also thank Dr. Derickson and Dr. Prodanov for taking the time to serve on my thesis committee.

This project would not be possible without the support from Dr. Tali Freed (Industrial and Manufacturing Engineering Department) and Dr. Xiaoying Rong (Graphic Communication Department). Thank you to the material support and advice provided by Wolf Bielas and Candace Vanderbilt at the Sirit Inc. Thanks to Dr.Daniel Deavours at University of Kansas for the helpful advices.

I also want to thank Benafsh Husain for all her motivation and positive encouragement during this entire thesis process. She provides me with consistent support to work this thesis through.

Finally, I want to dedicate this thesis to my family who provides all the possible support and resource for me to study in U.S.A. I want to thank the Electrical Engineering Department at CalPoly for the wonderful university experience and knowledge that will serve me well in my future career. 


\section{TABLE OF CONTENTS}

LIST OF TABLES ....................................................................................................... viii

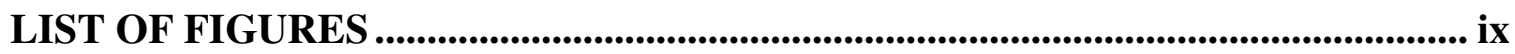

1 INTRODUCTION .........................................................................................................1

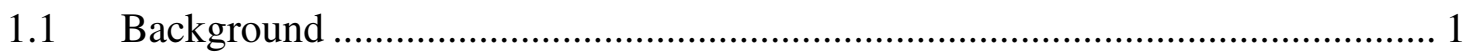

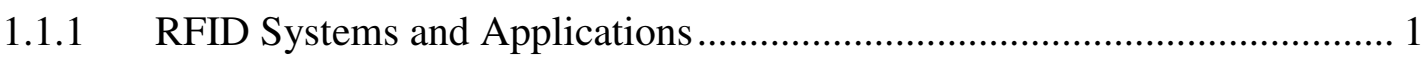

1.1.2 Introduction to UHF RFID System Components......................................... 5

1.2 Project Objectives and Organization.......................................................... 14

2 UHF RFID TAG FABRICATION...............................................................16

$2.1 \quad$ Fabrication Process Overview.................................................................. 16

2.1.1 Antenna Fabrication........................................................................... 17

2.1.2 IC-Antenna Attachment Procedures ......................................................... 21

2.1.3 Inlay Post-Processing ......................................................................... 24

3 UHF RFID ANTENNA DESIGN ..........................................................................25

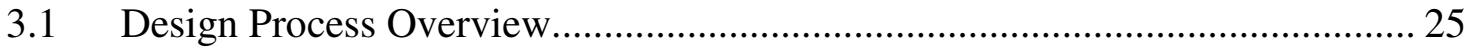

3.1.1 Tag Design Requirements.............................................................. 25

3.1.2 Antenna Construction Materials and Methods …………………………..... 29

3.1.3 Packaged ASIC RF Impedance Measurement ............................................ 29

3.1.4 Antenna Type Identification and Design Techniques.................................. 32

3.1.5 Tag Performance Simulation and Optimization ......................................... 40

3.1.6 Prototype Construction and Test........................................................... 50

4 UHF RFID TAG OPERATION VERIFICATION .............................................64

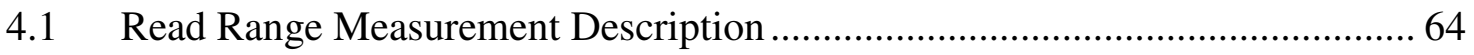

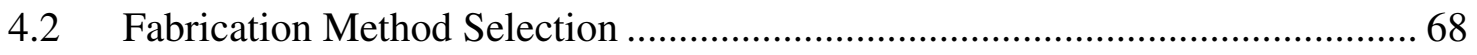

4.3 RFID Tag Design E- and H-Plane Patterns................................................... 72

4.4 Patch Antenna Gain Extraction .............................................................. 76

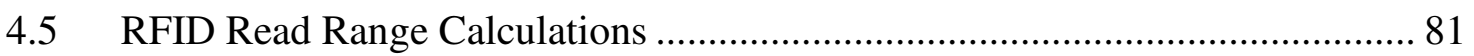

5 CONCLUSIONS AND FUTURE RECOMMENDATIONS..................................84

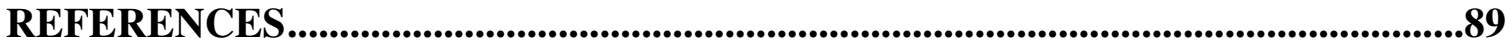

Appendix A: RFID System Operating Frequency Comparison......................................93

Appendix B: UHF RFID System Protocols and Anti-Collision Algorithms................95

Appendix C: RFID Antenna Fabrication Techniques [15] ...............................................97

Appendix D: Screen Printing File Formats ..............................................................................99 


\section{LIST OF TABLES}

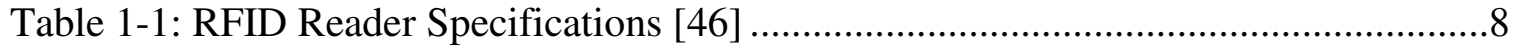

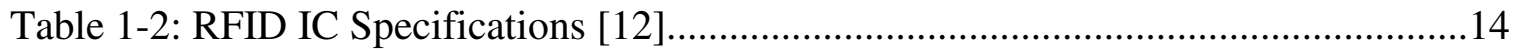

Table 3-1: UHF RFID Tag Fabrication Materials ........................................................29

Table 3-2: RFID IC Impedance Measurement................................................................... 31

Table 3-3: Monza 3 IC Recommended Antenna Input Impedance ...............................48

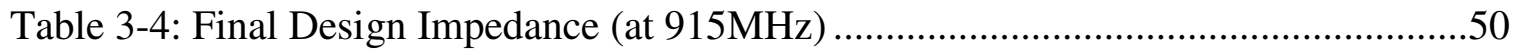

Table 3-5: Final Tag Designs Key Structural Dimensions $(\mathrm{mm})$................................50

Table 4-1: Tag and Patch Antenna Alignment: Chamber (unprimed), Tag (primed) and Patch Antenna (double-primed) Coordination Systems [42] ....................68

Table 4-2: Attenuation vs. Measured RF Power, ALR-9800 RFID Reader [42] .............68

Table 4-3: Fabricated Tag Configuration ................................................................69

Table A-1: RFID System Frequency Band Classification [43] ...................................93

Table A-2: RFID Tag Powering Classification [1] .....................................................94

Table B-1: RFID Reader Protocols and Anti-Collision Algorithms...............................95 


\section{LIST OF FIGURES}

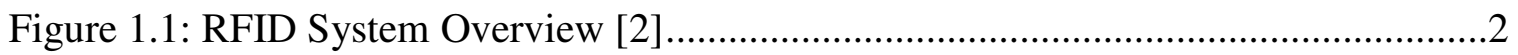

Figure 1.2: Common RFID Bands [1] .......................................................................

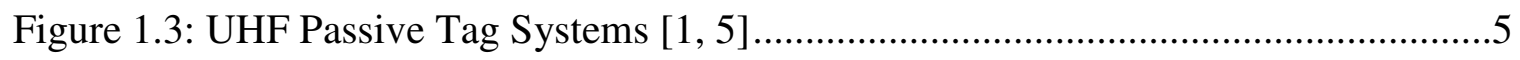

Figure 1.4: On-Off Keying Scheme [1] .................................................................

Figure 1.5: Pulse-Interval Encoding Scheme [1] ........................................................

Figure 1.6: Linearly Polarized Radiation [1] ................................................................

Figure 1.7: Circularly Polarized Radiation [1] ..........................................................

Figure 1.8: Polarization Alignment and Tag-Reader Visibility ........................................

Figure 1.9: RFID Reader Patch Antenna ………………….........................................10

Figure 1.10: RFID Application; After Crump, US Patent 2,943,189, Filed 1956,

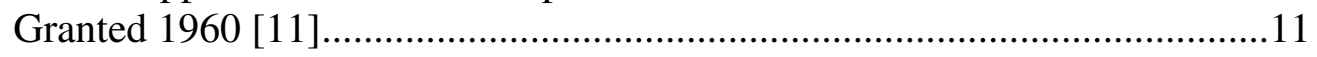

Figure 1.11: Inductively Coupled Identification System Example [1] ............................12

Figure 1.12: Passive RFID Tag Schematic [1] ............................................................13

Figure 1.13: (a) Commercial Passive UHF Tag (Alien Technology Model 9238

'Squiggle') (b) Strap Package.........................................................................13

Figure 2.1: (a) Smart-Label Configurations and (b) Manufacturing Process [1] ...............16

Figure 2.2: Screen Printing, A: Ink, B: Roller or Squeegee, C: Open Area, D: InkBlocking Stencil, E: Mesh Polyester Screen, F: Substrate ...............................18

Figure 2.3: RFID Antenna Positive Photoresist Pattern ...................................................19

Figure 2.4: RFID Antenna Print Screen...................................................................19

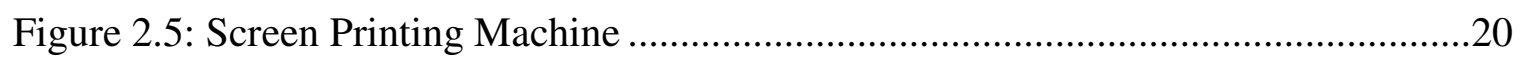

Figure 2.6: Roller Over Stencil Pattern.................................................................20

Figure 2.7: Printed RFID Tag Antenna...................................................................21

Figure 2.8: IC-Antenna Attachment Techniques (a) Wire Bonding, (b) Flip Chip...........22

Figure 2.9: IC-Antenna Attachment using Anisotropic Conductive Adhesives [17, 18] .22

Figure 2.10: IC-Antenna Attachment Using Strap Package and Conductive Tape ...........23

Figure 2.11: Fluidic Self-Assembly (FSA)-Assembled Strap Cross-Section [15] ............24

Figure 3.1: RFID Tag Antenna Design Flow Graph..................................................25

Figure 3.2: Antenna Impedance and Chip Impedance vs. Frequency for a Typical

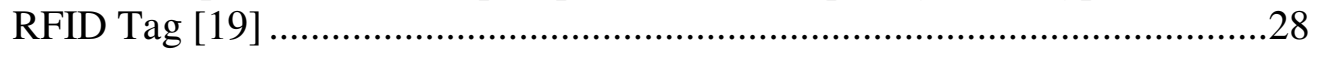

Figure 3.3: RFID IC (Strap) on ICM TF-30001-B Test Fixture.........................................30

Figure 3.4: RFID IC \#1 Impedance Measurement...........................................................30 


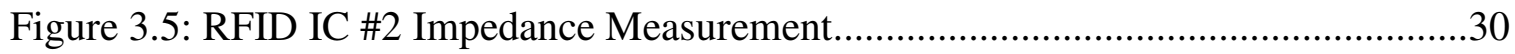

Figure 3.6: RFIC Measured Impedance vs. Frequency ..................................................31

Figure 3.7: EMPro Model, Sirit RSI-674 Inlay RFID Tag .........................................32

Figure 3.8: Sirit RSI-674 UHF RFID Tag Design Techniques .....................................32

Figure 3.9: Meandered Dipole with Reduced Projected Length [15] ..............................33

Figure 3.10: Tip-loaded Dipole with Reduced Projected Length [15] .............................34

Figure 3.11: Fat Dipole with Reduced Projected Length [15] ......................................34

Figure 3.12: Meander Dipole Impedance Matching Structures (a) Inductively Coupled Loop [25], (b) Loading Bar [25], (c) T-Matching Structure [15] .35

Figure 3.13: (a) Dipole with T-Matching Structure, (b) Equivalent Circuit [22] .............36

Figure 3.14: T-Matching Dimension Matching Chart (a) From Ref [25] (b)

Reproduced. .38

Figure 3.15: (a) T-Matching, Inductor-Based View (b) Proposed Passive UHF Tag

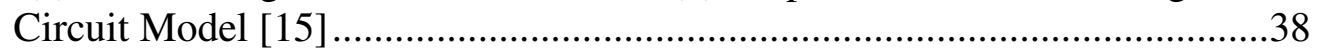

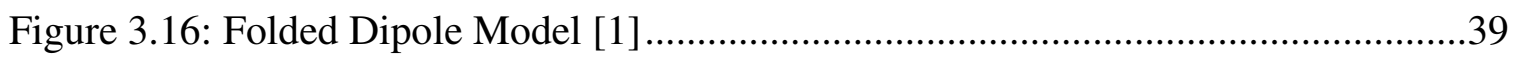

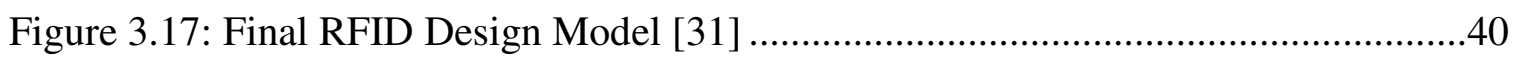

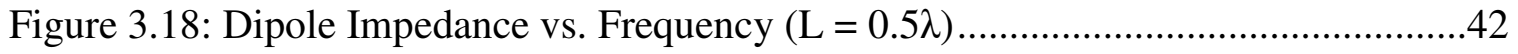

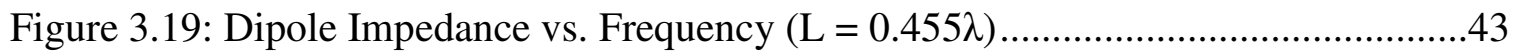

Figure 3.20: Folded Dipole Impedance vs. Frequency ..............................................44

Figure 3.21: Folded Dipole, 25 mm Folded Length ...................................................45

Figure 3.22: Resistance vs. Frequency, Multiple Folded Dipole Lengths.......................45

Figure 3.23: Reactance vs. Frequency, Multiple Folded Dipole Lengths .......................46

Figure 3.24: EMPro Model, Closed Loop Structure .....................................................46

Figure 3.25: Extracted Sirit RFID Tag Impedance Frequency Response ........................47

Figure 3.26: Tag Design Input Impedance Frequency Responses (a) Design \#1, (b) Design \#2, (c) Design \#3

Figure 3.27: Final RFID Antenna Designs (a) Design \#1, (b) Design \#2, (c) Design \#3 .50

Figure 3.28: RFID Strap and Virtual Ground Plane [38] .51

Figure 3.29: RFID Impedance Measurement, Commercially Available SingleEnded Probe (V= voltage) [39] .52

Figure 3.30: RFID Impedance Measurement, Commercial Wire Balun [39]. .53

Figure 3.31: Differential Method Test Fixture (a) Classic Fixture [34] (b) Modern Fixture \#1 [40] (c) Modern Fixture \#2 [40].... .54 
Figure 3.32: RFID S-Parameter Measurement Setup ................................................55

Figure 3.33: RFID Tags Fabricated on FR4 board and Test Fixture ..............................56

Figure 3.34: Test Fixture, Short Circuit Configuration ............................................57

Figure 3.35: $\mathrm{S}_{11}$ (a) Without Port Extension and (b) With Port Extension.......................57

Figure 3.36: (a) Tag Antenna Under Test Mounted on Test Fixture (b)

Measurement Setup Inside Anechoic Chamber ..........................................58

Figure 3.37: Sirit Tag Impedance, Measured vs. Simulated (a) Resistance (b)

Reactance.

Figure 3.38: Tag Design \#1 Impedance, Measured vs. Simulated (a) Resistance (b)

Reactance.

Figure 3.39: Tag Design \#2 Impedance, Measured vs. Simulated (a) Resistance (b)

Reactance.

Figure 3.40: Tag Design \#3 Impedance, Measured vs. Simulated (a) Resistance (b)

Reactance.

Figure 4.1: Original Chamber Configuration [42].....

Figure 4.2: Modified Chamber Configuration (Tag Center to TX/RX Pair Center Alignment)..... .66

Figure 4.3: RFID Tag Fixture, H-Plane Co-pol

Figure 4.4: RFID Tag Fixture, E-Plane Co-pol

Figure 4.5: Test Configuration (a) Tag Coordinate System [42] (b) Patch Antenna Coordinate System. .67

Figure 4.6: Angular Relationship, TX or RX Antenna and Tag Centers.

Figure 4.7: Sirit Tag E- and H-Plane Patterns, E- and H-Plane X-pol: OdB (Not Plotted) .70

Figure 4.8: E- and H-Plane Pattern Comparison (Conductive vs. Scotch Tape) ...............71

Figure 4.9: E- and H-Plane Pattern Comparison (Laminated vs. Non-Laminated) ...........72

Figure 4.10: Tag Design \#1 E- and H-Plane Patterns, E- and H-Plane X-pol: OdB (Not Plotted)

Figure 4.11: Tag Design \#2 E- and H-Plane Patterns, E- and H-Plane X-pol: OdB (Not Plotted)

Figure 4.12: Tag Design \#3 E- and H-Plane Patterns, E- and H-Plane X-pol: OdB (Not Plotted). .75

Figure 4.13: E- and H-Plane Pattern Comparison .........................................................76

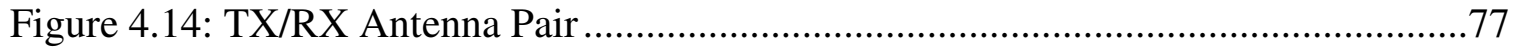

Figure 4.15: EMPro TX/RX Patch Antenna Models (a) Patch \#1 (b) Patch \#2 (Eplane: YZ).... 
Figure 4.16: Simulated Patch TX/RX Antenna $\left|S_{11}\right|$ vs. Frequency ................................78

Figure 4.17: Radiation Pattern Phi Cut $\left(\varphi=90^{\circ}\right)$ E-Plane Co-Pol (a) Patch \#1 (b) Patch \#2

Figure 4.18: Radiation Pattern Phi Cut $\left(\varphi=90^{\circ}\right)$ Rectangular Plot ...............................79

Figure 4.19: Radiation Pattern Phi Cut $\left(\varphi=0^{\circ}\right)$ H-Plane Co-Pol (a) Patch \#1! (b)

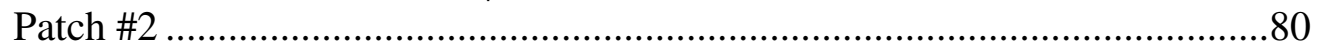

Figure 4.20: Angular Relationship, TX Antenna and Tag..........................................80

Figure 4.21: RF Transmit Power (W) and Read Range (m) vs. Reader Attenuation

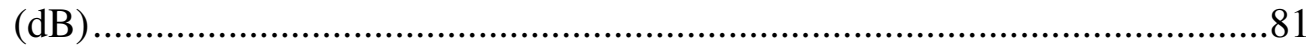

Figure 4.22: RFID Tag Read Range (a) Rectangular Plot (b) Read Range Pattern...........82

Figure 5.1: Measured RFID Antenna Resistance (Controlled vs. Uncontrolled Environment) .86

Figure 5.2: Measured RFID Antenna Reactance (Controlled vs. Uncontrolled Environment).

Figure 5.3: Simulated RFID Antenna Resistance, Silver Ink on PET vs. Copper on FR4

Figure 5.4: Simulated RFID Antenna Resistance, Silver Ink on PET vs. Copper on FR4 .88

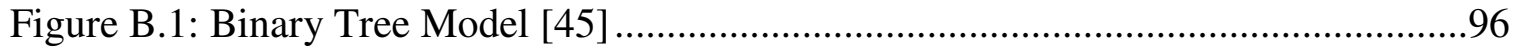

Figure B.2: Binary Tree and DFSA Algorithm Comparison [45] .96 


\section{INTRODUCTION}

\subsection{Background}

This section provides a comprehensive overview and introduction to RadioFrequency Identification (RFID) systems. Section 1.1.1 discusses RFID system applications and three methods of categorizing RFID systems: operating frequency band, transponder (tag) powering techniques, and communication protocols - rules that govern communications between the interrogator (reader) and transponder. Section 1.1.2 focuses on UHF (ultra-high frequency) RFID systems and its three main components: the interrogator, tag-reader communications, and the transponder (tag). This section also provides information about test equipment used in this project.

\subsubsection{RFID Systems and Applications}

Radio-Frequency Identification (RFID) is the use of RF radiation to identify physical objects. Automated identification systems include RFID and bar code systems. Unlike bar code systems, RFID systems eliminate "line-of-sight to object" requirements. Figure 1.1 illustrates a simplified RFID system. The system uses radio reader-tag transmissions to identify a tagged object. Each RFID tag contains an identification number. The RFID reader detects tags through RF radiation backscattered from RFID tags. The tag system rectifies the received RF signal to power the tag circuitry and send a tag identification signal to the reader [1]. 


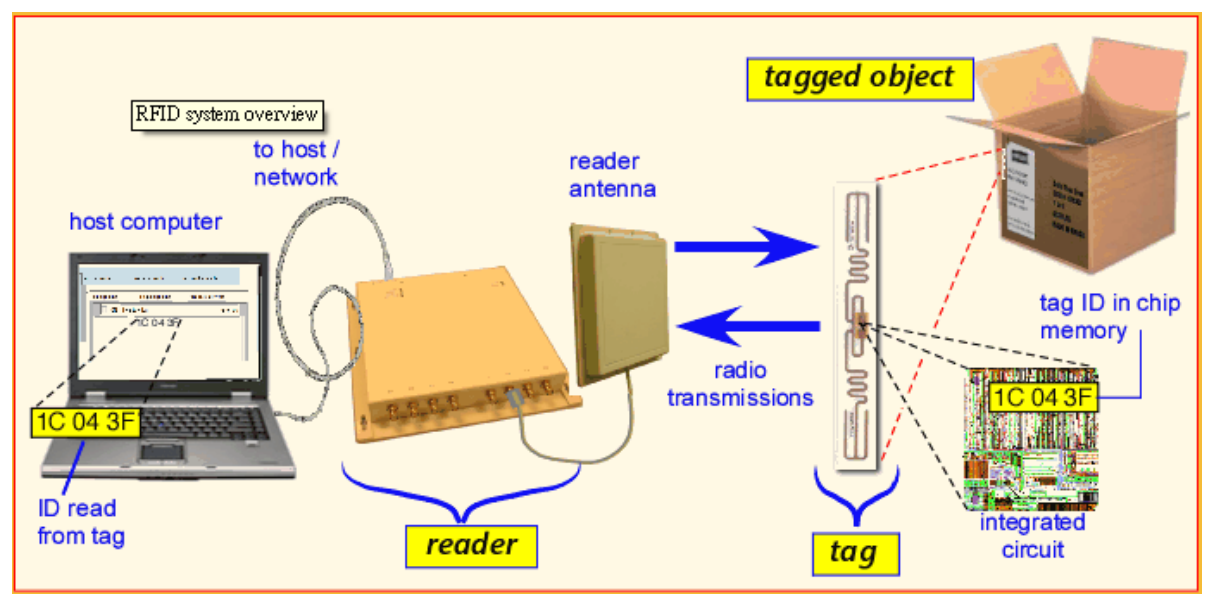

Figure 1.1: RFID System Overview [2]

RFID systems are categorized by operating frequency bands, tag powering techniques, and tag-reader communication protocols. These aspects help define read range, cost, and available features.

Figure 1.2 lists frequency bands commonly used in RFID systems. LF (125 and $134 \mathrm{kHz}$ ) and HF (13.56 MHz) RFID systems utilize inductive coupling (Section 1.1.2.2) with typical read ranges less than $60 \mathrm{~cm}$. UHF RFID has read range up to $3 \mathrm{~m}$. Microwave (e.g.: 2.4 GHz) RFID systems with radiative coupling (Section 1.1.2.2) have read ranges of approximately $1 \mathrm{~m}$ due to environmental effects, i.e.: microwave RFID cannot penetrate water and metal (see Appendix A for an RFID system operating frequency comparison). Along with UHF tags designs that operate near metal and high water content surfaces, UHF RFID systems are gaining popularity [3]. 


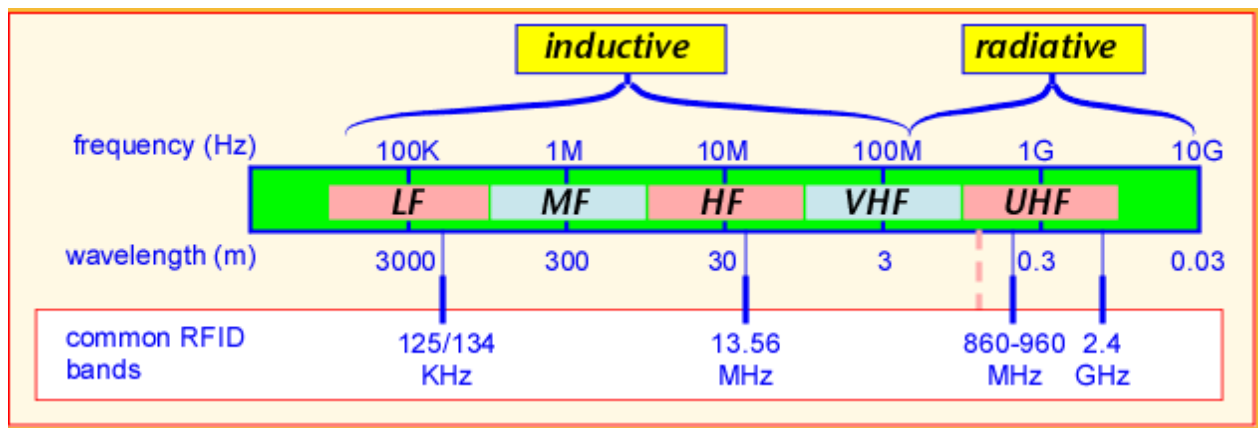

Figure 1.2: Common RFID Bands [1]

There are three types of RFID tags - passive, semipassive and active tags. A passive tag derives power from the reader's radio signals, whereas an active tag contains its own power source and conventional transmitter. Semipassive tags, also known as battery-assisted passive tags, include internally-powered electronic circuitry, but no transmitter (see Appendix A for a tag-powering technique comparison). Passive RFID tags are popular due to their small size and low cost compared to active and semipassive tags.

Communication protocols define conventions and industry standards on information exchange formats between tags and readers including symbol bit coding, carrier frequency range and modulation methods, symbol packet organization, and medium allocation [2]. Communication standards are established by the Institute of Electrical and Electronic Engineers (IEEE), EPCglobal Inc (specifically for RFID), the American National Standards Institute (ANSI), and the International Organization for Standardization (ISO), to ensure common protocols among all manufacturers and vendors. The Auto-ID Center was launched at MIT (1999) to promote RFID systems [1]. Their research suggests that RFID tags operating in the $900-\mathrm{MHz}$ band represent the best compromise of cost, read range, and capabilities [1]. EPCglobal Inc. was formed in 2003 
to support supply-chain RFID standards and established a new communication protocol, Class 1 Generation 2, for UHF RFID systems. The Gen2 protocol is becoming a globally accepted standard for passive RFID tags [1].

With decreasing integrated circuit cost and size, RFID applications are becoming economically feasible and gaining popularity. Early RFID applications involving large asset tracking began in the railroad industries (railcar tracking). Livestock management and shipping container tracking became popular due to large numbers of assets. In the 1990's, the tracking of people using RFID systems became possible. RFID systems were also used in retail supply chains to track items ranging from library books to beer kegs. In 2005, Wal-Mart, the world's largest retailer at that time, required its top 100 suppliers to include RFID tags on all cases and pallets delivered to Wal-Mart [1].

Another important application is the healthcare industry, including hospitals and clinics. According to BlueBean (RFID vendor), hospitals overstock 20-30\% of their mobile assets due to inventory mismanagement. Nursing department spend $10-30 \%$ of their work time searching for equipment, while equipment servicing departments spend $75 \%$ of the time locating each item [4]. Hospitals encounter patient billing difficulties due to consumable item tracking problems in the emergency room. As a result, asset management and patient tracking systems are helpful in reducing cost and increasing hospital efficiency.

As mentioned previously, UHF RFID systems with passive tags have generated substantial research interest, i.e.: the new EPCglobal standard. UHF tags enable relatively long read ranges compared to LF and HF tags. A UHF RFID system with passive tags is the focus of this project. 


\subsubsection{Introduction to UHF RFID System Components}

This section introduces UHF RFID system components. Figure 1.3 illustrates an early UHF passive tag system built in 1975 by Koelle et al., which also applies to many modern UHF RFID system features $[1,5]$. The tag is powered by rectifying, filtering, and regulating the incoming RF received signal through a diode, capacitor, and regulator. The antenna's load impedance is modified by a load modulator to send a coded signal back to the reader. An oscillator is used to implement a subcarrier modulation scheme; the tag antenna impedance is switched to produce a binary ' 0 ' (relatively small degree of antenna impedance modulation) and binary ' 1 ' (large modulation). In this implementation, an ID space (number of bits used for tag identification) of three bits maximum is provided by the ID code generator due to the circuit's maximum allowed power consumption. The reader uses a homodyne (single frequency) detection scheme in which the transmitted and received signals are combined to form the information signal $[1,5]$.

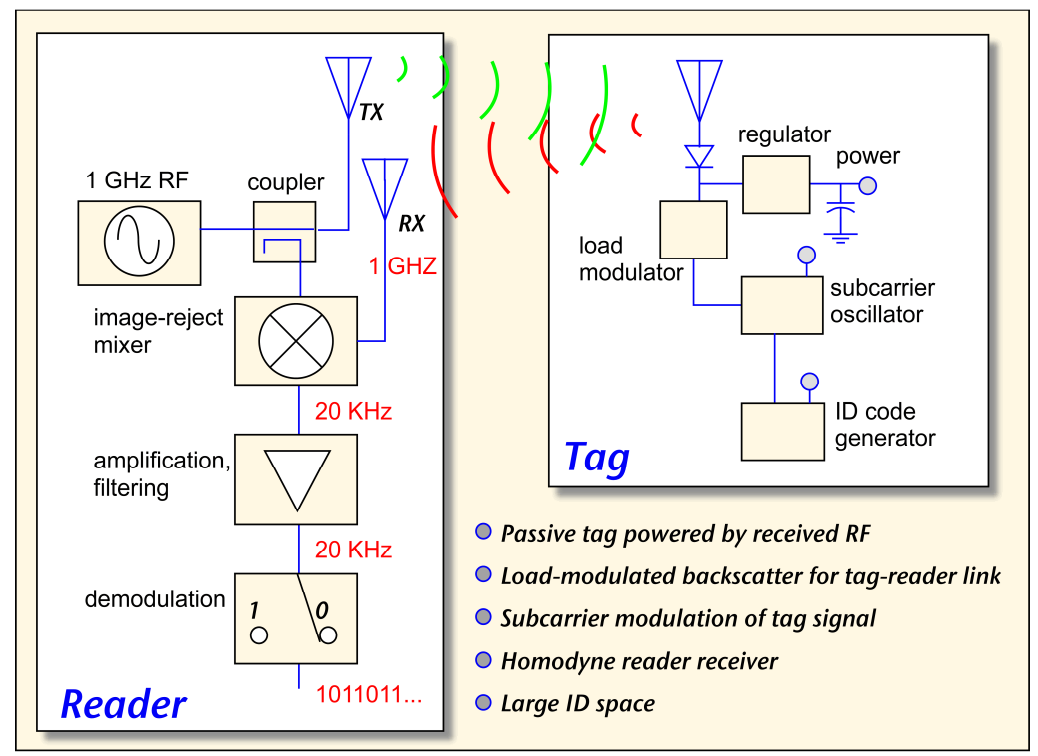

Figure 1.3: UHF Passive Tag Systems $[1,5]$ 
In UHF passive tag systems, reader antennas may be integrated with the reader or physically separated and connected by cable. Reader and antenna systems are described in Section 1.1.2.1. Section 1.1.2.2 describes reader-tag communications while Section 1.1.2.3 provides an overview of UHF RFID Tags. The antenna and integrated circuit (IC) which stores the tag ID and the logic necessary to execute the tag-reader communication protocols are also described.

\subsubsection{RFID Reader and Reader Antenna}

This section includes a brief description of basic UHF RFID system modulation and coding, anti-collision and multiple access techniques. The RFID reader system and antenna specifications in this project are briefly described.

Wireless communications typically utilize modulation schemes in which low frequency baseband information modulates a high frequency carrier for more effective propagation through a communication medium. The low operating power of passive RFID tags requires simplified modulation schemes for tag-reader communications. RFID signals are generally digitally modulated using amplitude modulation; the signal is represented by a stream of distinct symbols. On-off keying (OOK) scheme, in which high signal power indicates a binary ' 1 ' and small or zero signal power for a binary ' 0 ', can be implemented by a switch, such as a transistor, between the antenna and load. Fixed duration symbols for which signal power is either high or low are shown in Figure 1.4 [1]. 


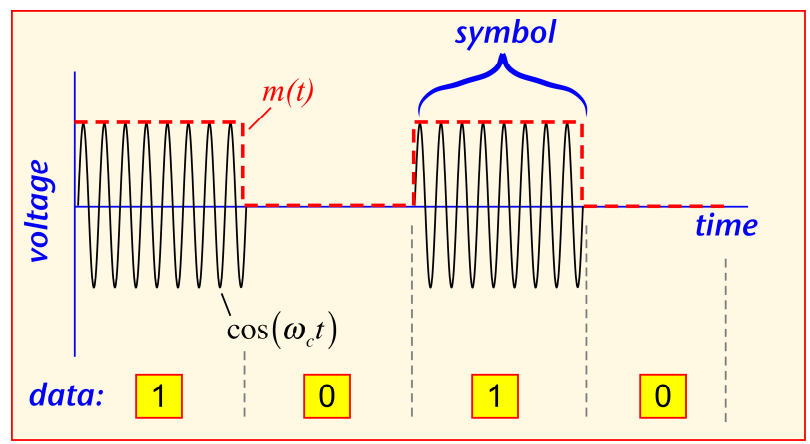

Figure 1.4: On-Off Keying Scheme [1]

A major drawback of the OOK method in passive RFID tags occurs for consecutive binary '0' symbols. With no available power, the tag cannot power its circuitry. Hence, a common solution is to code the binary data prior to modulation, e.g.: pulse-interval encoding (PIE). As illustrated in Figure 1.5, a binary ' 1 ' is represented by a long full-power pulse followed by a short power-off pulse. A binary ' 0 ' is coded as short full-power pulse and the same duration power-off pulse. This scheme, adopted by the EPCglobal Class 1 Generation 2 standard, allows at least 50\% maximum power delivery to the tag. Demodulation for both OOK and PIE schemes is accomplished through a diode-capacitor envelope detector [1] to recover the transmitted digital data.

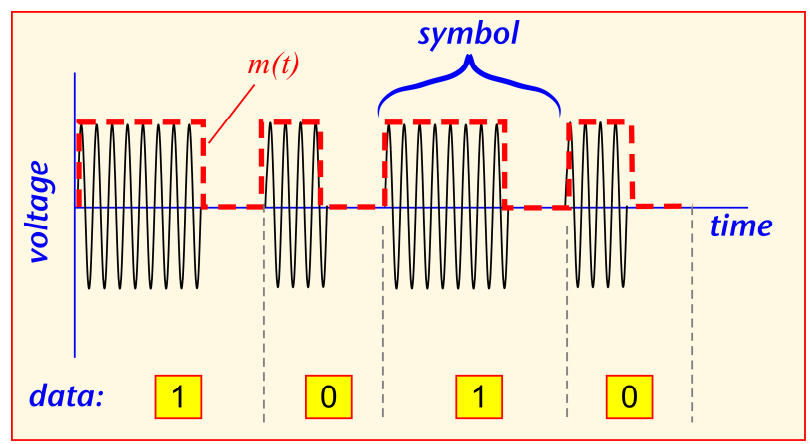

Figure 1.5: Pulse-Interval Encoding Scheme [1] 
Another key aspect of RFID readers is multiple access methods that allow multiple channel communication in a single medium. There are three basic multiplexing techniques - frequency-division multiple access (FDMA), time-division multiple access (TDMA) and code-division multiple access (CDMA). In FDMA, each user is assigned a unique carrier frequency. TDMA allows multiple users in a common frequency band separated by time slots. CDMA assigns unique pseudorandom binary codes to each user. If several tags within the reader's range attempt to send signals to the reader simultaneously, interference (i.e.: collision) occurs at the reader. Consequently, RFID systems typically employ the TDMA anti-collision method to poll tags individually. RFID system performance is often evaluated by its anti-collision algorithm effectiveness. Appendix B compares anti-collision algorithms for multiple UHF RFID system communication protocols. Several authors propose using CDMA-based anti-collision algorithms due to unique codes assigned to each tag [6-8]. Table 1-1 shows the RFID reader specifications used in this project.

Table 1-1: RFID Reader Specifications [46]

\begin{tabular}{|l|l|}
\hline Model Number & ALR 9800, Alien Technology \\
\hline $\begin{array}{l}\text { Supported RFID Tag } \\
\text { Protocols }\end{array}$ & EPC Gen2; ISO 18000-6c \\
\hline Anti-Collision Algorithm & "Q"-protocol based on DFSA \\
\hline Reader Protocols & Alien Reader Protocol, SNMP, firmware upgradable \\
\hline Frequency & $902.75 \mathrm{MHz}-927.25 \mathrm{MHz}$ \\
\hline RF Power & Max 4W EIRP with Alien Antenna \\
\hline Communications & RS-232 (DB-9 F), LAN TCP/IP $(\mathrm{RJ}-45)$ \\
\hline Antennas & $\begin{array}{l}\text { 4 ports; multi-static topology; circular or linear } \\
\text { polarization, reverse polarity TNC; requires minimum of } 2 \\
\text { antennas or external circulator }\end{array}$ \\
\hline Operating temperature & $-20^{\circ} \mathrm{C}$ to $+50^{\circ} \mathrm{C}\left(-4^{\circ} \mathrm{F}\right.$ to $\left.+122^{\circ} \mathrm{F}\right)$ \\
\hline Compliance Certification & $\begin{array}{l}\text { Emissions: FCC Part } 15 \\
\text { Safety: UL } 60950\end{array}$ \\
\hline
\end{tabular}


RFID reader antennas are directional; RF power is focused in one direction since RFID tags are generally located within relatively well-defined angular regions relative to the reader. Another factor in reader antenna design is antenna polarization. Propagating electric field direction determines radiated wave polarization. Figure 1.6 and Figure 1.7 illustrate two common polarizations, linear and circular. To transmit maximum power to the RFID tag, the tag and reader antennas must be polarization aligned. Perpendicular orientation results in no power transfer. Figure 1.8 demonstrates the polarization alignment and tag-reader visibility. As a result, the linearly polarized reader antennas are very sensitive to tag orientation compared to the circularly polarized reader antennas. However, half the power in circularly polarized reader antenna is transmitted relative to linearly polarized antenna pairs.
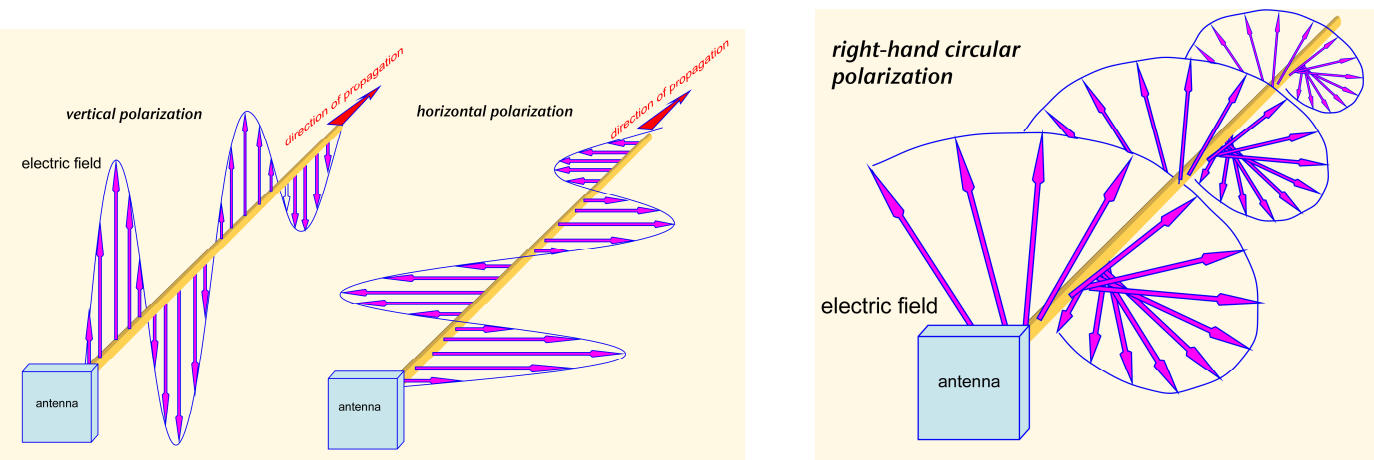

Figure 1.6: Linearly Polarized Radiation [1]

Figure 1.7: Circularly Polarized Radiation [1]

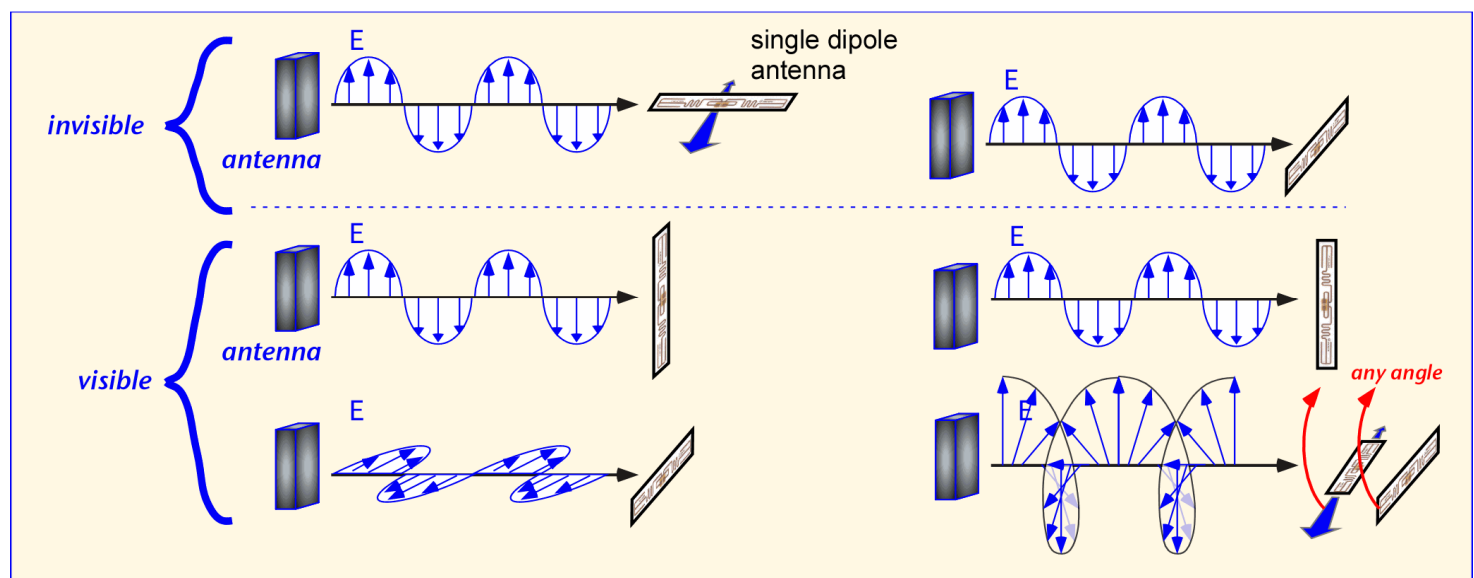

Figure 1.8: Polarization Alignment and Tag-Reader Visibility 
In this project, a pair of linearly polarized patch antennas was used as the RFID reader's transmit (TX) and receive (RX) antennas. The antennas are shown in Figure 1.9 and were previously designed to operate with the RFID reader (ALR-9800) [42].

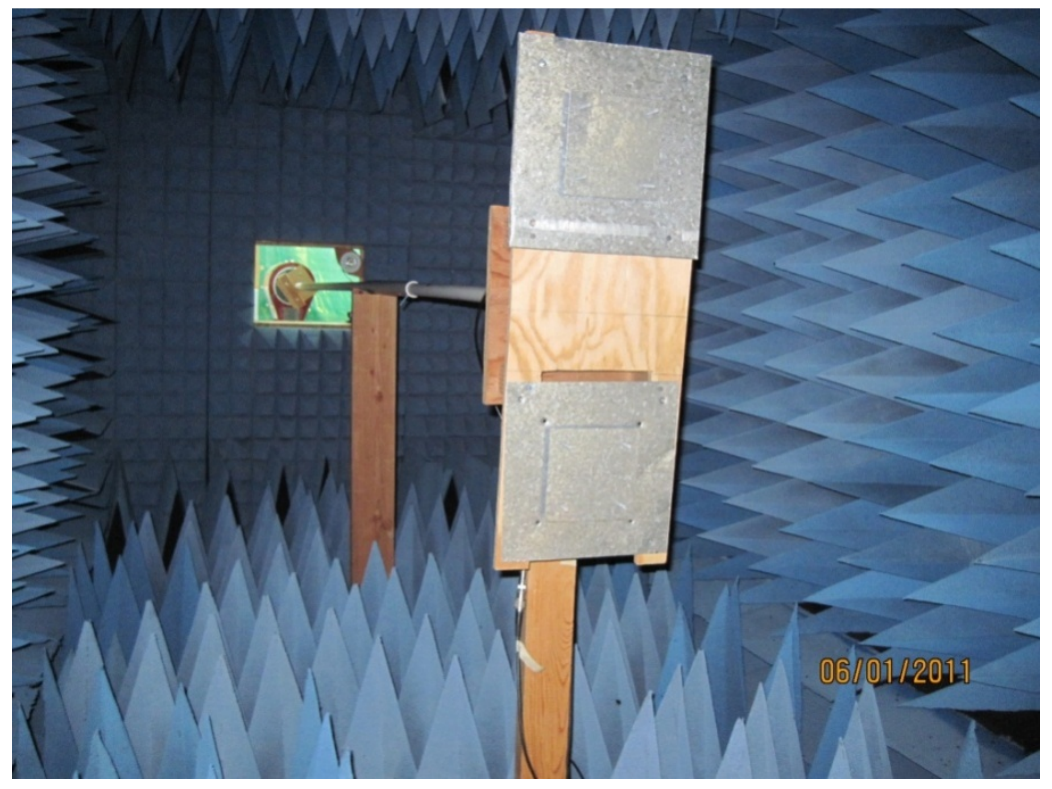

Figure 1.9: RFID Reader Patch Antenna

RFID coverage can be extended by implementing a four patch antenna phased array as the reader antenna [9]. A phased array is a group of antennas excited to dynamically direct a beam in a desired direction, known as beam scanning. Multiple access methods involving beam scanning are referred to as SDMA (Space-Division Multiple Access or Spatial Division Multiple Access). To access a communication channel, an SDMA system first identifies the user location, then establishes a one-to-one mapping between the network and user location. The one-to-one mapping can be established by using the phased array's narrow beam. 


\subsubsection{Reader and Tag Communications}

Low cost passive RFID systems use RFID tag backscattered radiation. By varying reflected signal properties, reader communication is possible without a high power transmitter in the RFID tag. The first example of a backscatter radio link was reported in 1948 [10]. An early RFID application appeared in the 1960 patent shown in Figure 1.10. The system uses diodes to rectify the signal received by an antenna to generate DC power and drive a transistor oscillator to produce an identifying signal at a separate frequency $[1,11]$.

transmitted signal frequency $\mathrm{f} 1$

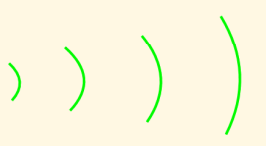

folded dipole

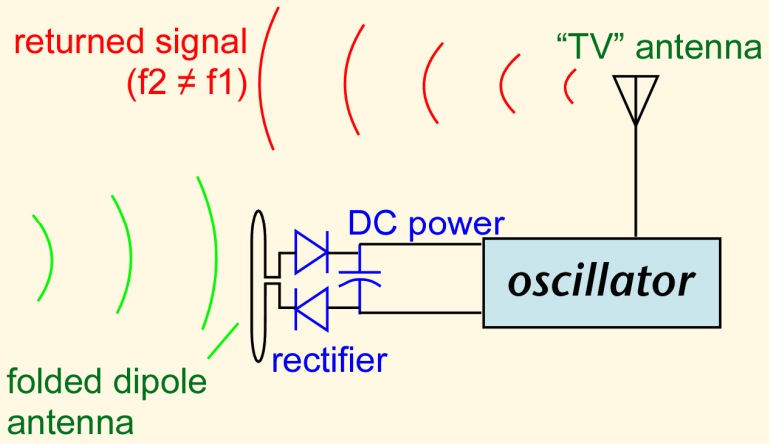

Figure 1.10: RFID Application; After Crump, US Patent 2,943,189, Filed 1956, Granted 1960 [11]

The first major commercial implementation of RFID technology (1970) used inductive coupling for tag-reader communications. Figure 1.11 shows an example of inductively coupled transponders. The reader detects voltage changes across the coil while sweeping frequency until tag resonance occurs. Inductive coupling requires close tag-reader proximity. To increase read range, a radiative coupling scheme, similar to the Crump patent [11], was further modified to support more complex circuitry for signal processing. For example, a more efficient AC-DC rectifying circuit was implemented to supply more driving power to the circuitry. 


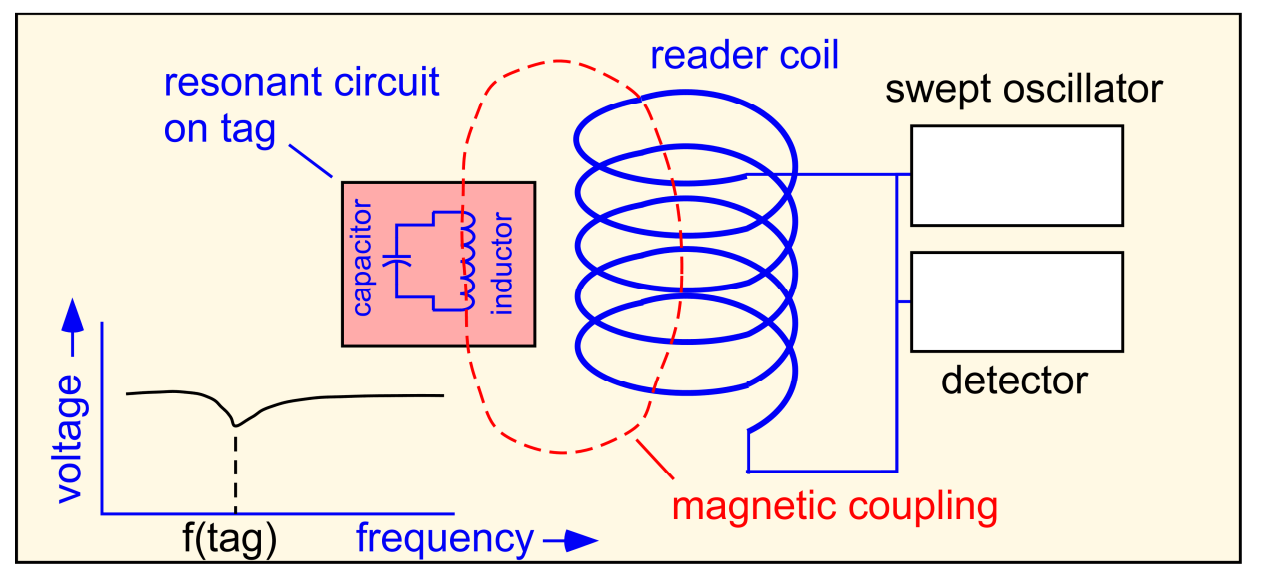

Figure 1.11: Inductively Coupled Identification System Example [1]

\subsubsection{UHF RFID Tag Systems}

A schematic depiction of a passive tag is illustrated in Figure 1.12. The tag antenna, often embedded in the tag, receives the reader signal and produces an RF voltage which is rectified and filtered by a diode and capacitor to produce DC voltage for logic circuitry and memory. Since the tag is usually powered off, passive tag memory must be non-volatile. Modern tag ICs typically requires $10-30 \mu \mathrm{W}(-20$ to $-15 \mathrm{dBm})$ for read operations. Accounting for the diode's turn-on voltage $(0.3 \mathrm{~V}$ to $0.7 \mathrm{~V})$, the rectification stage exhibits about $30 \%$ efficiency; therefore, the antenna is required to deliver $30-100 \mu \mathrm{W}$ (-15 to $-10 \mathrm{dBm}$ ) of power to the circuitry. Reader data is passed through a diode and capacitor stage (envelope detection) to accomplish reader information demodulation. Finally, the antenna impedance is varied by a field-effect transistor (FET) switch to modify the signal reflected back to the reader [1]. 


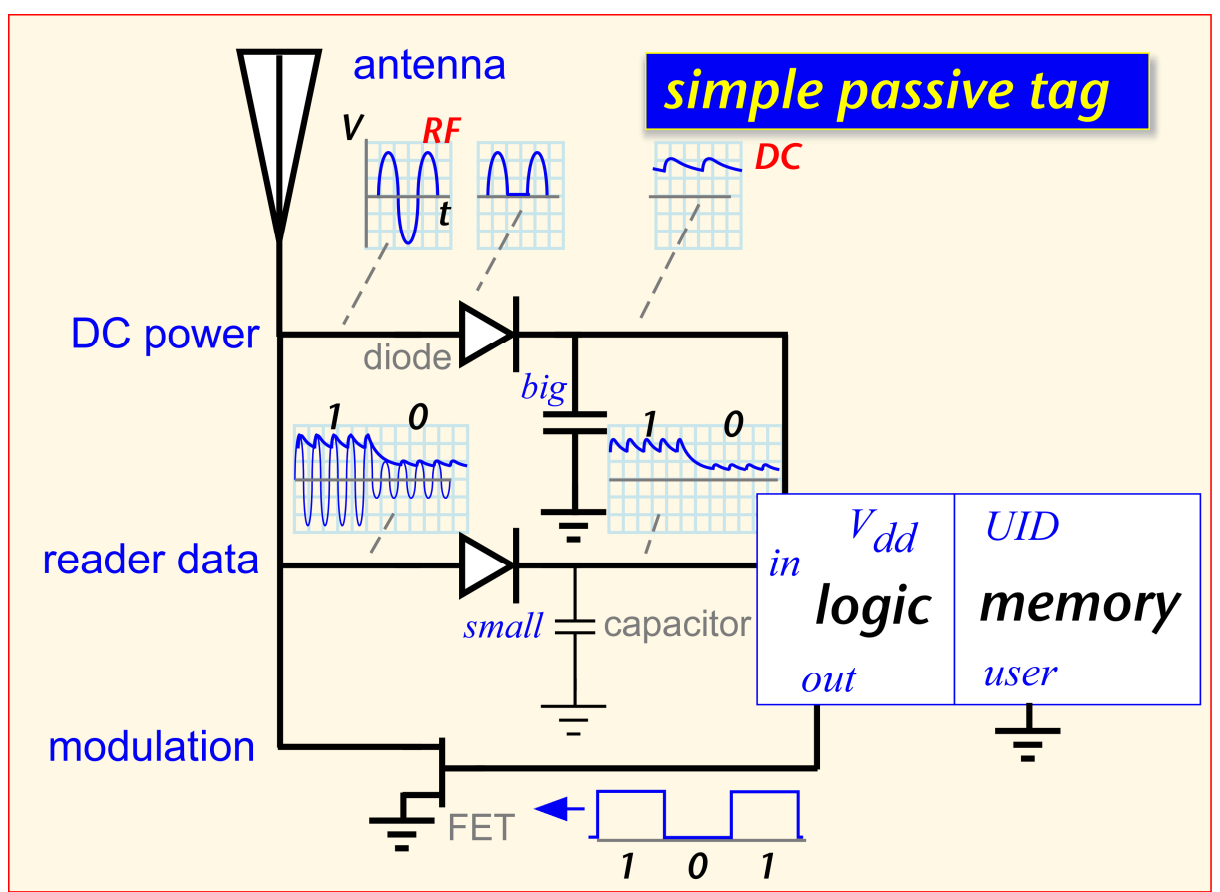

Figure 1.12: Passive RFID Tag Schematic [1]

Figure 1.13 (a) shows an example of a typical UHF RFID Tag. The antenna structure is deposited on a plastic (PET) substrate, known as an inlay. The RFID IC is mounted on a strap, a plastic substrate with attachment pads as shown in Figure 1.13 (b).

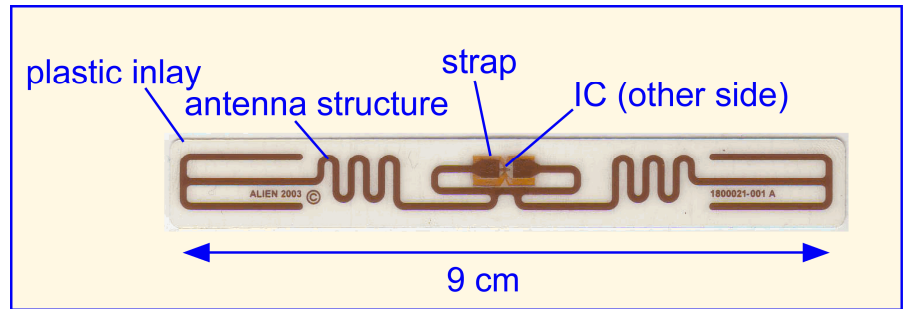

(a)

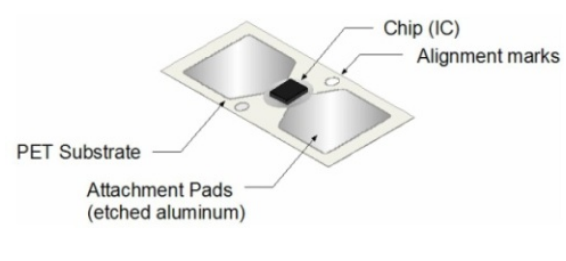

(b)

Figure 1.13: (a) Commercial Passive UHF Tag (Alien Technology Model 9238 'Squiggle') (b) Strap Package 
The strap package IC, Monza 3 model, used in this project was supplied by Sirit Inc, an RFID technology company. Table 1-2 provides Monza 3 specifications.

Table 1-2: RFID IC Specifications [12]

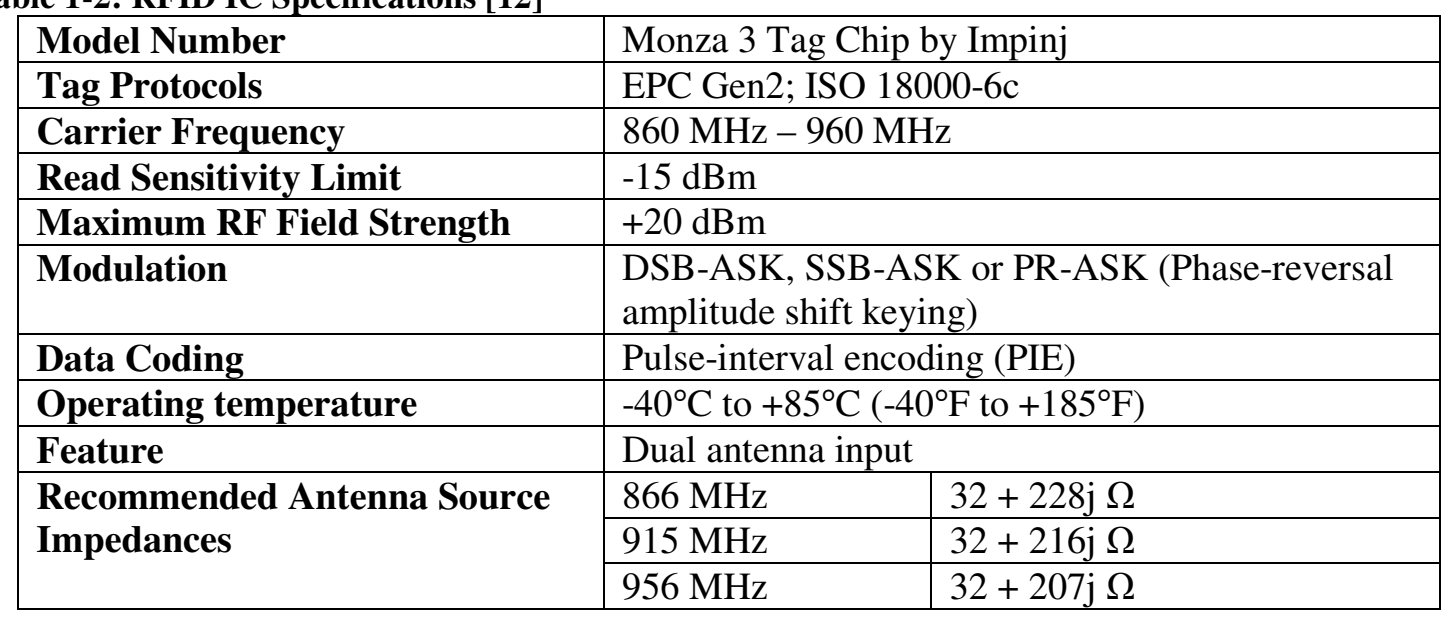

\subsection{Project Objectives and Organization}

RFID applications require small, low-cost RFID tags. IC cost is generally fixed by vendors; hence, RFID tag antenna material and fabrication cost must be minimized. The project objective is to design, fabricate and test low-cost UHF RFID tags. The conductive tag antenna area using additive patterning fabrication (adding conductive traces to the substrate) is minimized. This project established Cal Poly's first ever UHF RFID tag fabrication processes.

Chapter 2 covers UHF RFID tag fabrication, including antenna fabrication, IC-antenna attachment and inlay (plastic substrate with antenna and IC) post-processing. Chapter 3 describes UHF RFID design. To maximize read range, it is critical to receive and use RF power efficiently. As a result, tag antenna design plays an important role in tag performance. The key factor is to optimize impedance matching between the antenna and RFID IC to allow maximum power transfer at the desired frequency. UHF tag 
antenna design for optimized impedance matching is accomplished through electromagnetic (EM) simulation tool EMPro [47]. The project design minimizes antenna pattern silver ink requirements and simulation complexity by using only two structures: folded dipole with T-matching structure for impedance matching. Chapter 4 describes procedures to measure tag read range. 


\section{UHF RFID TAG FABRICATION}

\subsection{Fabrication Process Overview}

The final UHF RFID tag is a smart label with embedded inlays as shown in Figure 2.1(a). Figure 2.1(b) outlines the smart label manufacturing process. Individual ICs are separated and mounted on a strap package [Figure 1.13(b)] using high-precision attachment methods prior to inlay attachment. Low-precision strap-antenna attachments can be done by standard assembly techniques, such as the use of conductive adhesive.

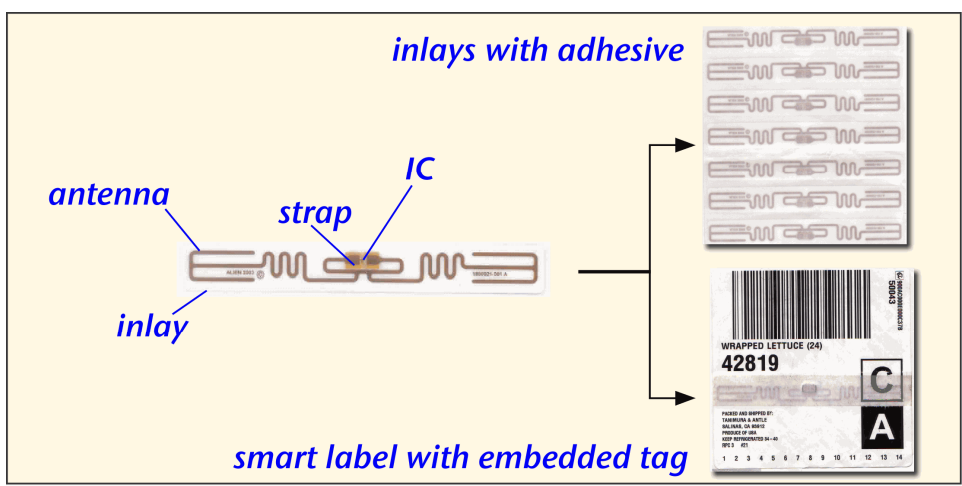

(a)

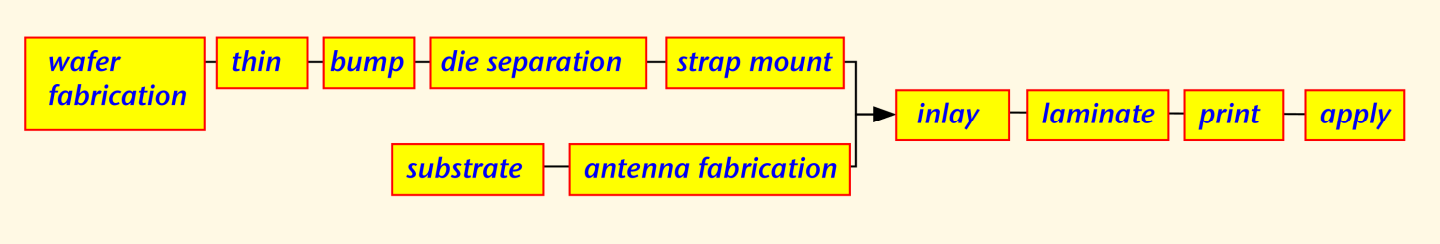

(b)

Figure 2.1: (a) Smart-Label Configurations and (b) Manufacturing Process [1]

Section 2.1.1 discusses the antenna fabrication process used in this project while Section 2.1.2 addresses the strap-to-antenna and IC-to-antenna fabrication processes. Finally, Section 2.1.3 addresses inlay processing following IC-antenna attachment. 


\subsubsection{Antenna Fabrication}

A common substrate material is polyethylene terephthalate (PET), an inexpensive and durable plastic with chemical resistance and low dielectric constant (3.0 to 3.9). PET film model Teijin ${ }^{\circledR}$ Tetoron ${ }^{\circledR}$ SL film, supplied by Sirit Inc, has low thermal shrinkage $\left(0.2 \%\right.$ size shrinkage at $150^{\circ} \mathrm{C}$ after 30 minutes $)$ and can withstand the heating process (10-20 minutes at $\left.130^{\circ} \mathrm{C}\right)$ during antenna fabrication [13].

Candidate conductive materials used for tag antenna traces include sheet copper and silver particle embedded polymer matrix conductive ink. Sheet resistance is the resistance of a thin square-shaped material volume with contacts at opposite sides of the square ( $\mathrm{m} \Omega /$ square). Silver ink datasheets typically specify sheet resistivity in $\Omega / \mathrm{sq} / \mathrm{mil}$ (ohms sheet resistance per square, 1mil thickness). Copper thickness of 10-40 microns $(\mu \mathrm{m})$ can be achieved, corresponding to sheet resistance values less than $1 \mathrm{~m} \Omega /$ square. On the other hand, silver particle embedded conductive inks generally exhibit sheet resistances of $12-20 \mathrm{~m} \Omega /$ square, about 10 times greater than solid silver or copper. A tag with a length to width (aspect) ratio of 20 has a DC resistance of approximately $0.4 \Omega$, negligible for most antenna designs [1].

Silver ink, XCSD-006N, is recommended by Sirit Inc and available through the Cal Poly Graphic Communication Department. The XCSD-006N silver ink has a resistivity less than $6 \mathrm{~m} \Omega / \mathrm{sq} / \mathrm{mil}$ and is designed for RFID printed antenna applications [14]. Silver ink commercial cost is about US\$1.0 per gram; approximately $1 \mathrm{mg}$ of silver ink can produce one tag antenna. 
Minimizing fabrication cost is an important factor for RFID applications. Cost reduction requires additive patterning techniques in which conductive traces are added to the substrate as opposed to removing (subtractive patterning). Tag antennas are also designed with minimized conductive areas to further limit cost. Appendix $\mathrm{C}$ summarizes antenna fabrication techniques along with benefits and drawbacks.

Initially, inkjet printing was considered but rejected due to printing machine purchase cost (US\$50,000 dollars: DMP-2831 Materials Inkjet Deposition system [48]). The screen-printing technique was eventually selected because it is an easy-to-use additive patterning technique that does not require expensive equipment or chemicals. Also, conductive ink screen-printing is available through the Cal Poly Graphic Communication Department.

Figure 2.2 illustrates the screen printing technique. Ink is placed on an inkblocking stencil that includes open mesh areas (logos). The semi-permeable barrier is composed of interconnected plastic strands. By sweeping a roller or squeegee across the screen stencil, ink is pressed through the mesh to produce sharp-edged images on the substrate.

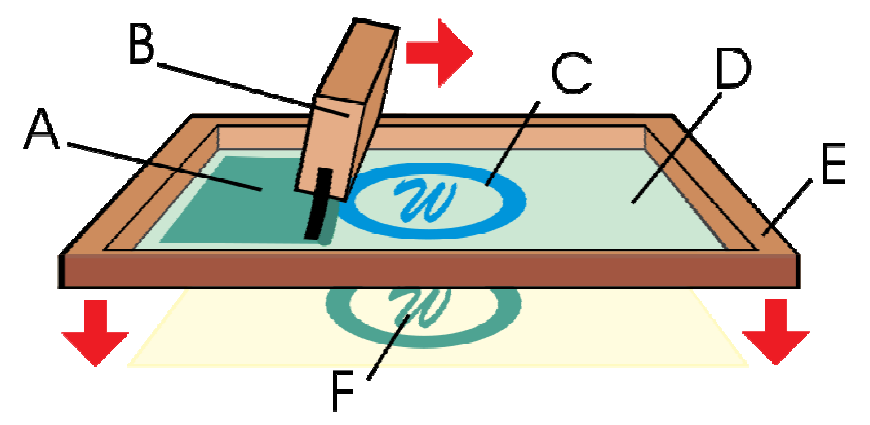

Figure 2.2: Screen Printing, A: Ink, B: Roller or Squeegee, C: Open Area, D: Ink-Blocking Stencil, E: Mesh Polyester Screen, F: Substrate 
The first step in the printing process is to create the screen (produced by the Graphic Communication Department). The antenna pattern is exported from an EM simulation software EMPro to an image file format (.dwf) using the procedure described in Appendix D. This image file is used to create a positive photoresist printout as shown in Figure 2.3. Through chemical etching, the printout generates the ink-blocking stencil, red sections in Figure 2.4.
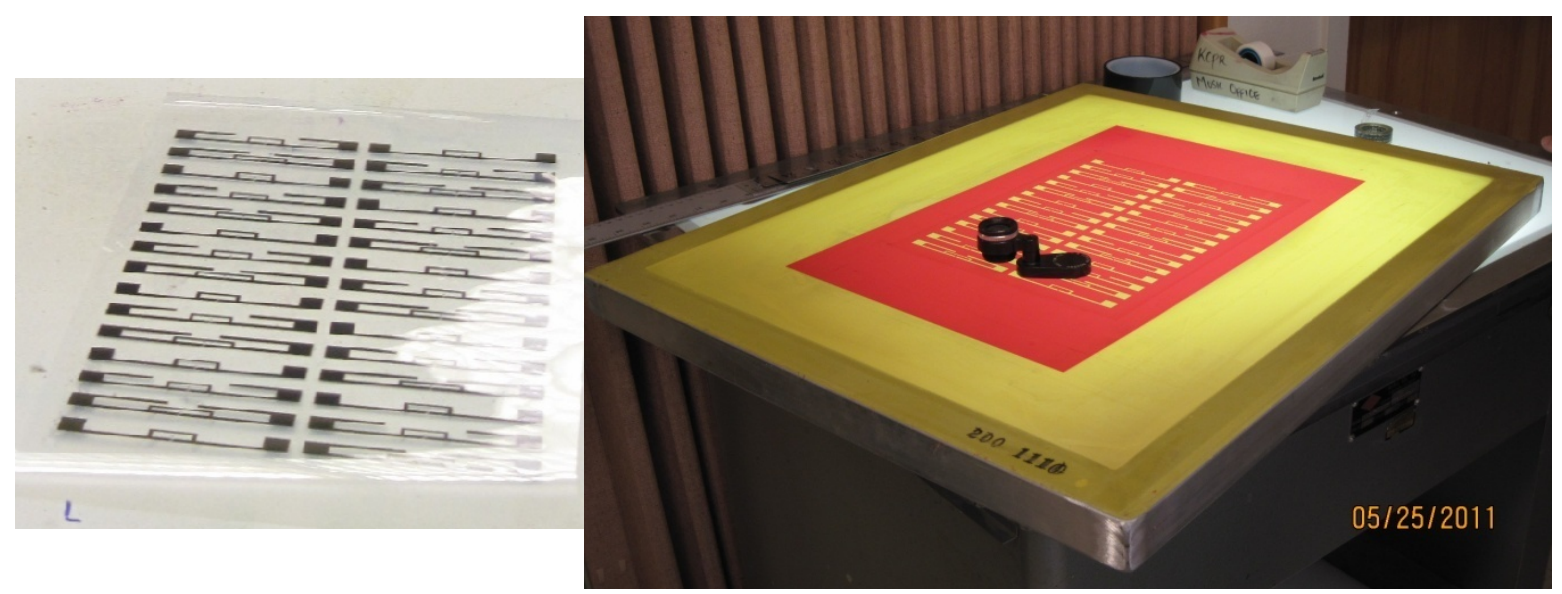

Figure 2.3: RFID Antenna Positive Photoresist Pattern

Figure 2.4: RFID Antenna Print Screen

Figure 2.5 shows the screen printing machine. The PET substrate is placed on the table below the screen, "Substrate Table". Electric Static on the PET film is removed by brushing with a plastic rod before the screen is lowered onto the substate. A gap between the substrate and screen allows separation following roller application. The screen is designed to hold the ink during and after substrate-screen separation to preserve the conductive ink pattern. 


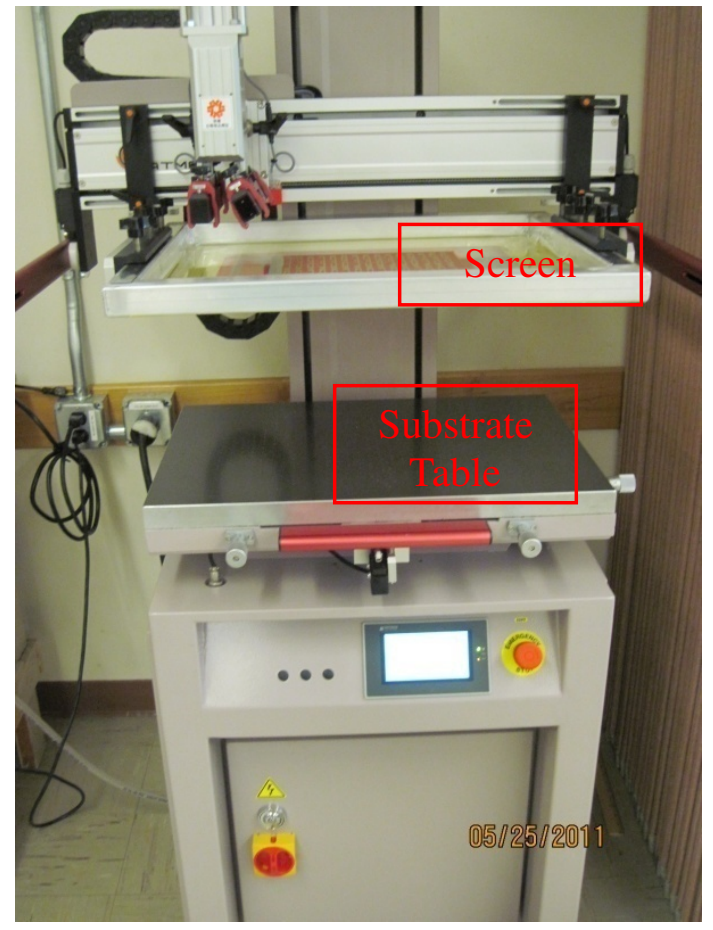

Figure 2.5: Screen Printing Machine

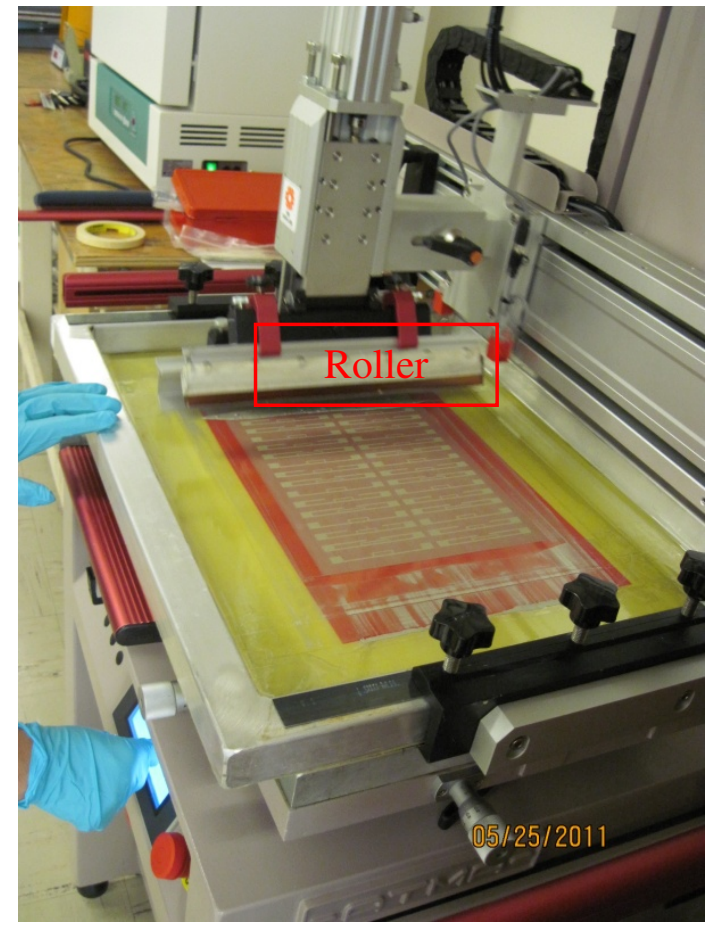

Figure 2.6: Roller Over Stencil Pattern

Ink is placed on the left side of the screen and the roller is applied from left to right to press ink into the open areas. A second roller application from right to left completes the pattern.

Figure 2.7 shows a picture of a substrate sheet with printed antenna patterns for multiple tags. The printed antenna is then heat-cured in a $130^{\circ} \mathrm{C}$ oven for 15 minutes to realign the silver ink's molecular structure (improve conductivity). The antenna is now ready for IC attachment. 


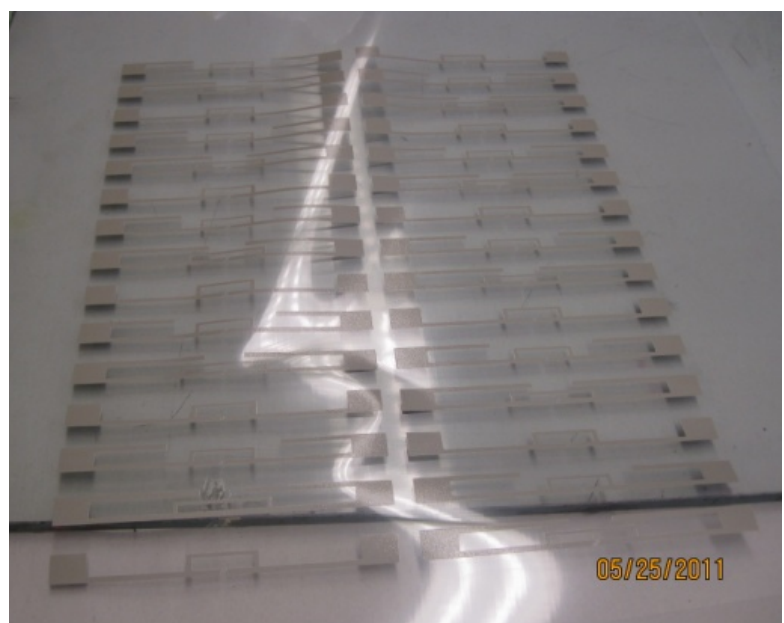

Figure 2.7: Printed RFID Tag Antenna

\subsubsection{IC-Antenna Attachment Procedures}

Historically, IC die connections to IC package pads are accomplished through wire bonding. Figure 2.8 (a) illustrates typical IC package wire bonding techniques. A thin (50 to 100 micron diameter) aluminum or gold wire connects the IC's conductive pad (using ultrasonic scraping and/or heating) to the package's conductive pad. To use this process in the IC-antenna connection, the IC must be held in place by an adhesive on the face-up antenna with the IC also face-up. The IC's conductive pads are then wire bonded to the antenna pads. Wire bonding produces highly reliable and low-inductance connections. However, this process requires precise chip placement and wire-bonding equipment; hence, is relatively expensive [15].

Another mature IC assembly process is the flip chip in which metal extrusions are formed on the chip's contact pad surface. The chip is inverted and placed over strap or tag antenna conductive pads (possibly covered with solder for alignment). Heat is applied to melt the solder and surface tension aligns the contact pads. It is relatively expensive and awkward to create suitable solder pads on typical RFID substrates [15]. 


\section{WIRE BOND}

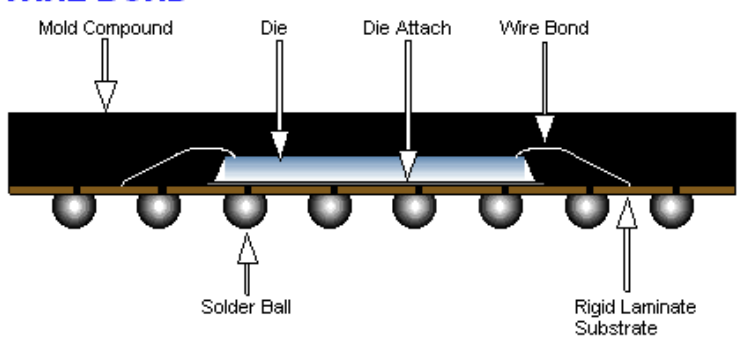

(a)

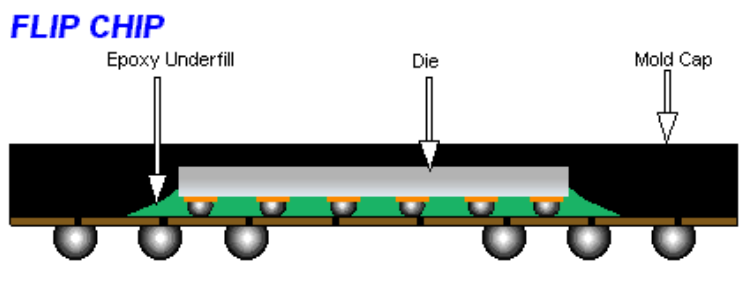

(b)

Figure 2.8: IC-Antenna Attachment Techniques (a) Wire Bonding, (b) Flip Chip

A less expensive approach uses anisotropic (conductive in one direction) conductive adhesives. Figure 2.9 (a) demonstrates adhesive application between the antenna and bare die or IC strap. As illustrated in Figure 2.9 (b), conductive adhesives contain low-concentration dispersions of conductive particles (10\% - 50\% of volume) [16]. Although the adhesives are substantially insulating under normal conditions, when chip metal contacts are pressed onto the substrate metal contact under pressure and heat, electrical connections are achieved.

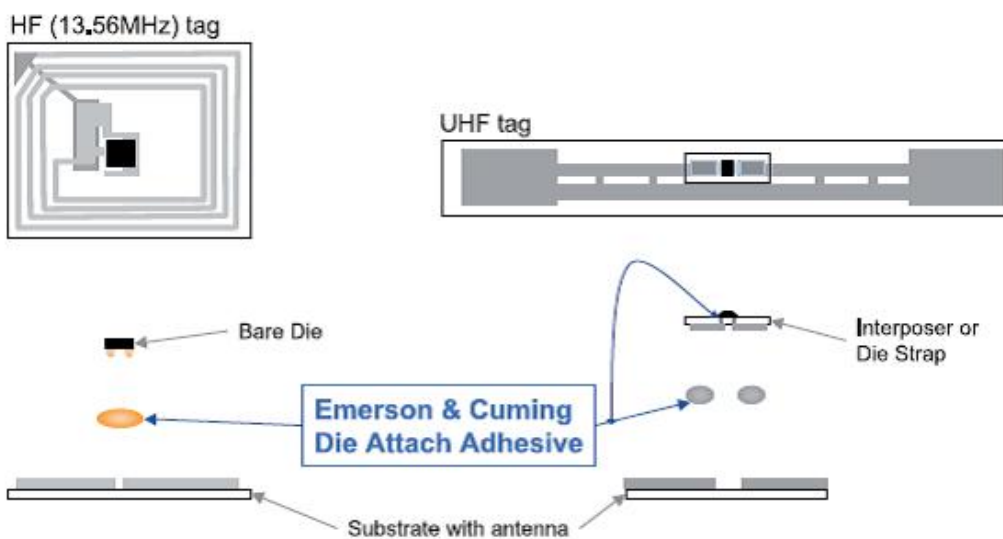

(a)

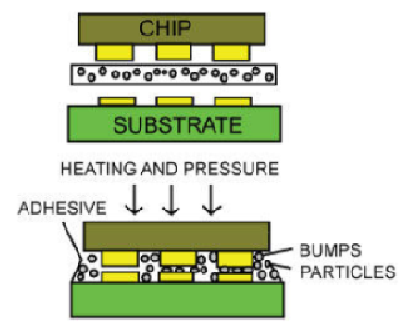

(b)

Figure 2.9: IC-Antenna Attachment using Anisotropic Conductive Adhesives [17, 18]

The last approach, employed exclusively by high volume manufacturers, uses the strap package, an IC attached to substrate conductive pads using flip-chip assembly. The IC is attached to the antenna using anisotropic conductive adhesives. This approach is 
particularly suitable in a laboratory environment because the strap-antenna attachment can be done manually using conductive adhesive. Other strap-antenna attachment methods include heat curing epoxy, UV curing epoxy and ultrasonic welding (using local high-frequency ultrasonic acoustic vibrations to create solid-state weld). The conductive adhesive approach was used for this project as demonstrated in Figure 2.10. A conductive double-sided tape (EL-9032, Adhesive Research, Inc.) was used. The conductive tape can be applied at room temperature $\left(>10^{\circ} \mathrm{C}\right.$ or $\left.50^{\circ} \mathrm{F}\right)$. The maximum operating temperature is $121^{\circ} \mathrm{C}\left(250^{\circ} \mathrm{F}\right)$. The figure shows conductive tape application to the antenna trace.

Aligning the strap package to the printed antenna registration dot, the strap is held firmly and attached to the substrate.

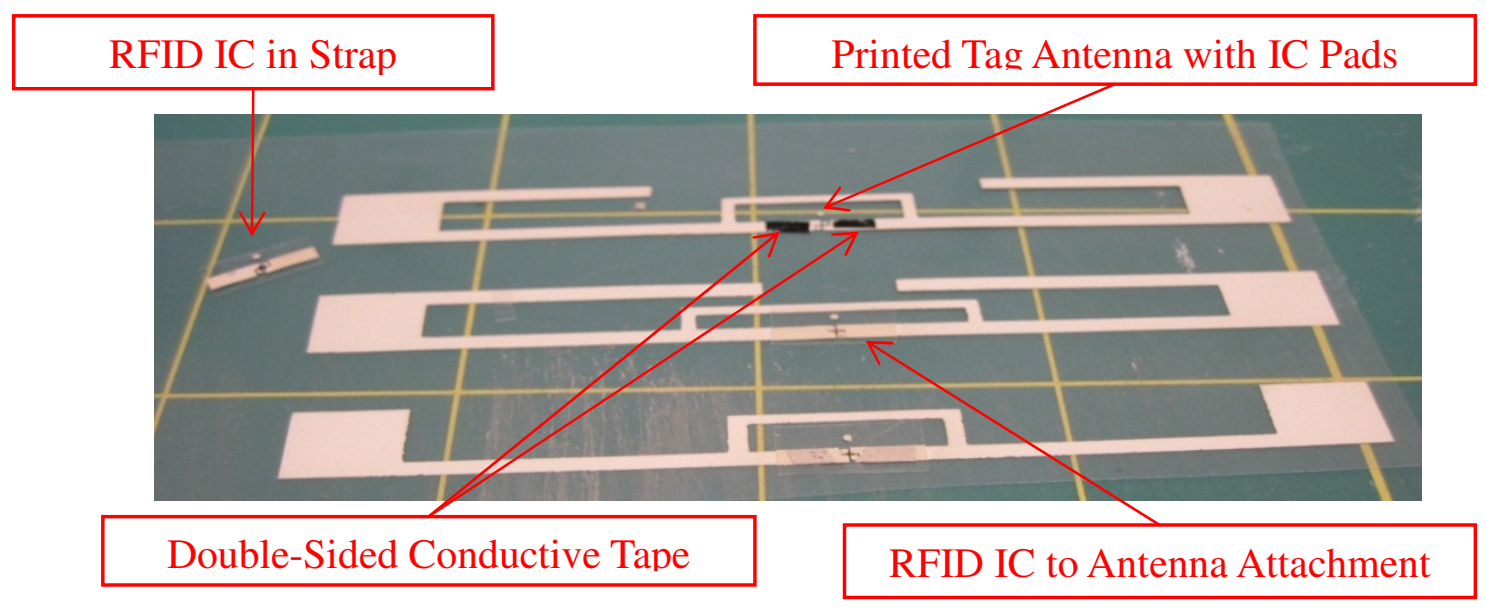

Figure 2.10: IC-Antenna Attachment Using Strap Package and Conductive Tape

A new strap assembly method, Fluidic Self-Assembly (FSA), was developed by Alien Technolgy for strap package mass-production, see Figure 2.11. This method can be applied in parallel. ICs are suspended in a fluid and circulated within a system. The substrate contains IC conforming depressions. An upper silver ink layer and conductive 
vias, (the strap superstrate) are used to connect ICs to the substrate. The superstrate and substrate layers are laminated together, fully encapsulating the IC [15] for protection against the external environment. Figure 2.11shows a cross-sectional view of an FSAassembled strap.

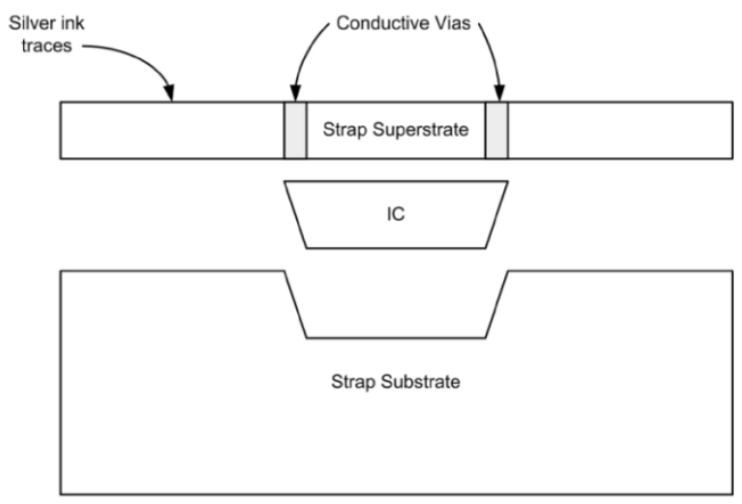

Figure 2.11: Fluidic Self-Assembly (FSA)-Assembled Strap Cross-Section [15]

\subsubsection{Inlay Post-Processing}

Following IC attachment, polymer coatings may be applied to protect the IC and antenna. If the inlays are used independently, they are laminated onto a paper backing. Inlays can also be laminated on a plastic adhesive-backed label and passed through a smart label printer.

In this thesis, a typical desktop laminating machine is used for tag lamination. This method does not degrade tag read range performance (see Section 4.2). 


\section{UHF RFID ANTENNA DESIGN}

\subsection{Design Process Overview}

Figure 3.1 is the UHF RFID antenna design process flow graph [19]. The following subsections describe each block in the flow graph.

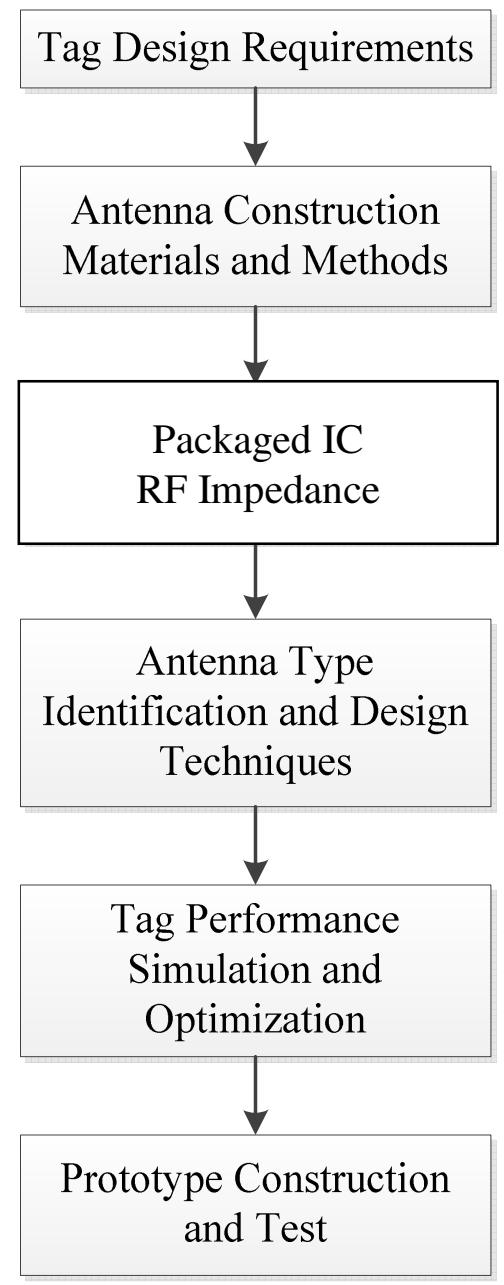

Figure 3.1: RFID Tag Antenna Design Flow Graph

\subsubsection{Tag Design Requirements}

Read range is defined by the RFID IC's read sensitivity; the minimum threshold power for normal IC operation). 
The Friis free-space formula is used to determine the received power [20]

$$
P_{r}=P_{t} \frac{G_{t} G_{r} \lambda^{2}}{(4 \pi R)^{2}}
$$

$\lambda$ : wavelength

$\mathrm{P}_{\mathrm{t}}:$ transmit power

$\mathrm{G}_{\mathrm{t}}:$ transmit (reader) antenna gain

$\mathrm{G}_{\mathrm{r}}$ : receive (RFID tag) antenna gain

$\mathrm{P}_{\mathrm{r}}$ : received power

$\mathrm{R}$ : distance between transmit and receive antenna

For the reader-tag system, (3.2) becomes

$$
P_{\text {tag }}=P_{\text {reader }} \frac{G_{\text {reader }} G_{t a g} \lambda^{2}}{(4 \pi R)^{2}}
$$

The power transmission coefficient, $\tau$, quantifies impedance mismatch between the reader antenna and RFID tag. The power available to the IC is expressed as,

$$
P_{I C}=\tau P_{t a g}
$$

in which [19],

$$
\tau=\frac{4 R_{c} R_{a}}{\left|Z_{c}+Z_{a}\right|^{2}}, \quad 0 \leq \tau \leq 1
$$

$Z_{c}$ : chip impedance $\left(Z_{c}=R_{c}+X_{c}\right), R_{c}$ is chip resistance, $X_{c}$ is chip reactance 
$Z_{a}$ : antenna impedance $\left(Z_{a}=R_{a}+X_{a}\right), R_{a}$ is antenna resistance, $X_{a}$ is antenna reactance

$\tau$ is the ratio of actual IC available power to the maximum available power.

$$
P_{I C}=\tau P_{\text {tag }}=\tau P_{\text {reader }} \frac{G_{\text {reader }} G_{\text {tag }} \lambda^{2}}{(4 \pi R)^{2}}
$$

If the power available to the IC ( $\left.\mathrm{P}_{\mathrm{IC}}\right)$ is the minimum threshold power necessary to power the RFID IC, $\mathrm{P}_{\text {th }}$, distance $\mathrm{R}$ in equation 3.5 is the read range $\mathrm{r}$ (according to the read range definition).

$$
P_{t h}=\tau P_{\text {tag }}=\tau P_{\text {reader }} \frac{G_{\text {reader }} G_{\text {tag }} \lambda^{2}}{(4 \pi r)^{2}}
$$

Solving for $\mathrm{r}$,

$$
r=\frac{\lambda}{4 \pi} \sqrt{\frac{P_{\text {reader }} G_{\text {reader }} G_{\text {tag }} \tau}{P_{t h}}}
$$

In the United States, the Federal Communications Commission (FCC) allows unlicensed use in the 902-928 MHz band under the following constraints. The maximum reader transmit power cannot exceed $1 \mathrm{~W}$ (or $30 \mathrm{dBm}$ ) and EIRP (Equivalent Isotropically Radiated Power) cannot exceed 4W (36dBm). The RFID reader EIRP is the product of the reader antenna transmit power and gain. The IC's read sensitivity specification, $\mathrm{P}_{\text {th }}$, typically ranges from $-15 \mathrm{dBm}$ to $-10 \mathrm{dBm}$. 
Bandwidth defines the frequency range over which the tag's impedance matching meets the $\left|S_{11}\right|<-10 \mathrm{~dB}$ requirement. $S_{11}$ is the complex reflection coefficient defined by

$$
S_{11}=\frac{Z_{\text {in }}-Z_{0}}{Z_{\text {in }}+Z_{0}}
$$

$\mathrm{Z}_{0}$ : transmission line intrinsic impedance

Since each country defines UHF RFID operating frequency bands, worldwide tag operation requires a bandwidth of $100 \mathrm{MHz}$ or $11 \%$ around $900 \mathrm{MHz}$ center frequency[19].

This thesis focuses on impedance matching the antenna to the IC. The IC impedance is generally complex and capacitive. For maximum power transfer between the IC and antenna (maximum read range), the antenna impedance must approximate the IC impedance's complex conjugate. Figure 3.2 illustrates the relationship between the antenna $\left(\mathrm{R}_{\mathrm{a}}+\mathrm{j} \mathrm{X}_{\mathrm{a}}\right)$ and IC impedance $\left(\mathrm{R}_{\mathrm{c}}+\mathrm{j} \mathrm{X}_{\mathrm{c}}\right)$ and read range. The figure depicts IC impedance variations with respect to frequency and complex conjugate match.

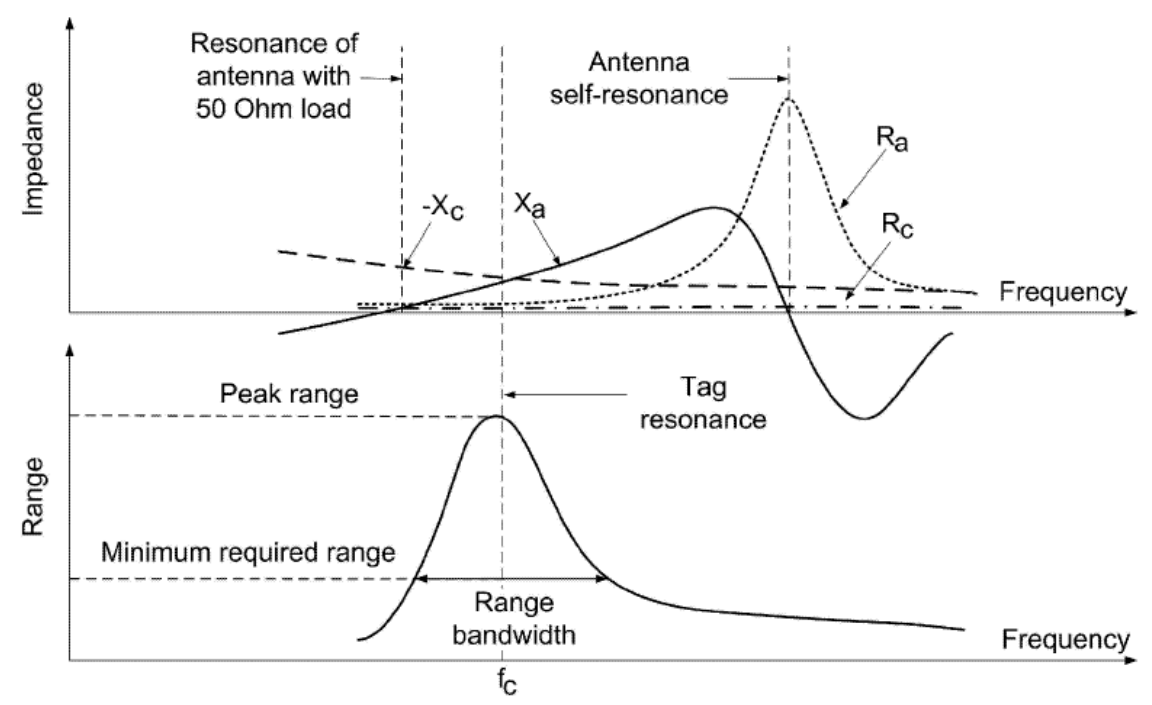

Figure 3.2: Antenna Impedance and Chip Impedance vs. Frequency for a Typical RFID Tag [19] 


\subsubsection{Antenna Construction Materials and Methods}

The strap package IC is bonded to the antenna manually using conductive tape.

The detailed fabrication process is provided in Chapter 2. Table 3-1 lists UHF RFID tag fabrication materials.

Table 3-1: UHF RFID Tag Fabrication Materials

\begin{tabular}{|l|l|}
\hline Description & P/N or Model Number \\
\hline RFID Tag IC & Impinj Monza 3 [12] \\
\hline RFID Tag Substrate (PET) & Teijin Tetoron SL film [13] \\
\hline Conductive Silver Ink & Spraylat XCSD-006 Polymer Thick Film \\
Conductive Tape (IC-Antenna & EL-9032 Conductive Adhesive by Adhesive \\
Attachment) & Research Inc [21] \\
\hline
\end{tabular}

\subsubsection{Packaged ASIC RF Impedance Measurement}

The RFID tag antenna is designed specifically for the available RFID IC (Monza 3). Due to IC dimensions, a specialized RF wafer probe is necessary for accurate impedance measurements using a Vector Network Analyzer (VNA). A transistor test fixture (Inter-Continental Microwave TF-30001-B) was used to acquire approximate impedance measurements for the Monza 3 strap package IC. Using a calibration kit (Inter-Continental Microwave TRL-3004), the test fixture is calibrated to the strap's conductive trace with $-15 \mathrm{dBm}$ power level (minimum IC operating power). Figure 3.3 shows the test setup. 


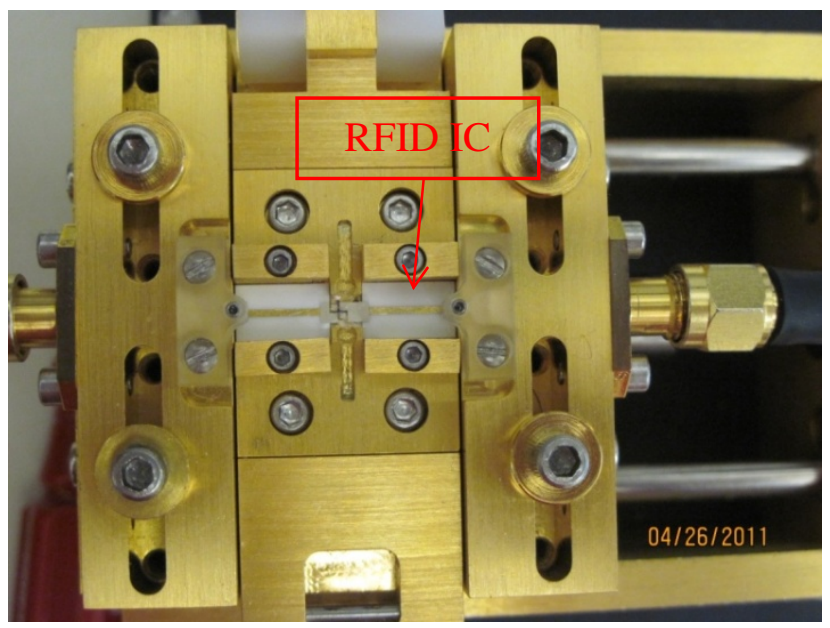

Figure 3.3: RFID IC (Strap) on ICM TF-30001-B Test Fixture

Two RFID ICs were measured and the resulting Smith Charts are captured in Figure 3.4 and Figure 3.5. Complex impedance is plotted against frequency in Figure 3.6 and the results are summarized in Table 3-2 for datasheet comparisons. The measured impedance values (both real and imaginary components) vary within a $100 \Omega$ span and do not match the datasheet with about $400 \Omega$ difference in reactance (see Table 3-2). The additional conductive trace on the strap causes deviations in reactance. However, the measurement did show the capacitive nature of the IC's impedance.

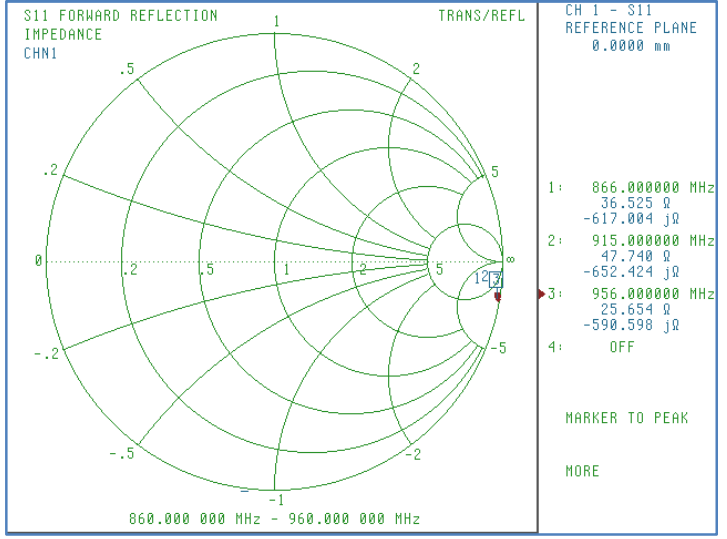

Figure 3.4: RFID IC \#1 Impedance Measurement

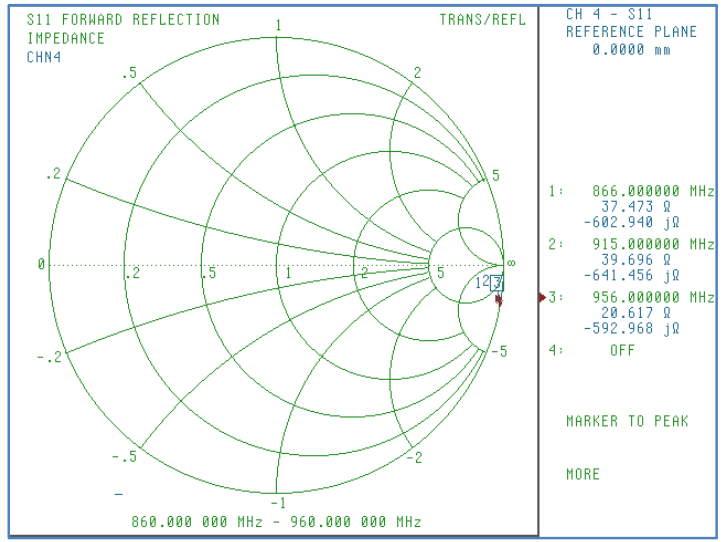

Figure 3.5: RFID IC \#2 Impedance Measurement 


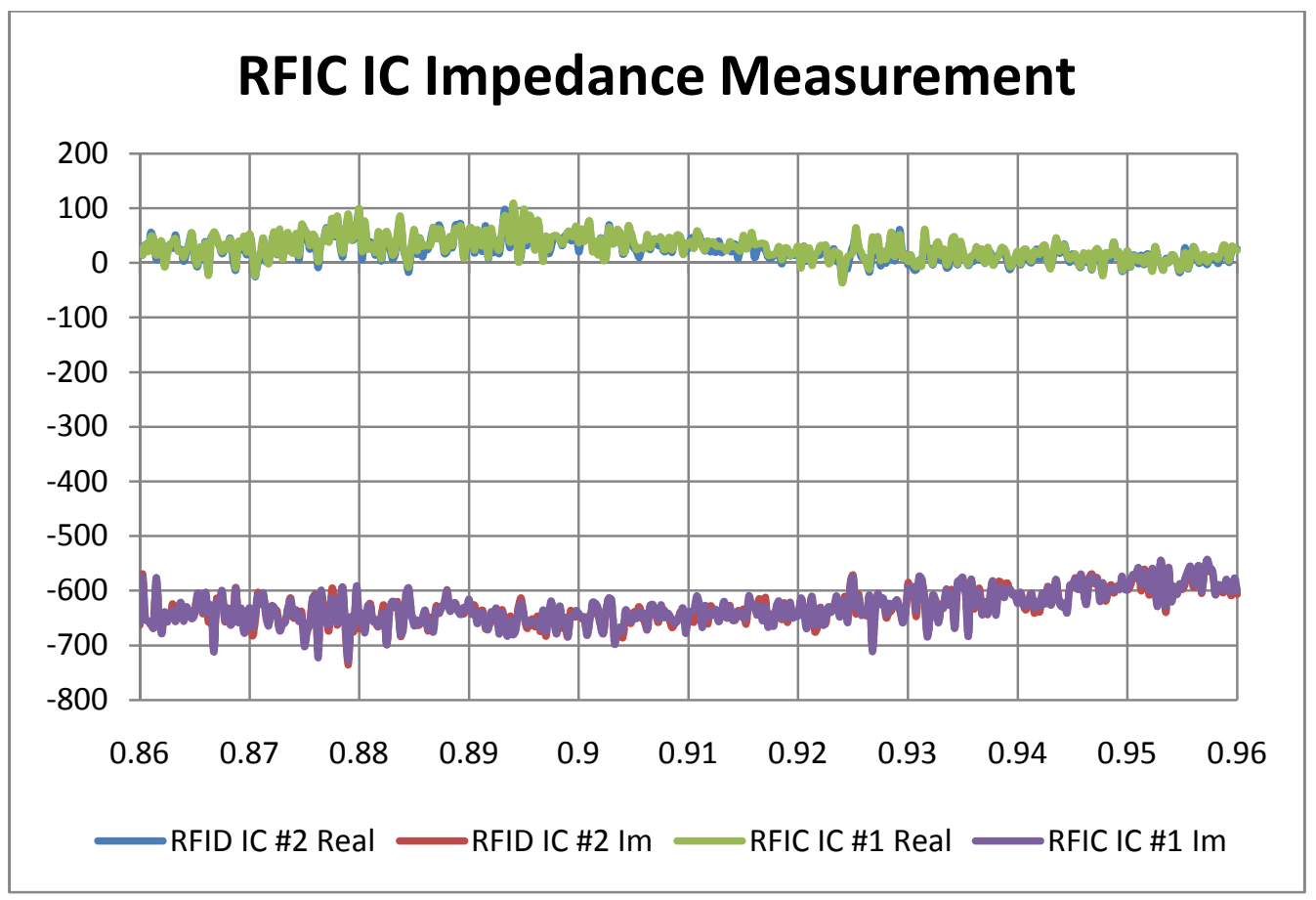

Figure 3.6: RFIC Measured Impedance vs. Frequency

Table 3-2: RFID IC Impedance Measurement

\begin{tabular}{|c|c|c|c|}
\hline & Datasheet Spec & Measured \#1 & Measured \#2 \\
\hline $\mathbf{8 6 6} \mathbf{~ M H z}$ & $32-\mathrm{j} 228 \Omega$ & $36.5-\mathrm{j} 617 \Omega$ & $37.5-\mathrm{j} 603 \Omega$ \\
\hline $\mathbf{9 1 5} \mathbf{~ M H z}$ & $32-\mathrm{j} 216 \Omega$ & $47.7-\mathrm{j} 652 \Omega$ & $39.7-\mathrm{j} 642 \Omega$ \\
\hline $\mathbf{9 5 6} \mathbf{~ M H z}$ & $32-\mathrm{j} 207 \Omega$ & $25.6-\mathrm{j} 590 \Omega$ & $20.6-\mathrm{j} 593 \Omega$ \\
\hline
\end{tabular}

The Monza 3 IC measured impedance is $15-\mathrm{j} 180 \Omega$ [22] at $912 \mathrm{MHz}$ which differs from datasheet specifications. To determine the expected IC impedance, the Sirit tag antenna CAD drawing [23] was directly imported into the simulation tool. The extracted impedance serves as the reference impedance for project tag antenna designs. Figure 3.7 shows the tag model imported into EMPro. Section 3.1.5.3 illustrates simulation results. 


\section{भुचس}

Figure 3.7: EMPro Model, Sirit RSI-674 Inlay RFID Tag

\subsubsection{Antenna Type Identification and Design Techniques}

UHF RFID tags operate in the $902-928 \mathrm{MHz}$ band; the wavelength is $32.8 \mathrm{~cm}$ at $915 \mathrm{MHz}$ with a resonant dipole length of $16.4 \mathrm{~cm}$. Most adhesive shipping labels have maximum length constraints of $10 \mathrm{~cm}$; hence, a resonant dipole cannot be used directly. Secondly, the antenna must tolerate dielectric variations, constant rapid movement in the surrounding environment, and polarization variation. The required operating bandwidth is approximately $100 \mathrm{MHz}$. Finally, the antenna must drive a substantially capacitive (-100 to $300 \Omega$ reactance) load (RFID IC) [15].

A majority of commercial UHF RFID tag antennas employ a dipole antenna structure because of its isotropic radiation pattern (H-plane). Due to limited label size, a printed full-length dipole with resonant length is rarely used. Figure 3.8 shows the Sirit RSI-674 UHF RFID tag with highlighted design techniques. A detailed description is provided in the following section.

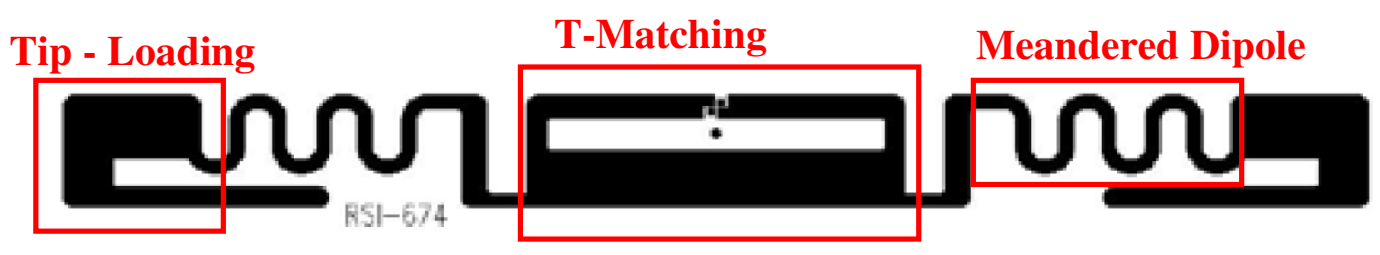

Figure 3.8: Sirit RSI-674 UHF RFID Tag Design Techniques 


\subsubsection{Tag Size Reduction Techniques}

Meandered, tip-loaded and fat dipoles are three common approaches for size reduction. Figure 3.9 shows a meandered dipole. Resonance is achieved with reduced projected length. Tag antenna bandwidth is directly proportional to the power dissipated and inversely proportional to the energy stored [15]; hence, the meandered dipole has narrower bandwidth due to reduced power dissipation caused by reduced radiation resistance (currents on sections normal to straight dipole sections cancel).

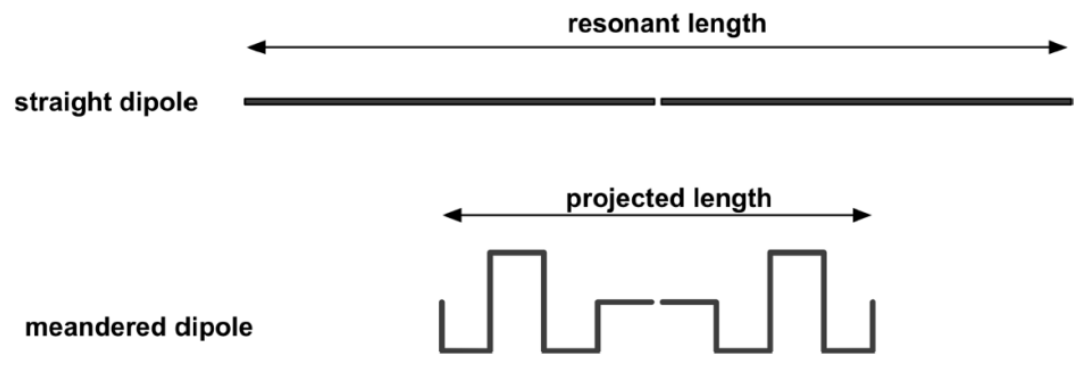

Figure 3.9: Meandered Dipole with Reduced Projected Length [15]

Tip-loaded dipoles (Figure 3.10) also reduce the projected length. Tip-loading is accomplished by increasing the structure width at the ends. Capacitive reactance decreases inverse relation between antenna capacitance and reactance while increasing the radiation resistance. The tip-loaded structure stores charge and creates a nearlyuniform current amplitude along the dipole. 


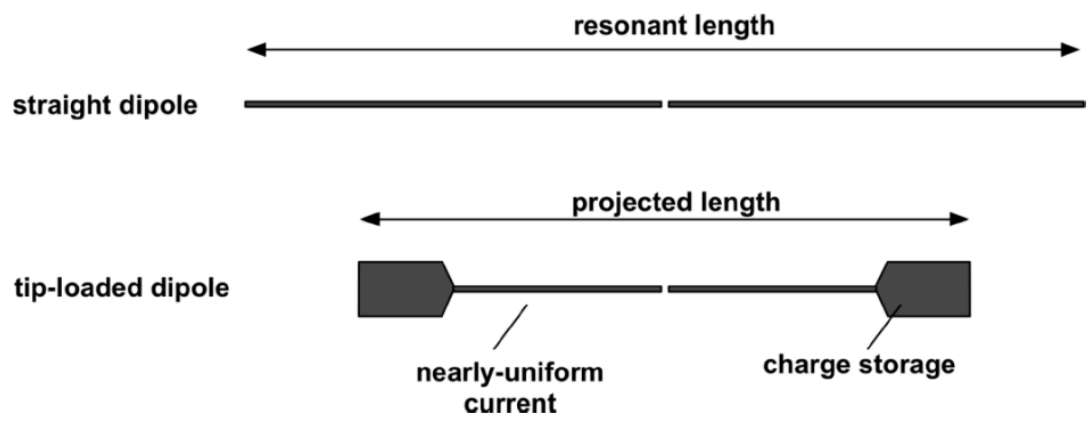

Figure 3.10: Tip-loaded Dipole with Reduced Projected Length [15]

Another technique for reducing dipole reactance (energy stored) and increase bandwidth is to use a fat dipole (Figure 3.11) [15]. Increased linewidth reduces resonant length. For example, a wire length to diameter ratio of 50 requires a resonant length of $0.475 \lambda$ compared to $0.500 \lambda$ (half-wave). For a ratio of 10 , the resonant length decreases to $0.455 \lambda$ [20]. The relationship between cylindrical dipole radius (a) and metallic strip width (w) for equivalent performance is $\mathrm{a}=0.25 \mathrm{w}$ [24]. The drawback for this approach is the increased conductive area which increases cost.

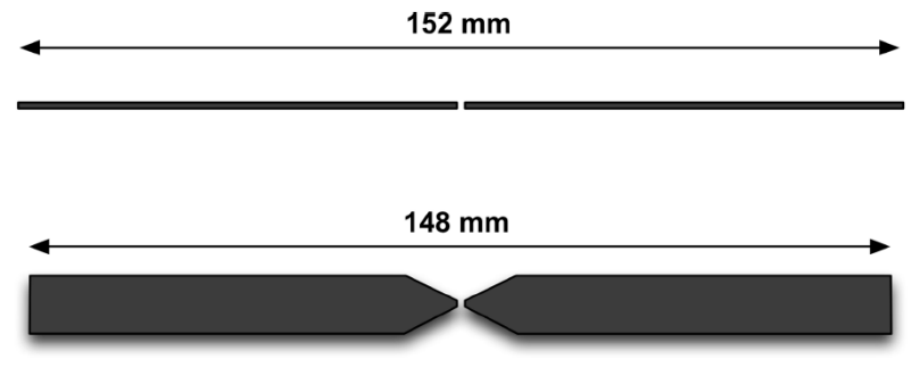

Figure 3.11: Fat Dipole with Reduced Projected Length [15]

\subsubsection{Impedance Matching Techniques}

Three types of impedance matching structures - inductively coupled loop, loading bar and modified T-matching - are shown in Figure 3.12. The inductively coupled loop 
adds equivalent inductance to the antenna. Impedance is affected by the distance between the loop and radiating structure, as well as loop shape $[25,26]$. The loading bar acts as a shunt component adjusting the antenna impedance to match with IC's. See Ref $[1,25]$ for more detailed descriptions for the two techniques.

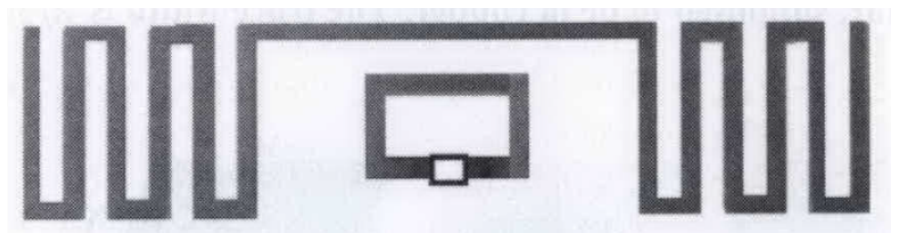

(a)

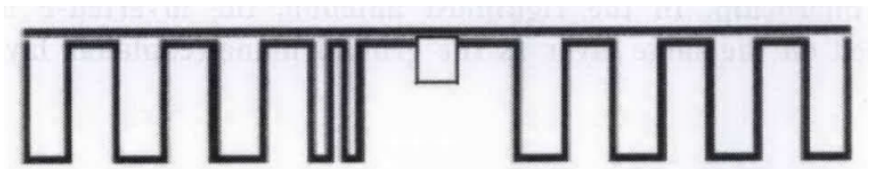

(b)

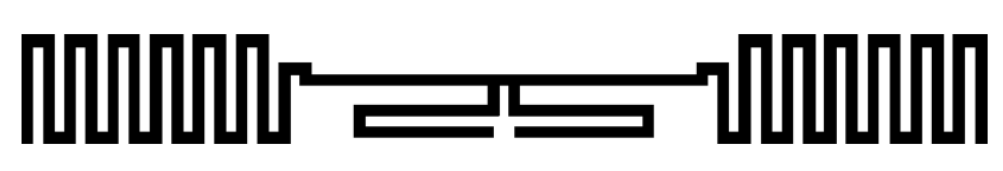

(c)

Figure 3.12: Meander Dipole Impedance Matching Structures (a) Inductively Coupled Loop [25], (b) Loading Bar [25], (c) T-Matching Structure [15]

In the T-matching structure, a second dipole is connected to the main dipole, Figure 3.13(a). Several authors have developed analytical/equivalent circuit models for the Tmatching structure $[22,25,27-28]$. The T-matching analytical model is based on the Uda model [29]. Figure 3.13 (b) illustrates the equivalent circuit model; variables are defined below: 
$\mathrm{Z}_{\mathrm{T}}$ : shorted-circuit stub input impedance formed by T-matching conductors and the dipole.

$\mathrm{Z}_{\mathrm{D}}$ : center fed dipole impedance without $\mathrm{T}$-matching

$\mathrm{Z}_{\text {Chip }}$ IC chip impedance

$\mathrm{k}$ : wave number, $2 \pi / \lambda$

$\mathrm{Z}_{\mathrm{ANT}}$ or $\mathrm{Z}_{\mathrm{Tag}}$ : antenna impedance at IC location

$\alpha$ : current splitting factor (defined in equation 3.10)

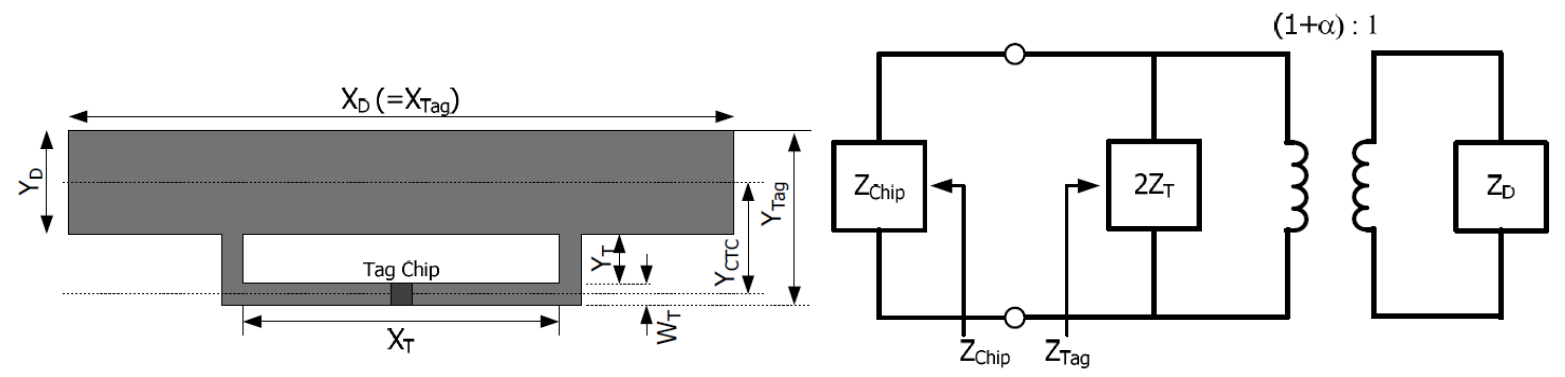

(a)

(b)

Figure 3.13: (a) Dipole with T-Matching Structure, (b) Equivalent Circuit [22]

The antenna impedance is modeled as [30]

$$
Z_{\text {Ant }}=Z_{\text {Chip }}^{*}=\frac{2 Z_{T}\left[(1+\alpha)^{2} Z_{D}\right]}{2 Z_{T}+(1+\alpha)^{2} Z_{D}}
$$

where,

$$
\begin{gathered}
\alpha=\frac{\log _{e}\left(\frac{Y_{C T C}}{W_{T}^{\prime}}\right)}{\log _{e}\left(\frac{Y_{C T C}}{Y_{D}^{\prime}}\right)}, \text { where } W_{T}^{\prime}=0.25 W_{T} \text { and } Y_{D}^{\prime}=0.25 Y_{D} \\
Z_{T}=j Z_{0} \tan \left(\frac{K X_{L}}{2}\right), \text { where } Z_{0}=276 \log _{10}\left(\frac{Y_{C T C}}{\sqrt{W_{L}^{\prime} Y_{D}^{\prime}}}\right)
\end{gathered}
$$


A T-matching structure matching chart for $\mathrm{X}_{\mathrm{D}}=0.5 \lambda, \mathrm{Y}_{\mathrm{D}}=0.01 \lambda, \mathrm{W}_{\mathrm{T}}=\mathrm{Y}_{\mathrm{D}} / 3$ and $\mathrm{Z}_{\mathrm{D}}=75 \Omega$ has been developed [25] [Figure 3.14(a)]. A similar chart [Figure 3.14(b)] was reproduced according to the analytic model, equation (3.9-3.11); however, the resulting chart differs with [25]. Uda Model limitations for T-matching antennas are examined in [28]; the authors suggest that the gap (IC location) was not included in the original folded dipole Uda model. Differences were found between T-matching EMPro simulation and analytical data, equation (3.9-3.11). Because of the unsuccessful attempts to reproduce [25] chart and achieve agreement between EMPro simulation and analytical model, the T-matching analytical model was not used in our design.

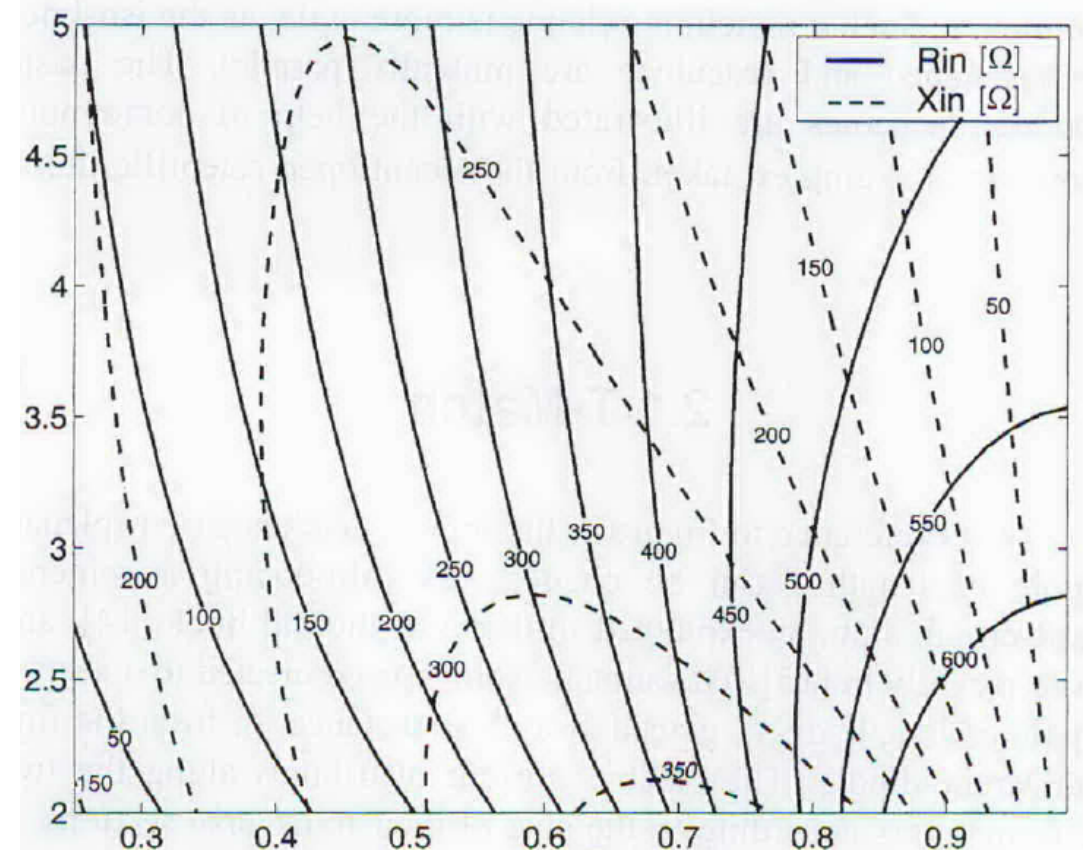

(a) 


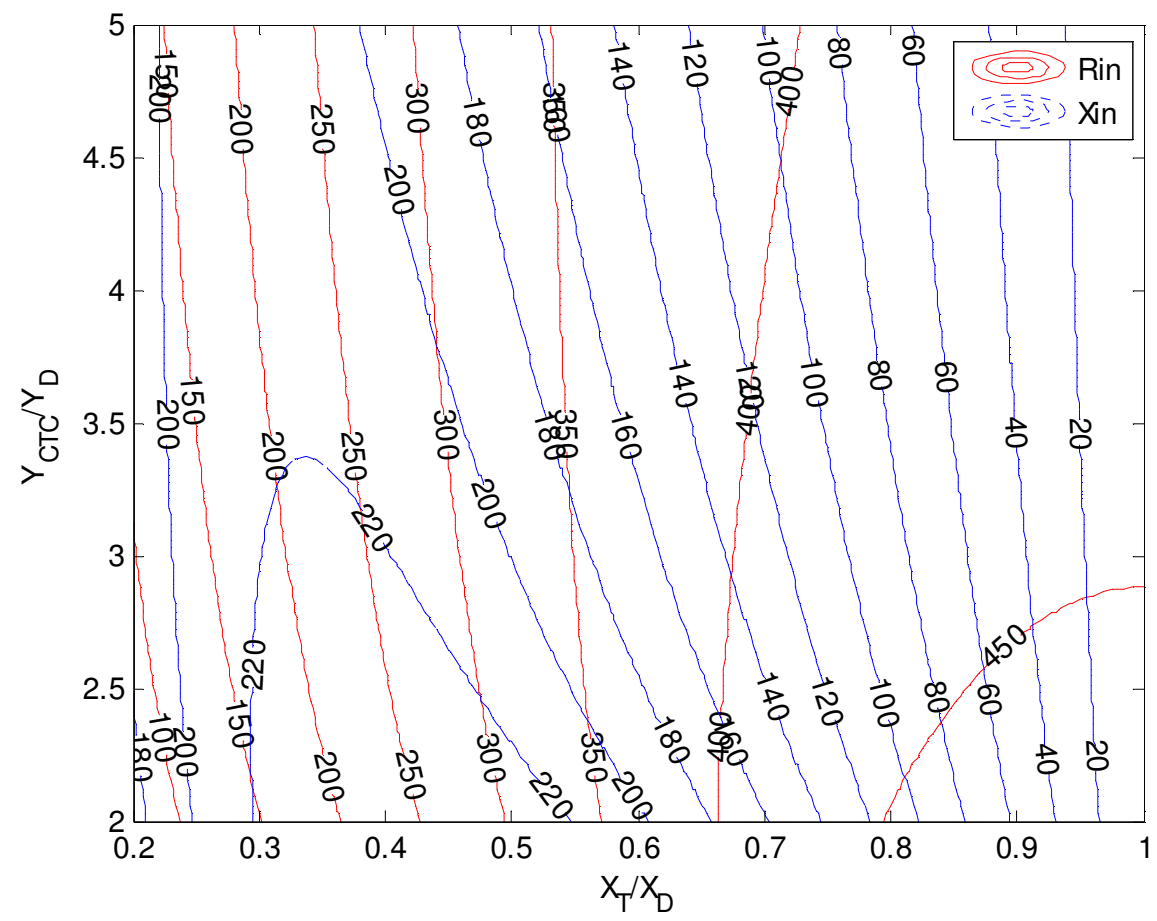

(b)

Figure 3.14: T-Matching Dimension Matching Chart (a) From Ref [25] (b) Reproduced

In addition to the Uda Model limitations for T-matching antennas, researchers also developed a passive UHF RFID tag circuit model, Figure 3.15(b), in which the Tmatching structure, Figure 3.15(a), is modeled as series and shunt inductors [15]. The circuit model was developed to facilitate impedance matching. Due to the circuit model's analytical complexity, this impedance matching method was not fully explored in this project.

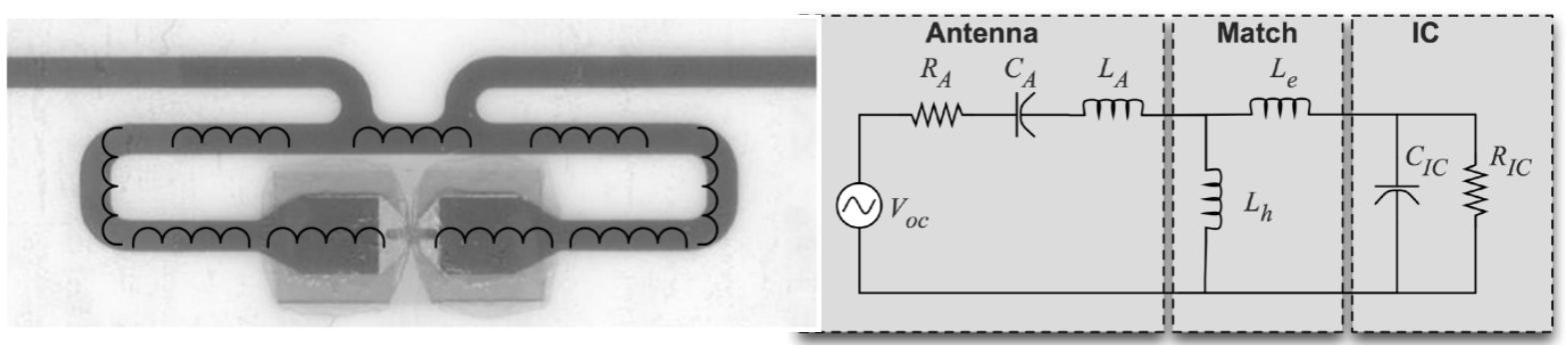

(a)

(b)

Figure 3.15: (a) T-Matching, Inductor-Based View (b) Proposed Passive UHF Tag Circuit Model [15] 


\subsubsection{Design Model Development}

The analytical folded dipole model (see Fig. 3.16) for $d<<\lambda$ is defined by [20]

$$
Z_{A N T}=\frac{4 Z_{T} Z_{D}}{Z_{T}+2 Z_{D}}
$$

where,

$$
\begin{gathered}
Z_{T}=j Z_{0} \tan \left(k \frac{L}{2}\right) \\
Z_{0}=120 \ln \left(\frac{d}{a}\right)
\end{gathered}
$$

$\mathrm{k}$ : wave number, $2 \pi / \lambda$

L: folded dipole length

d: spacing between the two dipoles

a: wire radius

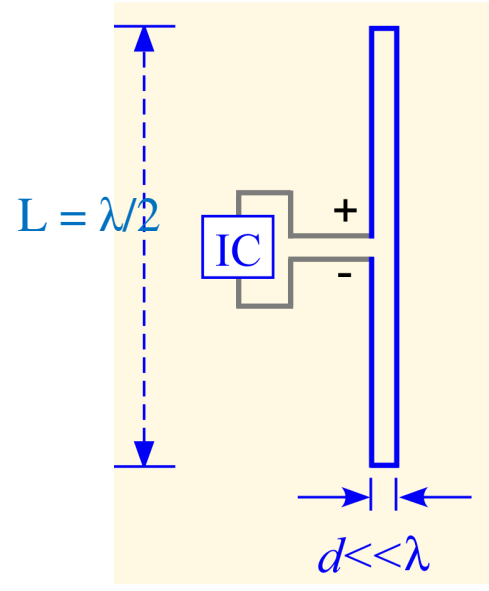

Figure 3.16: Folded Dipole Model [1]

According to equations $3.12-3.14$, a resonant folded dipole $(L=0.5 \lambda)$ provides a four-fold increase in impedance compared to a $\lambda / 2$ dipole $\left(Z_{T}=\infty\right.$ and $\left.Z_{\mathrm{ANT}}=4 Z_{\mathrm{D}}\right)$, 
which is used to reduce dipole length while matching the IC impedance. This analytical model was verified using EM simulations (see section 3.1.5.1).

To minimize the tag's conductive area, a folded dipole with a closed loop structure $[31,32]$ is adopted for the project design. The model is illustrated in Figure 3.17 along with structural dimensions. The initial design is $80 \mathrm{~mm} \times 20 \mathrm{~mm}$ to match IC impedance 22.5 - j127.8 $\Omega$. Section 3.1.5.2 describes the EM simulation optimization, including final dimensions and input impedance.

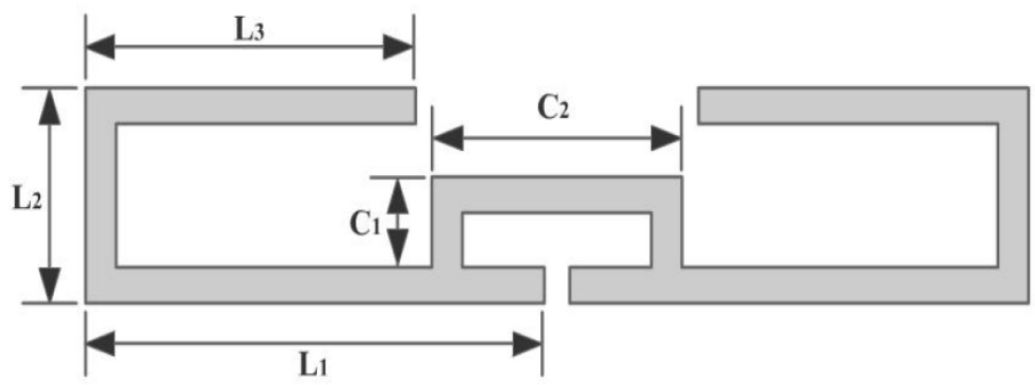

Figure 3.17: Final RFID Design Model [31]

The tag simulation design model includes five parameters:

$\mathrm{L}_{1}$ : main dipole structure length

$\mathrm{L}_{2}$ : folded dipole height

$\mathrm{L}_{3}$ : folded dipole length

$\mathrm{C}_{1}$ : closed loop structure height

$\mathrm{C}_{2}$ : closed loop structure length

\subsubsection{Tag Performance Simulation and Optimization}

To reduce optimization complexity, L1 and L2 are fixed [23] (Figure 3.7, Section 3.1.3). The folded dipole footprint is $92.2 \mathrm{~mm} \times 8.0 \mathrm{~mm}\left(2 \mathrm{~L}_{1} \times \mathrm{L}_{2}\right)$, while the dipole's trace width is matched to the Sirit IC strap width. 


\subsubsection{EMPro Model Verification}

RFID antenna design was accomplished using the EMPro 3D electromagnetic (EM) simulation tool [37]. Antenna impedance and S-parameters were calculated in EMPro using either the Finite Element Method (FEM) or Finite Difference Time Domain (FDTD) method.

To verify EMPro model accuracy, a dipole model was simulated and compared to the analytical model. The input impedance of an infinitely thin half-wave wire dipole is $73.0+j 42.5 \Omega$ [20]. If the length is slightly reduced (for example, from $0.500 \lambda$ to 0.475 $\lambda$ ), resonance (reactance $X=0 \Omega$ ) can be achieved with input impedance $70+\mathrm{j} 0 \Omega$. As the wire dipole's radius is increased, resonant length is decreased. A planar dipole (metallic strip) is simulated to model the RFID tag antennas structure. Modeling a halfwave dipole $(\lambda=327.9 \mathrm{~mm}$ at $915 \mathrm{MHz})$, the dipole impedance $(\mathrm{L}=0.5 \lambda)$ at the feed point is shown in Figure 3.18. The model defines a $0.01 \lambda$ at $915 \mathrm{MHz}$ trace width and resonance occurs at approximately $839.2 \mathrm{MHz} ; \mathrm{Z}_{\mathrm{in}}=71.39+\mathrm{j} 0 \Omega$. 


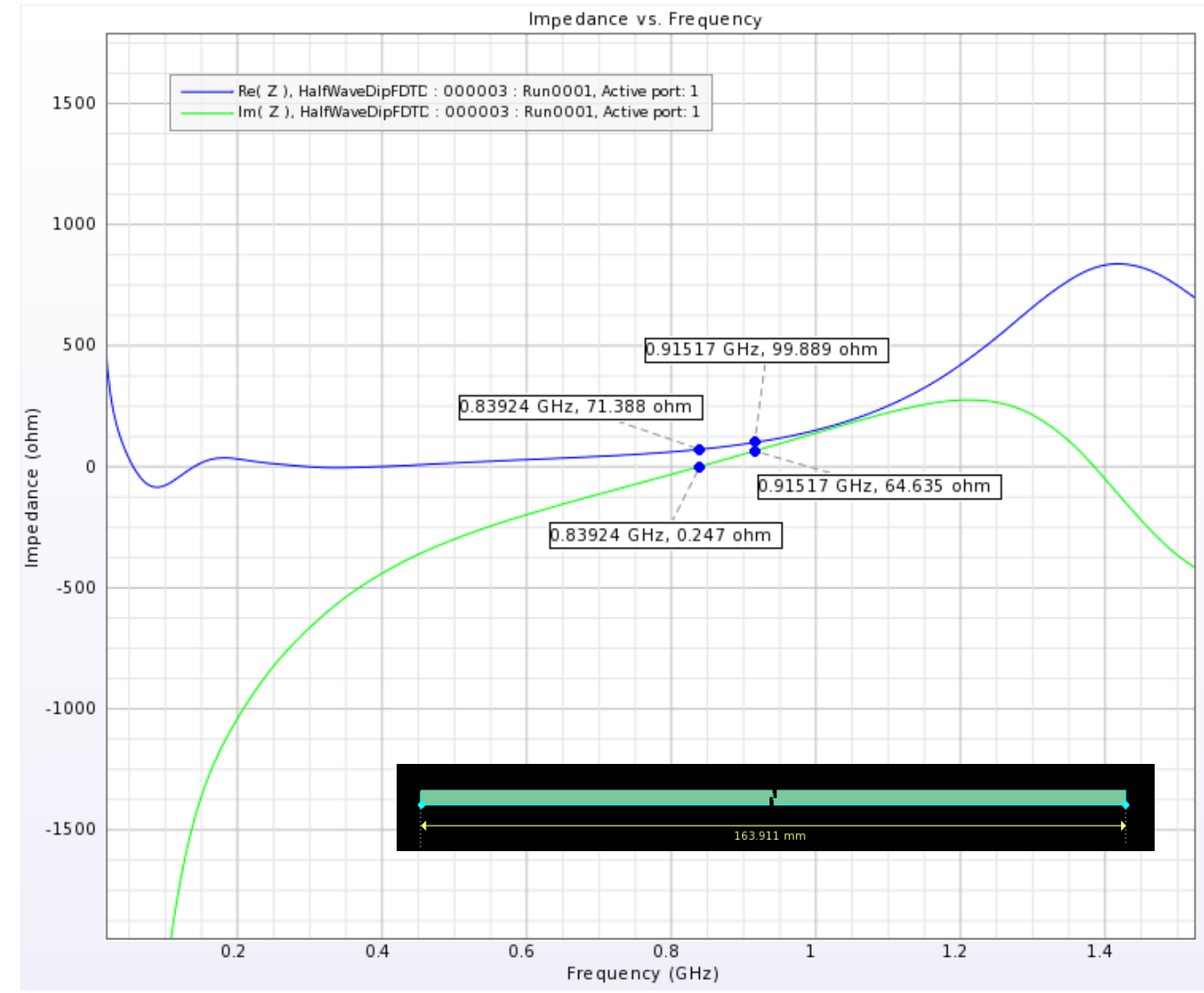

Figure 3.18: Dipole Impedance vs. Frequency $(L=0.5 \lambda)$

Decreasing dipole length to $0.455 \lambda$ at $915 \mathrm{MHz}$, resonance is achieved at 915 $\mathrm{MHz}$ as shown in Figure 3.19. The resulting real impedance is $70.4 \Omega$. 


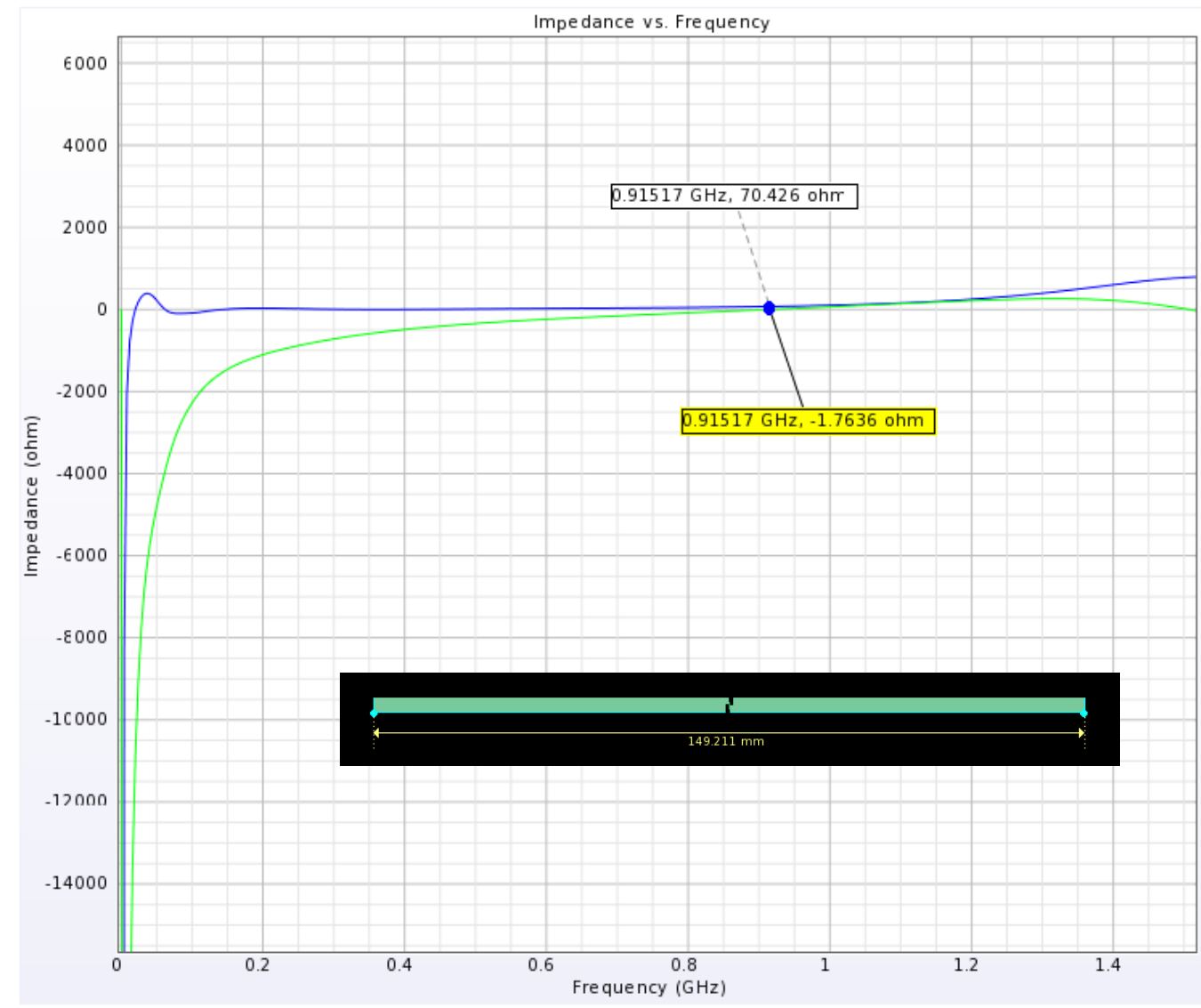

Figure 3.19: Dipole Impedance vs. Frequency $(L=0.455 \lambda)$

The folded dipole model in Section 3.1.4.3 is also used to verify simulation model accuracy. A folded dipole with $3 \mathrm{~mm}$ spacing $(\mathrm{d}=0.009 \lambda), 0.455 \lambda$ length and $0.01 \lambda$ trace width was simulated, see Figure $3.20(\mathrm{Zin}=287.44+\mathrm{j} 3.528 \Omega$ at $915 \mathrm{MHz})$. The folded dipole resistance is approximately four times its dipole version as predicted by the analytical model. 


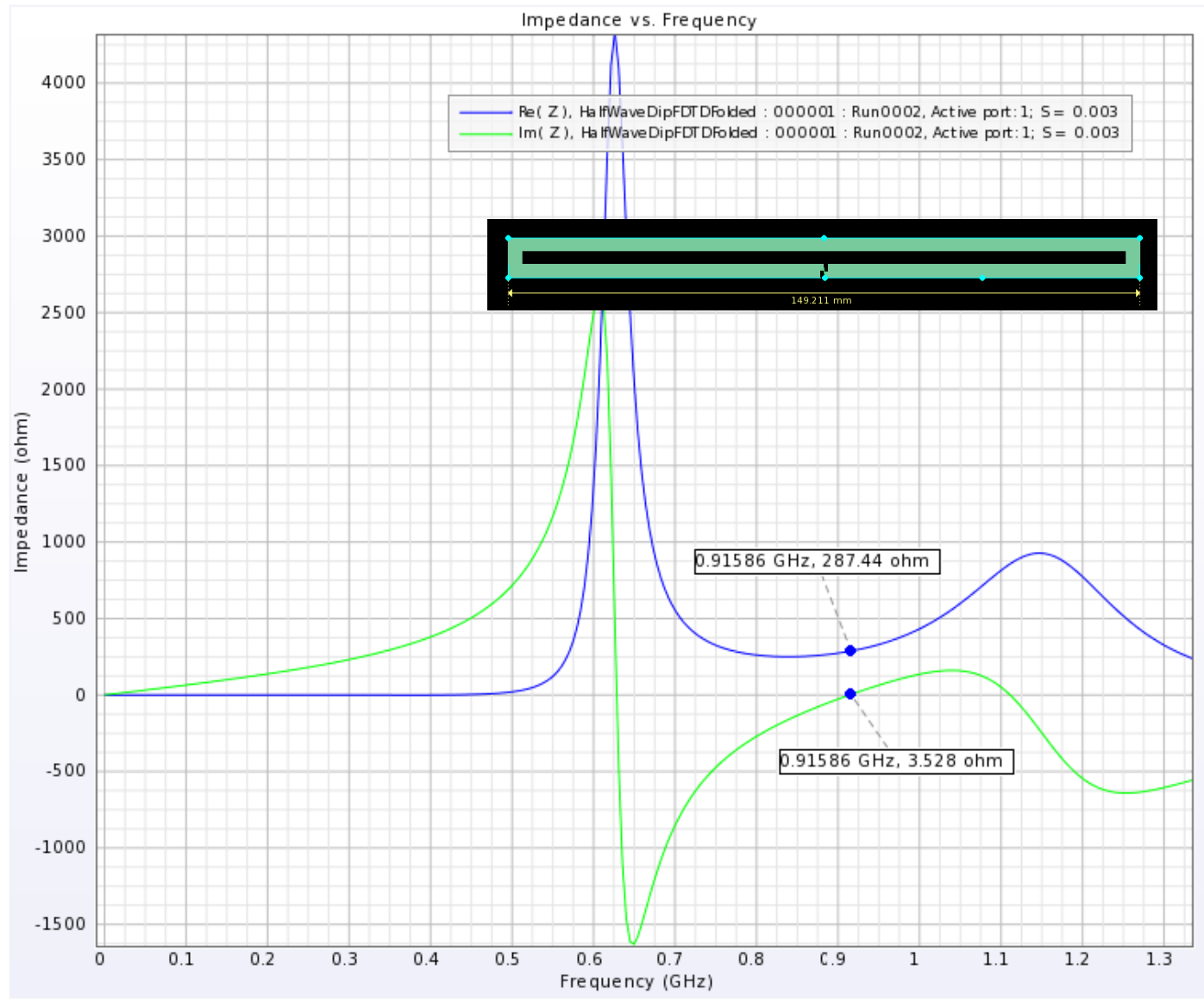

Figure 3.20: Folded Dipole Impedance vs. Frequency

\subsubsection{Tag Model Parametric Study}

To reduce simulation runtime and complexity, simulations are performed without the substrate. Omitting the substrate does not significantly affect model accuracy due to relatively low dielectric constant (3.0 to 3.9) and 50 micron thickness (see Table 3-4). To determine folded dipole length (L3) effects on antenna impedance, the outer dimension ( $92 \mathrm{~mm} \times 8 \mathrm{~mm}$ ) is maintained (Sirit tag outline) while the folded length is varied from 10 $\mathrm{mm}$ to $40 \mathrm{~mm}$. The resulting impedances are plotted in Figure 3.22 and Figure 3.23. These results indicate that the folded dipole length substantially affects reactance $(-600 \Omega$

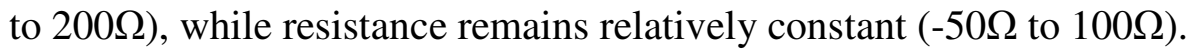




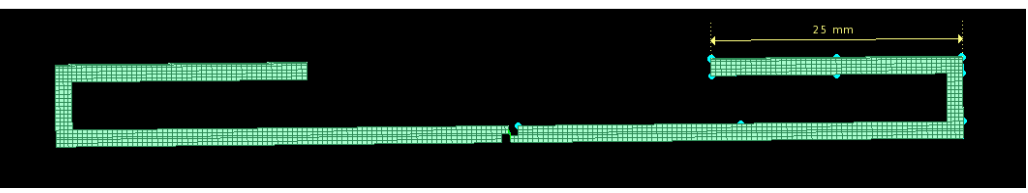

Figure 3.21: Folded Dipole, 25 mm Folded Length

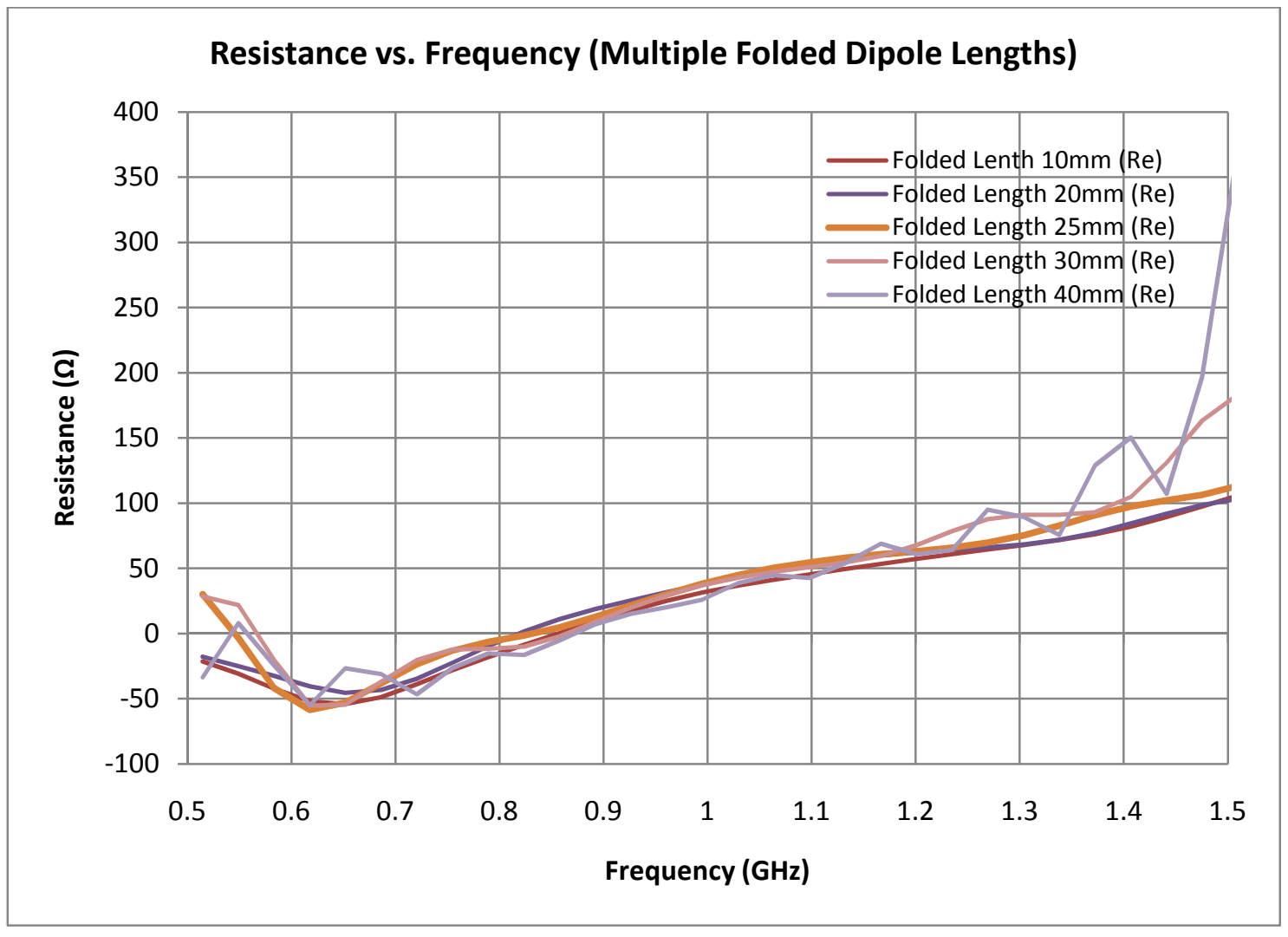

Figure 3.22: Resistance vs. Frequency, Multiple Folded Dipole Lengths 


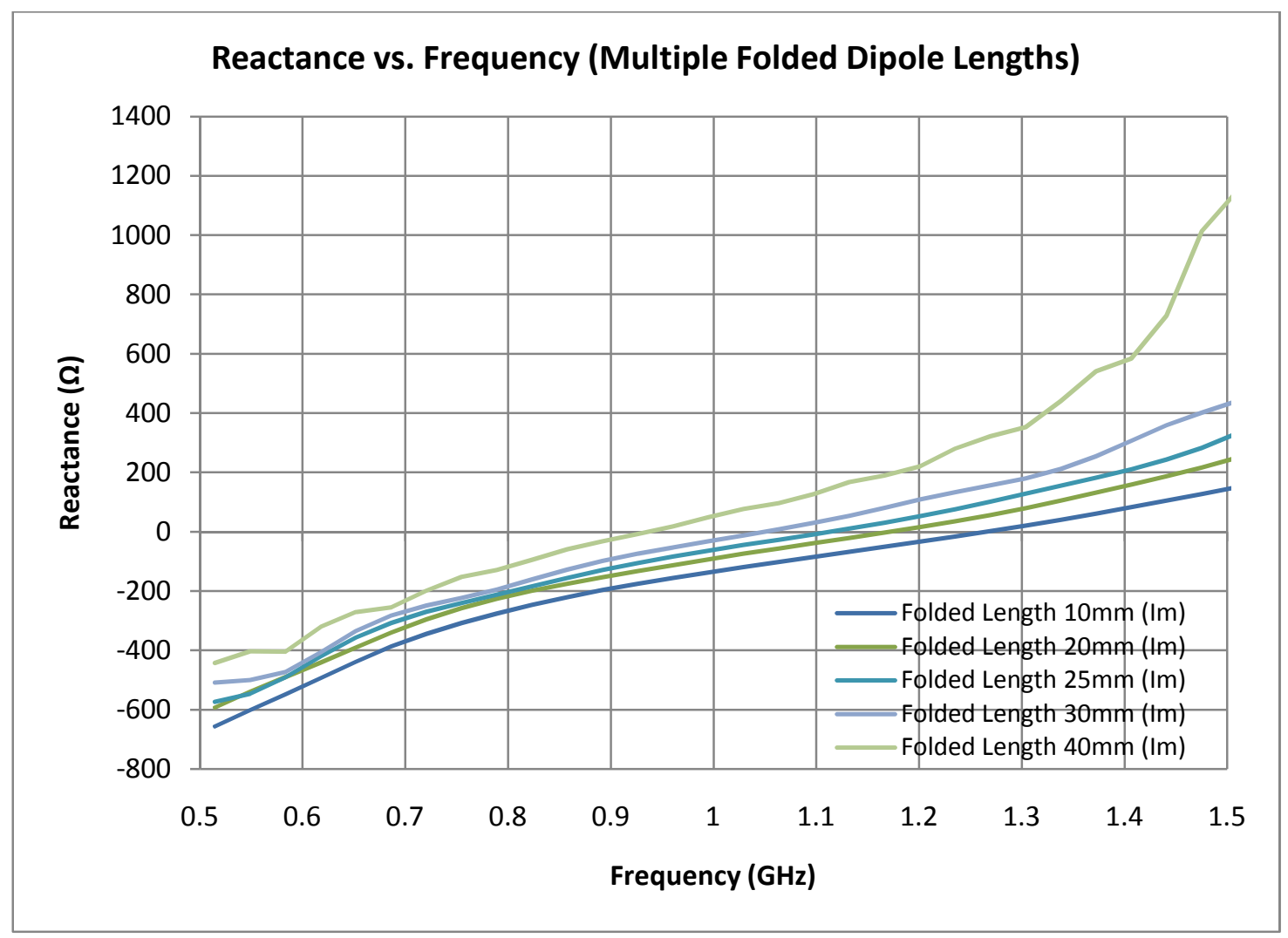

Figure 3.23: Reactance vs. Frequency, Multiple Folded Dipole Lengths

The closed loop structure model shown in Figure 3.24 indicates increasing resistance and reactance values for loop widths of $3 \mathrm{~mm}$ to $15 \mathrm{~mm}$. Increasing the loop height from $0.5 \mathrm{~mm}$ to $3.5 \mathrm{~mm}$ also increases both resistance and reactance. Parametric trends were used to facilitate impedance matching.

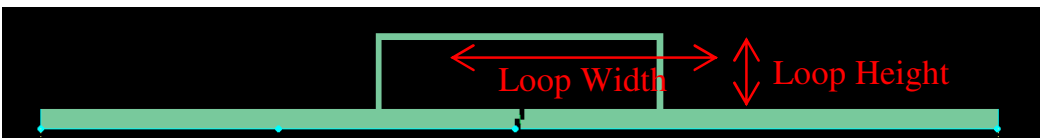

Figure 3.24: EMPro Model, Closed Loop Structure 
The closed loop structure trace width was also varied to conjugate match the antenna impedance to the Monza 3 IC input impedance.

\subsubsection{Reference Tag (Sirit RSI-674) Simulation}

The Sirit RSI-674 was simulated (FDTD) to determine desired impedance values. Figure 3.25 shows the Sirit RFID tag (without substrate) input impedance frequency response. The impedance at $930 \mathrm{MHz}$ is $6.88+\mathrm{j} 140.92 \Omega$.

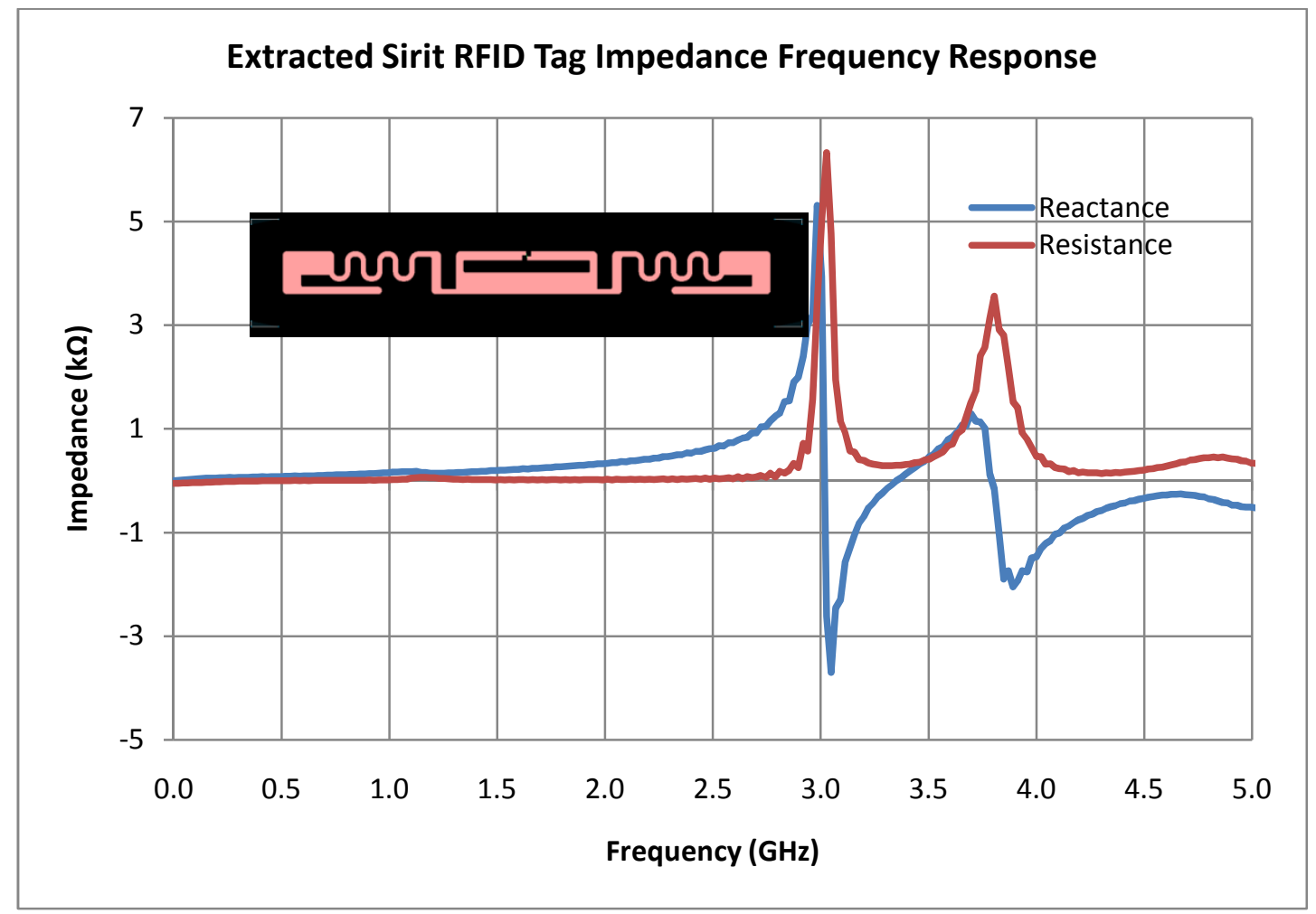

Figure 3.25: Extracted Sirit RFID Tag Impedance Frequency Response

Table 3-3 summarizes Monza 3 IC input impedances values from multiple sources. Although there should only be one impedance value, it is difficult to determine the most accurate value. As a consequence, three designs were created based on the three 
specified or simulated antenna impedances. The simulated impedance at $930 \mathrm{MHz}$ was the closest available value to $915 \mathrm{MHz}$ in the EMPro FDTD simulation.

Table 3-3: Monza 3 IC Recommended Antenna Input Impedance

\begin{tabular}{|l|c|c|}
\hline Sources & Frequency & Recommended Antenna Impedance \\
\hline Monza 3 Datasheet [12] & $915 \mathrm{MHz}$ & $32+\mathrm{j} 216 \Omega$ \\
\hline Measured Value [22] & $912 \mathrm{MHz}$ & $15+\mathrm{j} 180 \Omega$ \\
\hline $\begin{array}{l}\text { EMPro Simulated Values (Sirit } \\
\text { Commercial Tag) }\end{array}$ & $930 \mathrm{MHz}$ & $7+\mathrm{j} 141 \Omega$ \\
\hline
\end{tabular}

\subsubsection{Final RFID Tag Design}

The PET substrate was inserted to verify performance and apply design refinements as necessary. The input impedance frequency responses for the three tag designs are shown in Figure 3.26 and final design impedances at 915MHz are summarized in Table 3-4. Key structural dimensions for Designs 1 through 3, shown in Figure 3.27, are summarized in Table 3-5. Several other dimensions, defined in Figure 3.27(a), were also optimized.

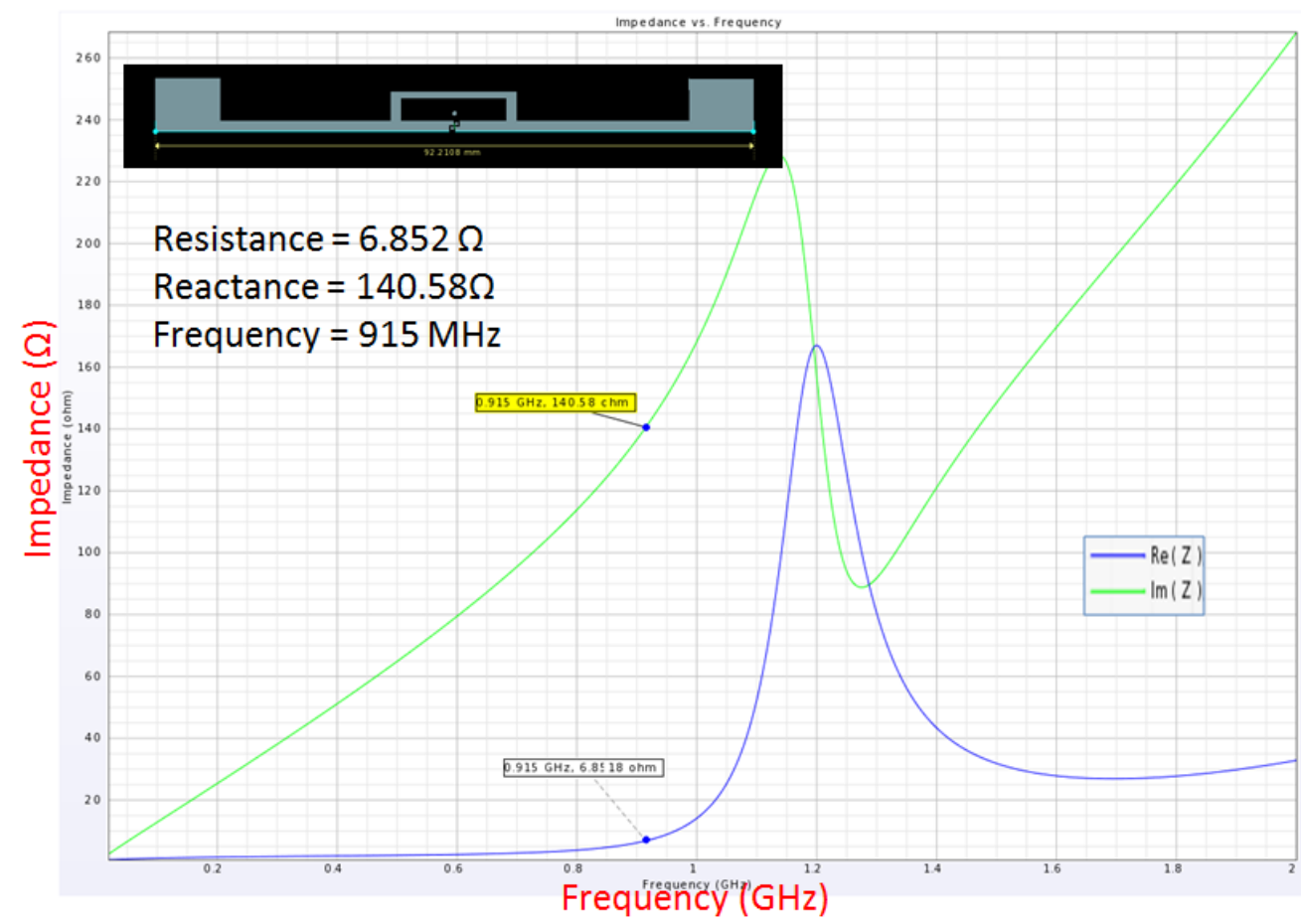

(a) 


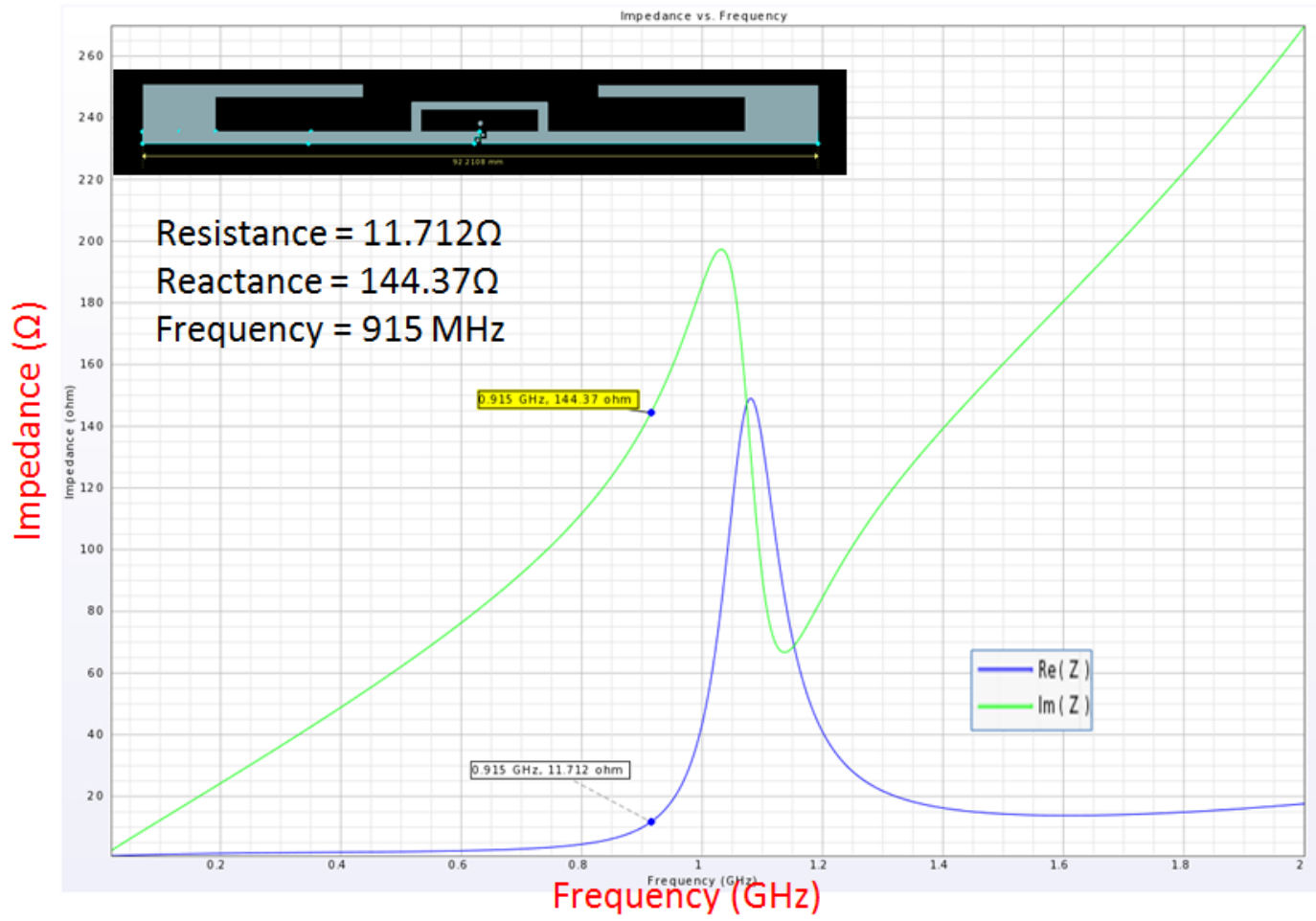

(b)

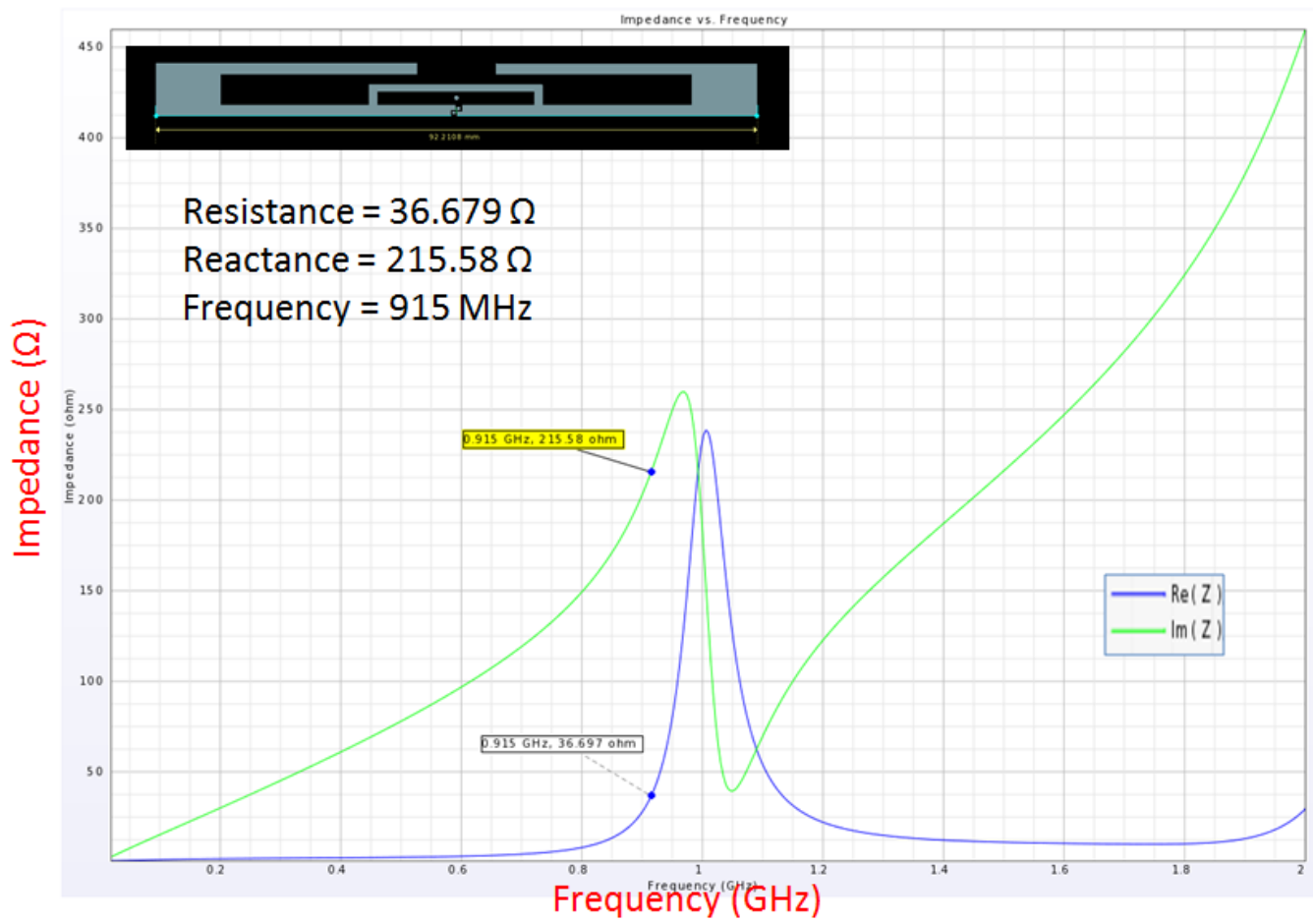

(c)

Figure 3.26: Tag Design Input Impedance Frequency Responses (a) Design \#1, (b) Design \#2, (c) Design \#3 
Tip-Loading Width

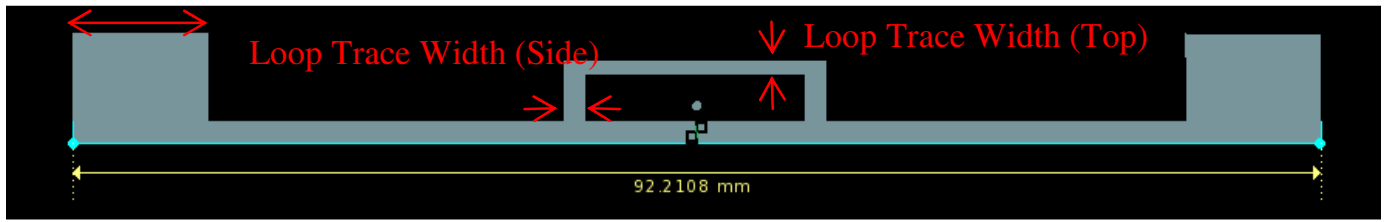

(a)

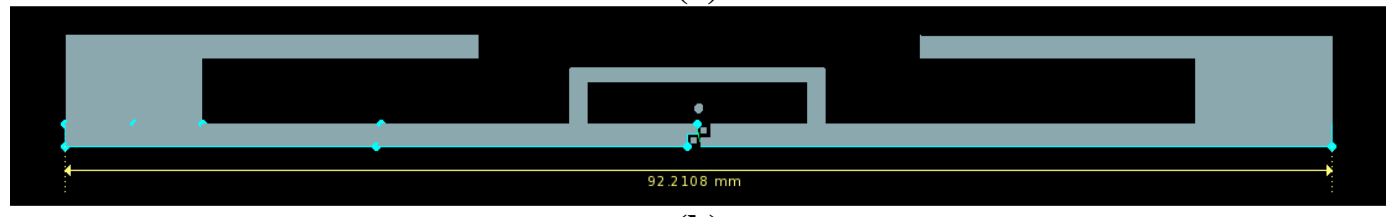

(b)

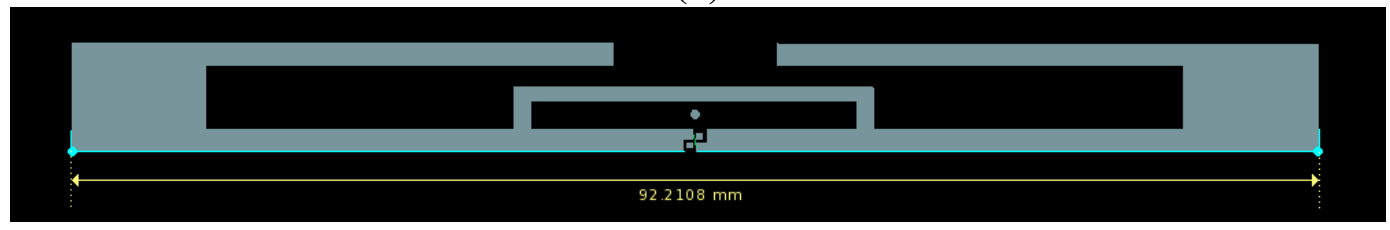

(c)

Figure 3.27: Final RFID Antenna Designs (a) Design \#1, (b) Design \#2, (c) Design \#3

Table 3-4: Final Design Impedance (at 915MHz)

\begin{tabular}{|c|c|c|}
\hline Design & $\begin{array}{c}\text { Simulated Impedance } \\
\text { (without substrate) }\end{array}$ & $\begin{array}{c}\text { Simulated Impedance (with } \\
\text { PET substrate) }\end{array}$ \\
\hline 1 & $6.025+\mathrm{j} 132.68 \Omega$ & $6.852+\mathrm{j} 140.58 \Omega$ \\
\hline 2 & $10.194+\mathrm{j} 136.66 \Omega$ & $11.712+\mathrm{j} 144.37 \Omega$ \\
\hline 3 & $28.468+\mathrm{j} 200.61 \Omega$ & $36.697+\mathrm{j} 215.58 \Omega$ \\
\hline
\end{tabular}

Table 3-5: Final Tag Designs Key Structural Dimensions (mm)

\begin{tabular}{|c|c|c|c|}
\hline Design & 1 & 2 & 3 \\
\hline Closed Loop Structure Height $\left(\mathbf{C}_{\mathbf{1}}\right)$ & 3.40 & 3.00 & 2.10 \\
\hline Closed Loop Structure Length $\left(\mathbf{C}_{\mathbf{2}}\right)$ & 8.08 & 8.00 & 12.00 \\
\hline Folded Dipole Length $\left(\mathbf{L}_{\mathbf{3}}\right)$ & 10.00 & 30.00 & 40.00 \\
\hline Folded Dipole Height $\left(\mathbf{L}_{\mathbf{2}}\right)$ & 8.00 & 8.00 & 8.00 \\
\hline Main Dipole Structure Length $\left(\mathbf{L}_{\mathbf{1}}\right)$ & 46.10 & 46.10 & 46.10 \\
\hline Tip-Loading Width & 10.00 & 10.00 & 10.00 \\
\hline Loop Trace Width (Top) & 1.00 & 1.00 & 1.00 \\
\hline Loop Trace Width (Side) & 1.30 & 1.65 & 1.30 \\
\hline
\end{tabular}

\subsubsection{Prototype Construction and Test}

This section describes measurement techniques used to obtain the RFID tag antenna's input impedance. The tag design prototypes were constructed on FR4 with copper traces to enable antenna terminal connections to a test fixture (described in 
Section 3.1.6.1). Corresponding EMPro models (copper traces on FR4 substrate) were simulated and compared to measured values. Section 3.1.6.1 describes RFID tag impedance measurement techniques [33-40]. Selected measurement procedures are described in Section 3.1.6.2, while Section 3.1.6.3 provides comparisons between measured and simulated impedance values.

\subsubsection{RFID Antenna Impedance Measurement Method}

Four methods - single-ended probe, balun connected probe, imaging, and differential probe have been used by researchers to measure RFID tag antenna impedance [33].

The RFID strap has identical pads on both side of the IC which transfer energy between the antenna and IC. The RFID strap serves as a balanced feed as shown in Figure 3.28. The symbol $\mathrm{V}$ in the figure denotes applied or received voltage. With equal magnitude and opposite polarity voltages on the strap's two sides, the strap's center plane acts as a virtual ground.

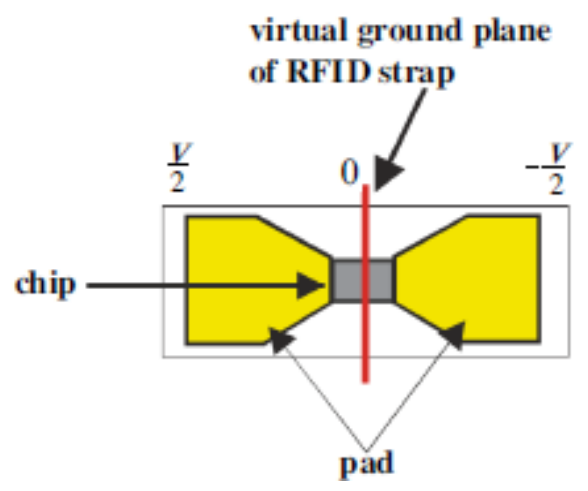

Figure 3.28: RFID Strap and Virtual Ground Plane [38] 
An SMA connector extension [39] is applied to the coaxial cable to form a singleended probe: see Figure 3.29. Similar to coaxial ports, a single-ended probe is an unbalanced test port which is not suitable for measuring a balanced structure. If an unbalanced test port is connected to a balanced antenna, source currents on the two antenna radiators are unequal due to unequal impedances at the two ports. Unequal currents flowing through the balanced structure compromise measurement accuracy. Most RFID tag antennas are balanced; hence, single-ended probes are not appropriate for direct RFID impedance measurements.
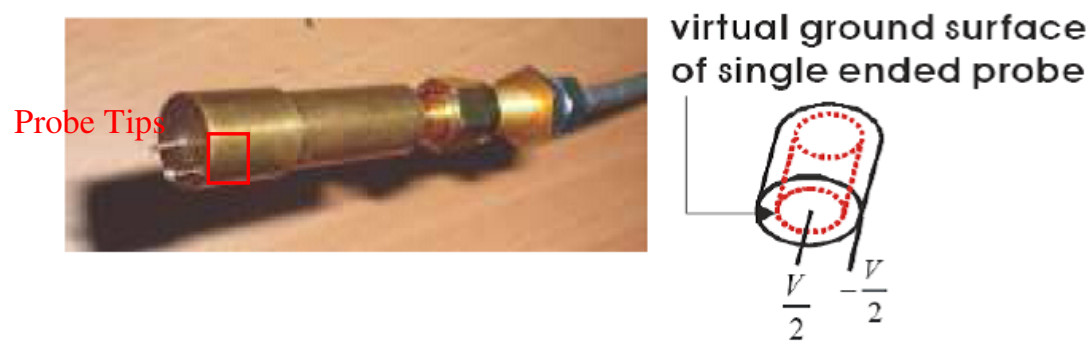

Figure 3.29: RFID Impedance Measurement, Commercially Available Single-Ended Probe (V= voltage) [39]

Balanced antennas, such as dipoles, are measured through a balun, which produces equal current flow to both antenna radiators, e.g.: two dipole arms. Figure 3.30 shows a commercial wire balun. Prediction accuracy depends on the wire balun selection and how well it transfers energy between the balanced antenna and unbalanced test port over the test frequency range. 


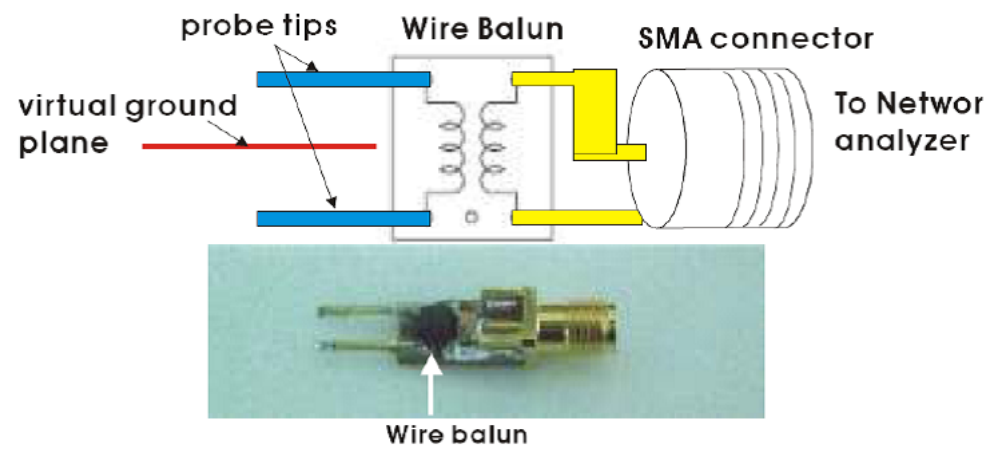

Figure 3.30: RFID Impedance Measurement, Commercial Wire Balun [39]

The imaging method is another technique to measure balanced antennas. Half of a symmetrical balanced antenna is mounted on a ground plane. According to the image theory, a mirror image is created symmetric to the ground plane and acts as an independent antenna radiator. Doubling the measured monopole impedance yields the balanced dipole impedance. Calculation accuracy depends on ground plane size; a finite ground plane creates an image within a limited range of observation angles.

The differential method has been considered by several researchers $[36,38,41]$. The classic method [34] uses an S-parameter technique involving a two-port model [33, 34].

1. Construct test fixture (Figure 3.31) to connect antenna port (RFID tag location) to VNA (Vector Network Analyzer) test ports 1 and 2: see Figure 3.32

2. Two-port calibrate VNA to test fixture input (balanced) ports.

3. Short-circuit test fixture and apply port extension.

4. Measure all four S-parameters.

5. Calculate antenna impedance, (3.16) 
The general form [33]:

$$
Z_{d}=\frac{2 Z_{0}\left(1-S_{11} S_{22}+S_{12} S_{21}-S_{12}-S_{21}\right)}{\left(1-S_{11}\right)\left(1-S_{22}\right)-S_{12} S_{21}}
$$

For symmetrical balanced antennas, $S_{11}=S_{22}, S_{12}=S_{21}$, equation (3.15) is simplified to

$$
Z_{d}=\frac{2 Z_{0}\left(1-S_{11}^{2}+S_{21}^{2}-2 S_{12}\right)}{\left(1-S_{11}\right)^{2}-S_{21}^{2}}
$$

Equation (3.16) is further simplified to [41]:

$$
Z_{d}=2 Z_{0} \frac{1+S_{11}-S_{21}}{1-S_{11}+S_{21}}
$$

The original test fixture [34] is composed of two $50 \Omega$ microstrip lines on back-toback printed circuit boards (PCB) as shown in Figure 3.31(a). The fixture is calibrated to the microstrip line terminations at the top. A later technique uses a coaxial cable fixture similar to Figure 3.31(b) and standard VNA calibration kits [37].

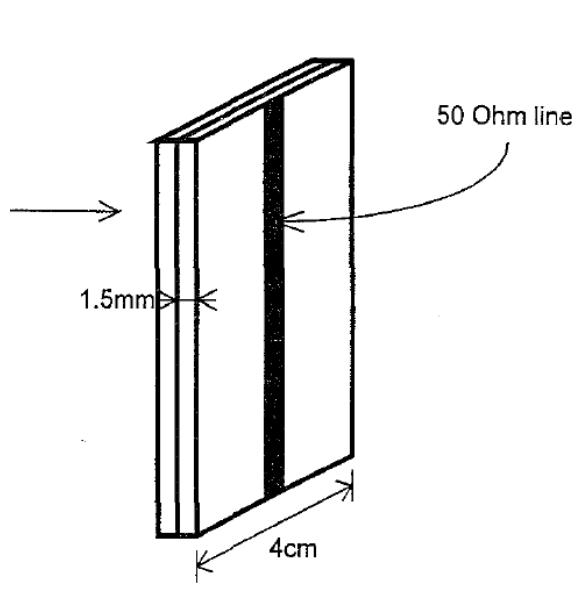

(a)

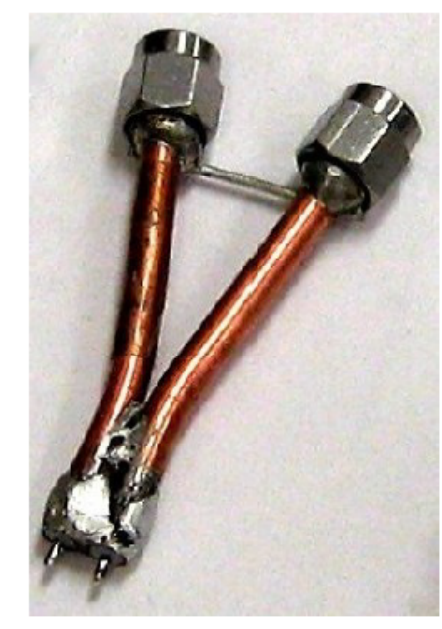

(b)

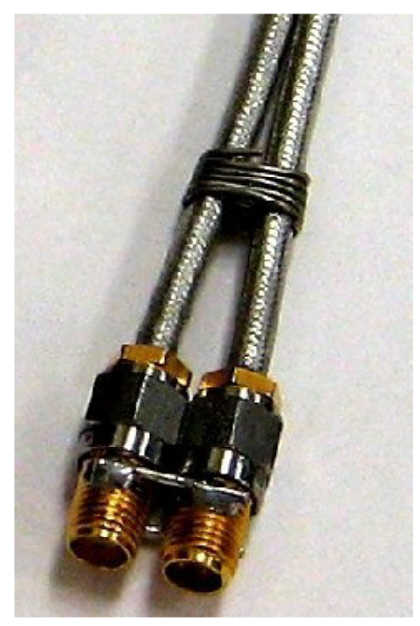

(c)

Figure 3.31: Differential Method Test Fixture (a) Classic Fixture [34] (b) Modern Fixture \#1 [40] (c) Modern Fixture \#2 [40]

Figure 3.32 illustrates the measurement setup to obtain required S-parameters.

This technique establishes the VNA calibration plane as shown using calibration 
standards [37]. The coax cable is characterized for attenuation $\alpha$ and phase delay $\beta$ values, which are used to de-embed the test fixture and minimize the S-parameter measurement errors. Detailed procedures are described in [37].

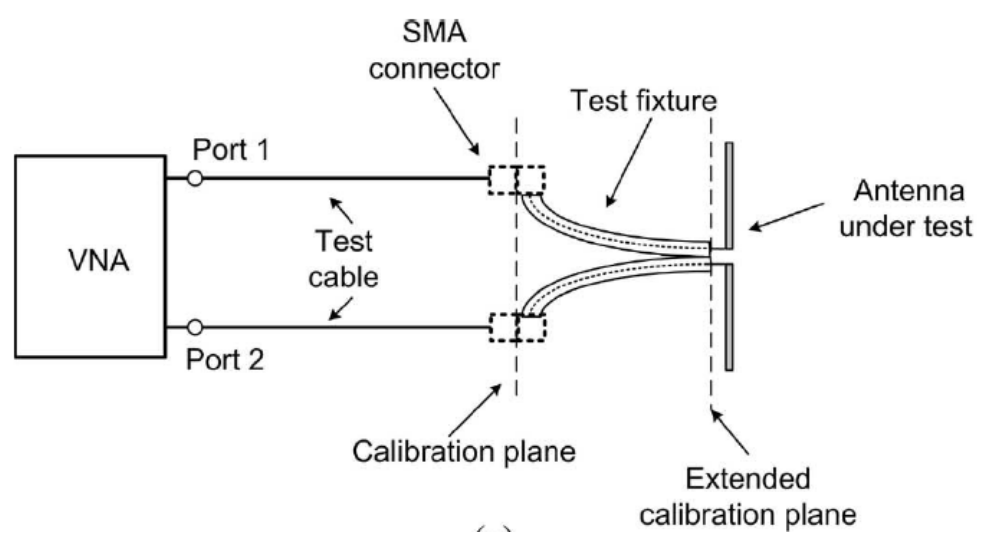

Figure 3.32: RFID S-Parameter Measurement Setup

In [38], a full two-port "SOLT" (short-open-load-through) calibration at the probe tips (extended calibration plane) is proposed; however, this method requires a nonstandard calibration kit [38]. The test fixture in Figure 3.31(c) is a modification to Figure 3.31(b) which allows calibration standard application at the probe tips directly [40]; however, this modified test fixture requires SMA connections at the antenna ports.

Finally, several authors [33, 35-36] utilize the VNA port extension option to shift the reference plane to the probe tips. Port extension is a phase shift compensation feature provided by VNA vendors to extend the measurement reference plane. Electrical delay is applied to all S-parameters associated with each port. To apply port extensions, the fixture must be open or short circuited at the probe tips. The short-circuit configuration is recommended since open circuit conditions are difficult to achieve due to radiation. A short-circuit can be formed by soldering the two probe tips to the ground connection (outer conductor) [33]. 


\subsubsection{RFID Tag Antenna Impedance Measurements}

The fabricated RFID tag with silver conductive traces on PET substrate cannot be attached directly to the text fixture because the substrate cannot withstand the soldering process. Instead, the three antennas and Sirit designs were fabricated on 31 mil thick FR4 substrate with copper traces patterns produced by an LPKF S62 Prototyper. The fabricated tags and test fixture are shown in Figure 3.33. The test fixture contains two 10cm length semi-rigid coaxial cables (UT-085C-TP-M17, Micro-Coax) and two SMA straight plugs.

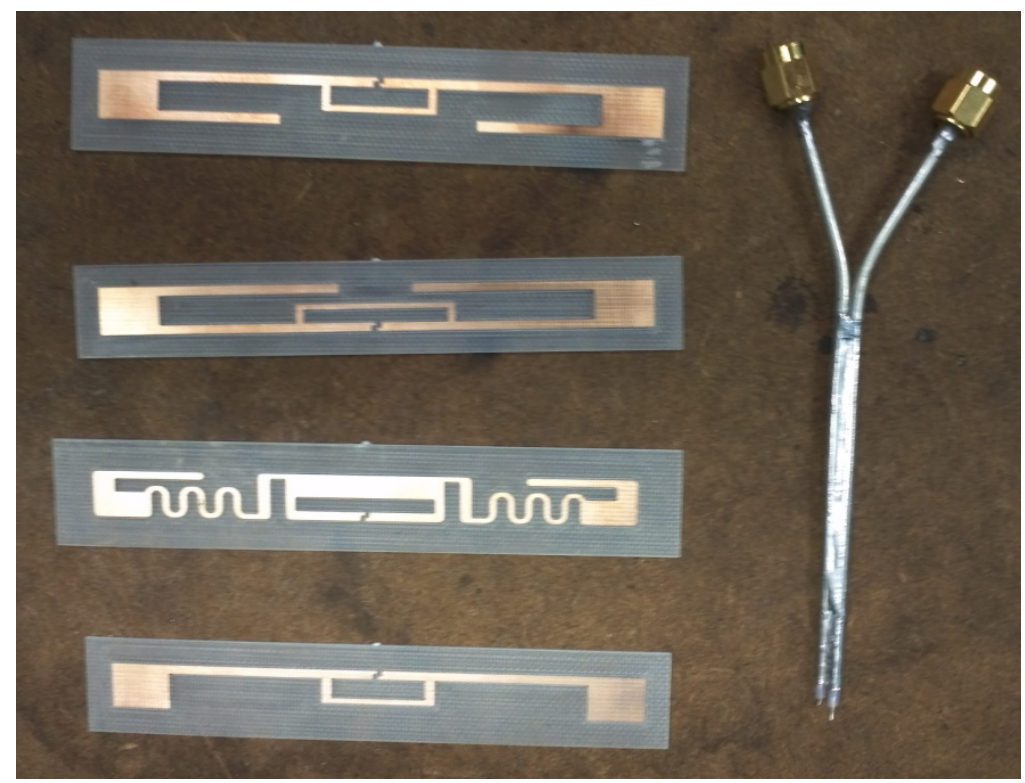

Figure 3.33: RFID Tags Fabricated on FR4 board and Test Fixture

Measurements were performed inside the anechoic chamber to prevent external RF signal interference and surrounding environment reflections. The VNA (HP8720C Network Analyzer) was calibrated to the SMA test port plane over the frequency range 
$850 \mathrm{MHz}$ to $950 \mathrm{MHz}$. The short-circuited test fixture (Figure 3.34) was then connected to the VNA's cable.

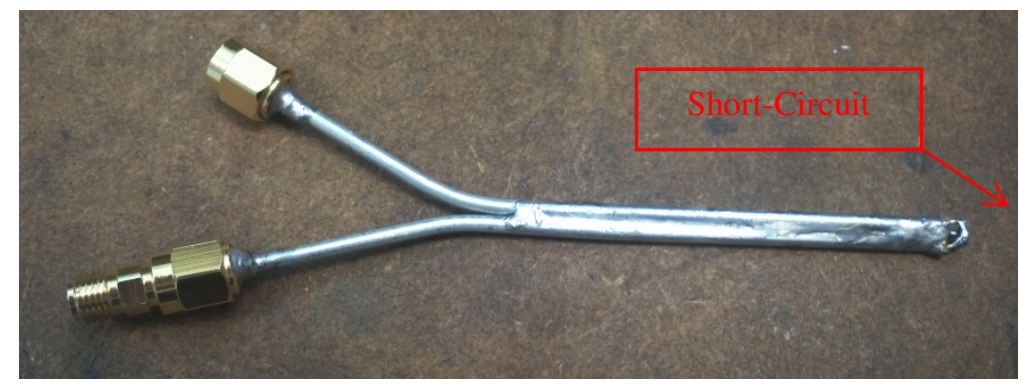

Figure 3.34: Test Fixture, Short Circuit Configuration

Port extensions are then applied to both Ports 1 and 2. Figure 3.35 shows $\mathrm{S}_{11}$ Smith Chart measurements before and after port extensions. The short-circuit measurement indicates a maximum of $900 \mathrm{~m} \Omega$ and $200 \mathrm{~m} \Omega$ for resistance and reactance. A port extension length of $180.72 \mathrm{~mm}$ was applied to Port $1,175.73 \mathrm{~mm}$ to Port 2. This approximates twice the test fixture's physical length; accounts for incident and reflected wave test fixture traversal.

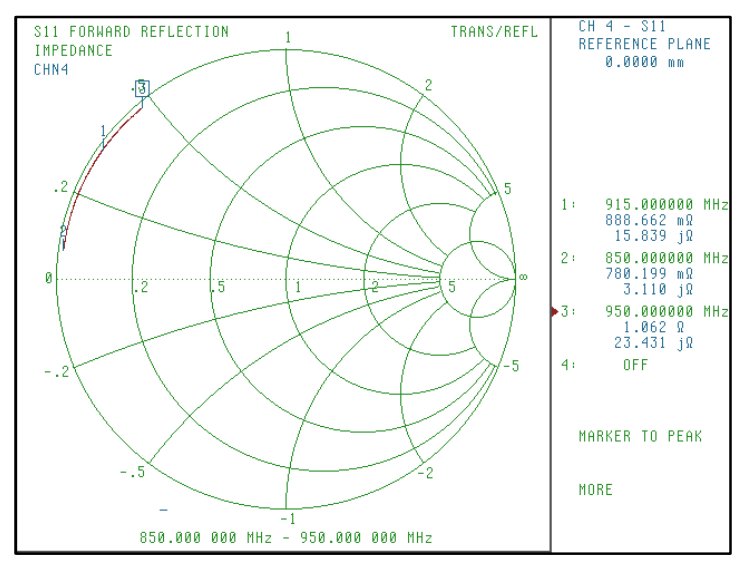

(a)

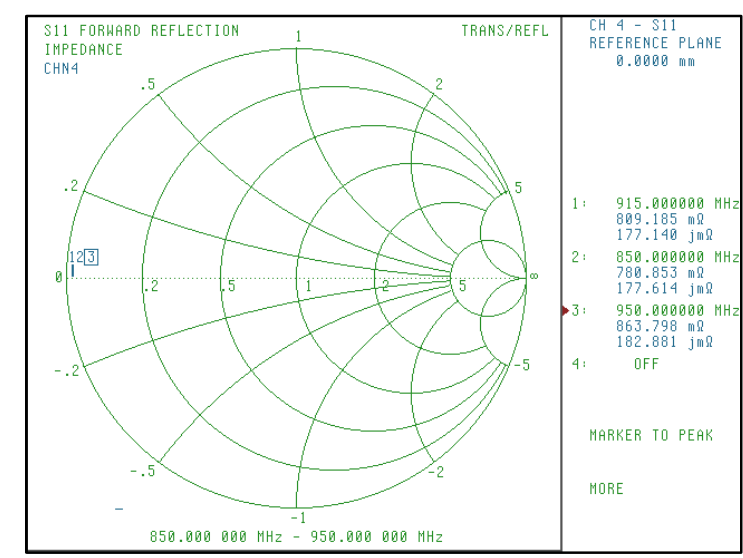

(b)

Figure 3.35: $S_{11}$ (a) Without Port Extension and (b) With Port Extension 
Finally, the antenna under test was mounted onto the text fixture with solder connections, Figure 3.36 (a) and the test fixture's SMA ports were connected to the VNA cables.

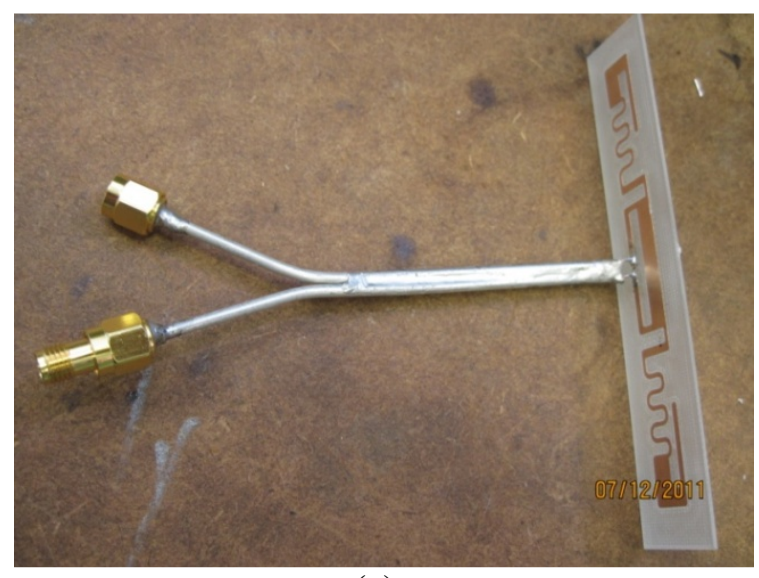

(a)

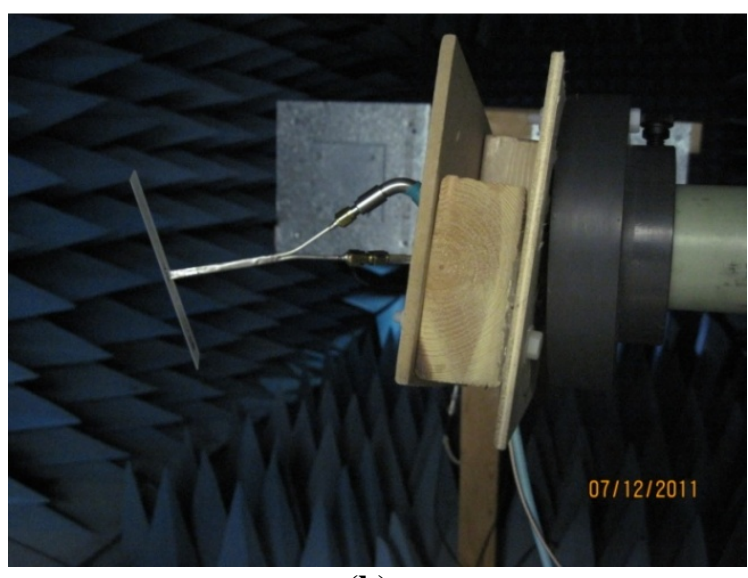

(b)

Figure 3.36: (a) Tag Antenna Under Test Mounted on Test Fixture (b) Measurement Setup Inside Anechoic Chamber

\subsubsection{Measured vs. Simulated Results}

Measured and simulated results were plotted for the three designed and Sirit tags in Figures 3.37 through 3.40. 


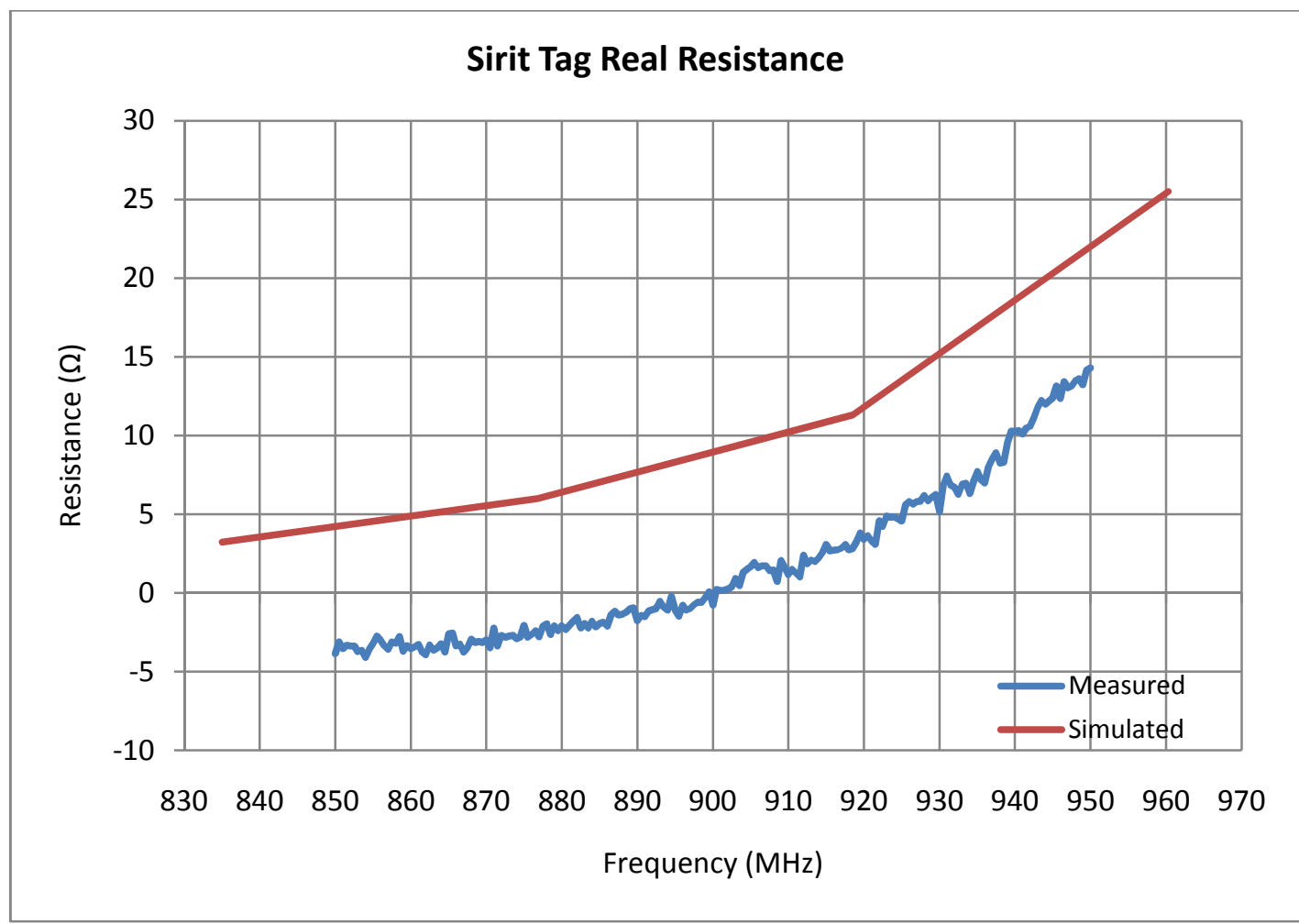

(a)

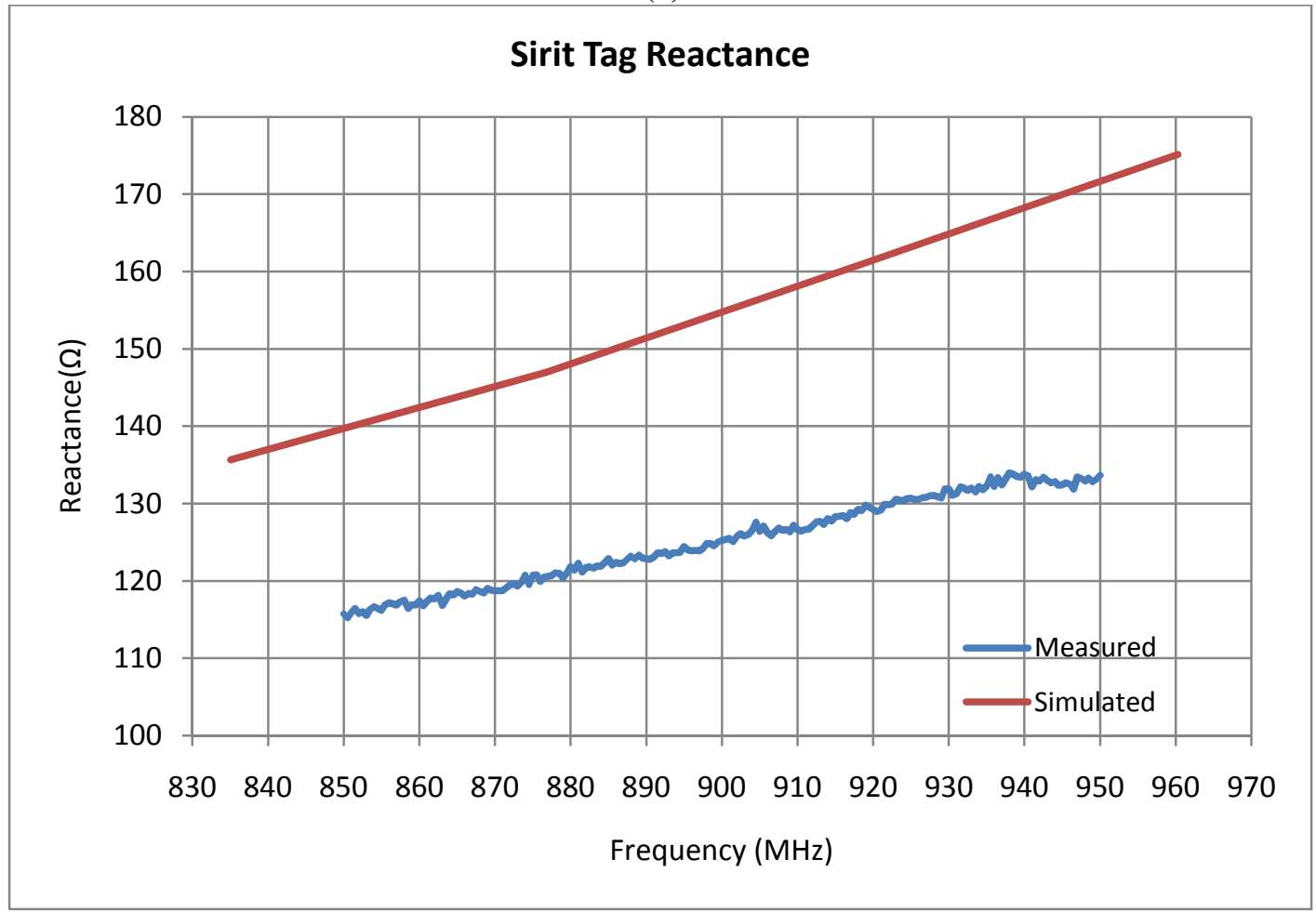

(b)

Figure 3.37: Sirit Tag Impedance, Measured vs. Simulated (a) Resistance (b) Reactance 


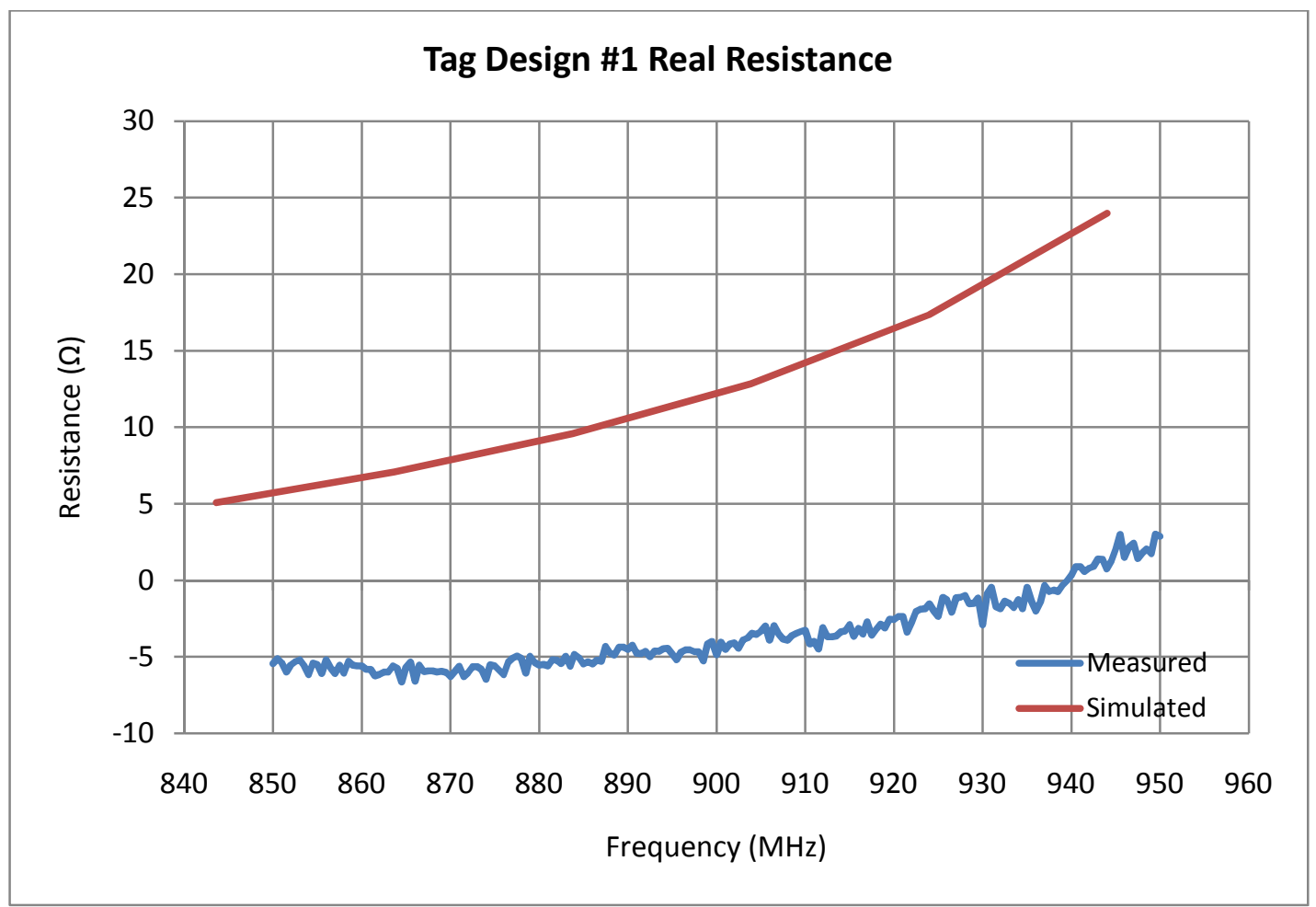

(a)

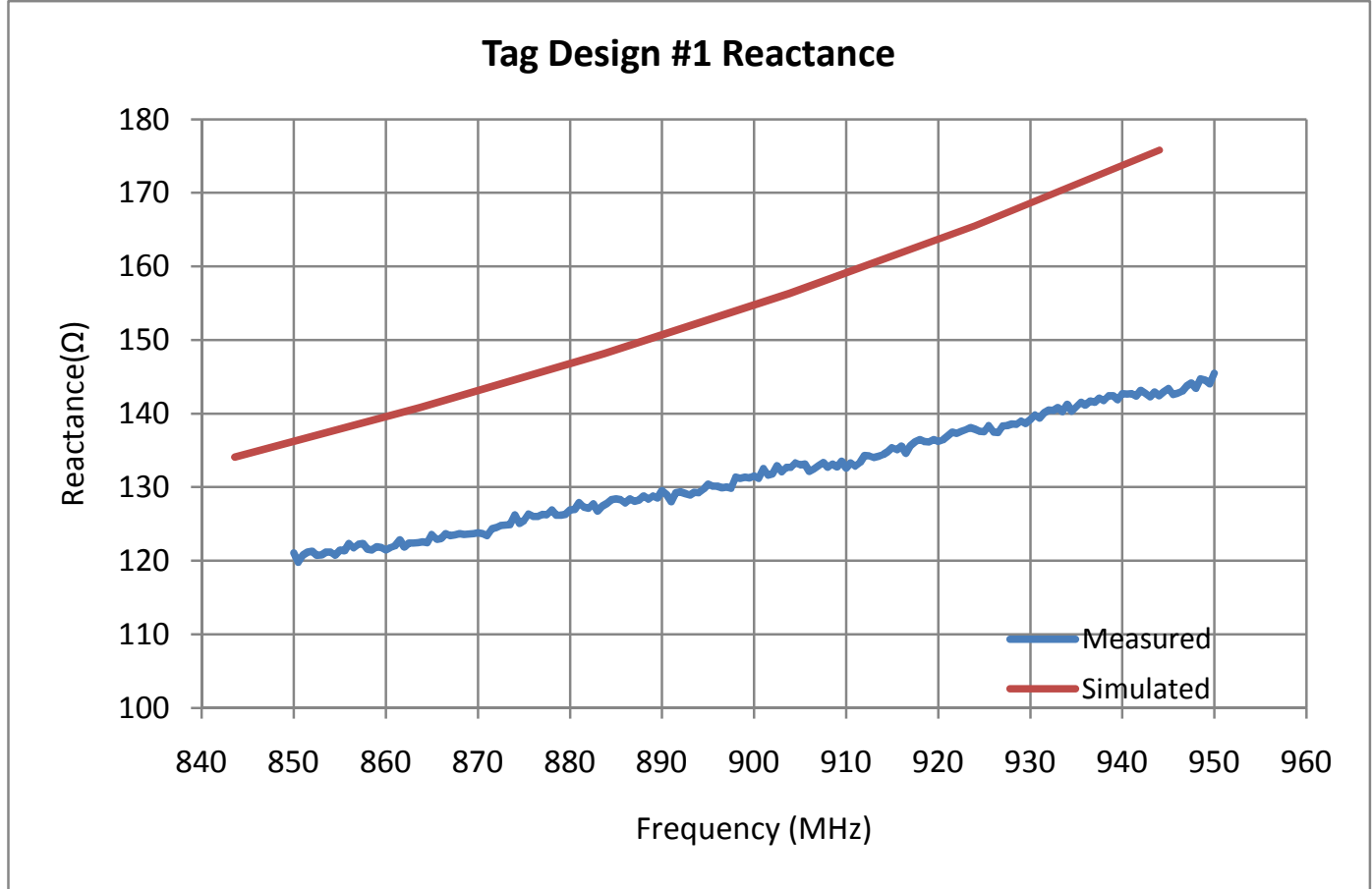

(b)

Figure 3.38: Tag Design \#1 Impedance, Measured vs. Simulated (a) Resistance (b) Reactance 


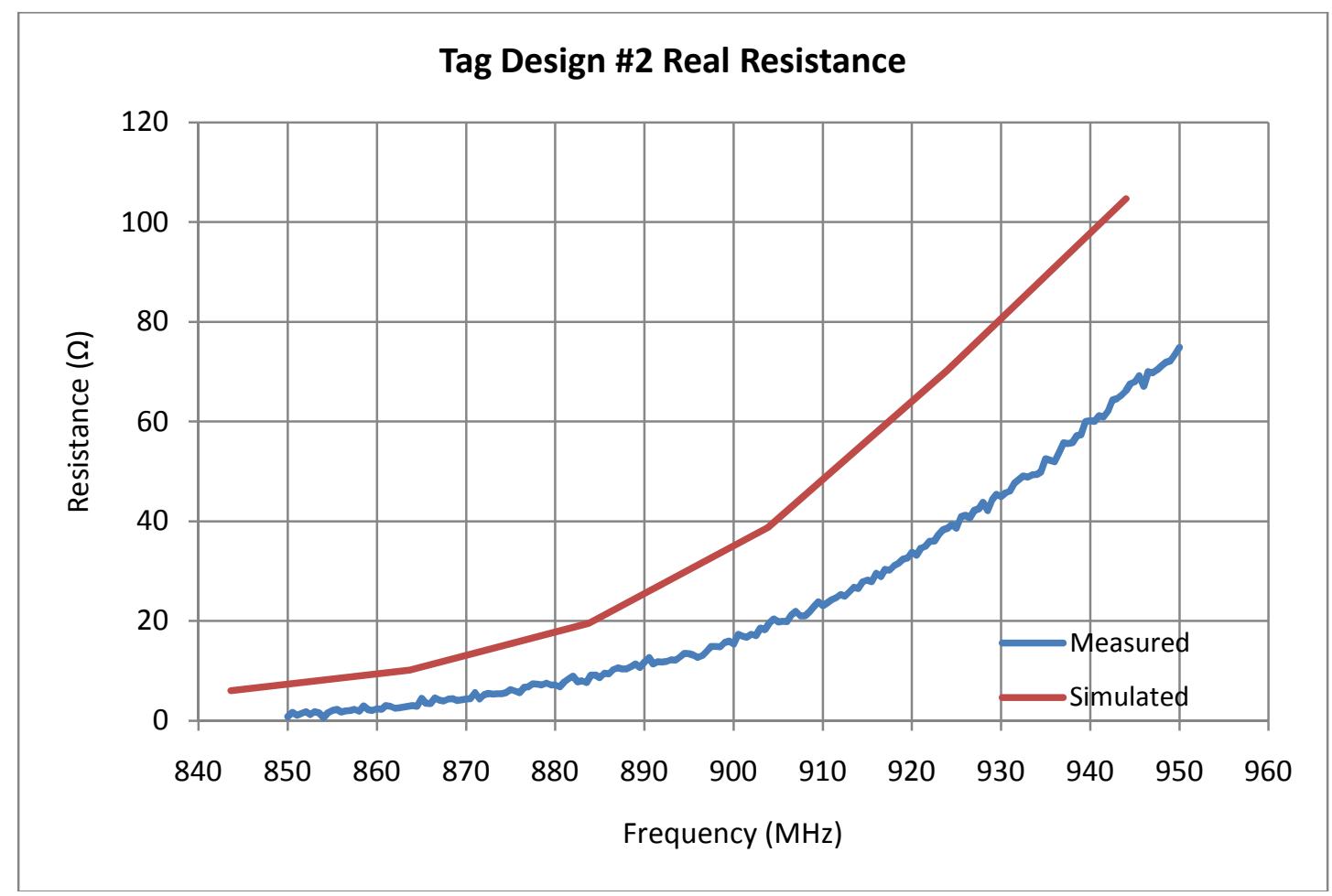

(a)

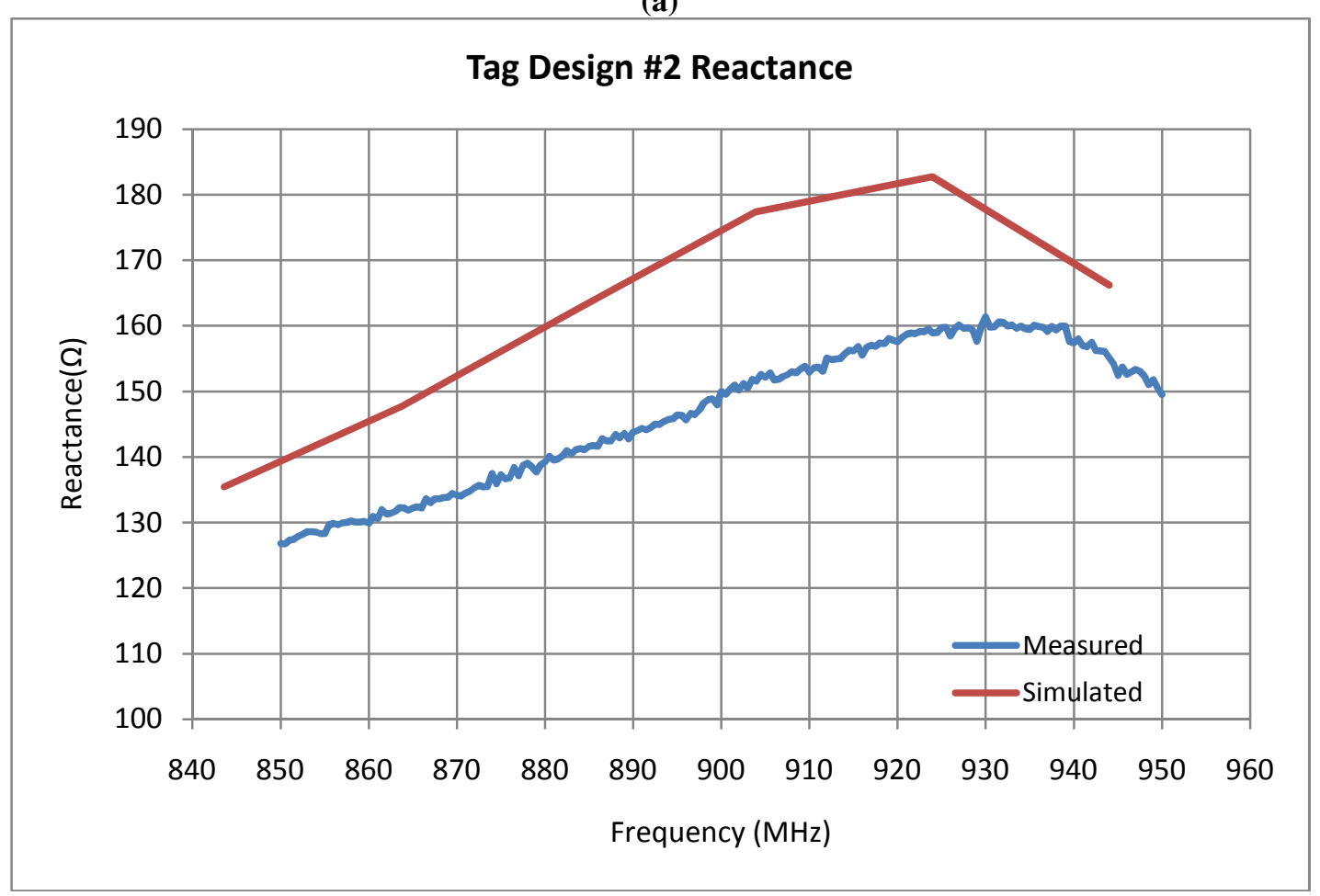

(b)

Figure 3.39: Tag Design \#2 Impedance, Measured vs. Simulated (a) Resistance (b) Reactance 


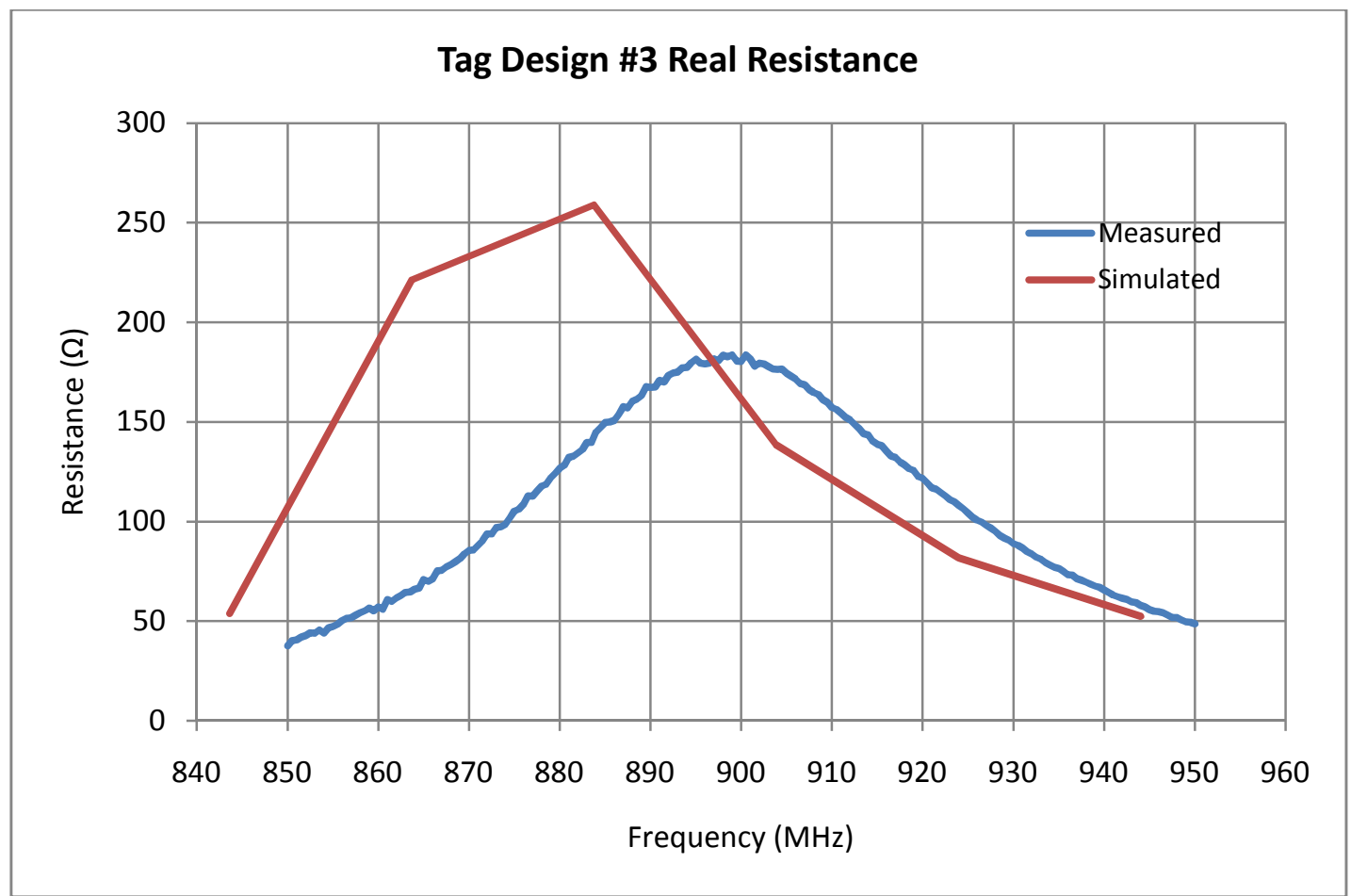

(a)

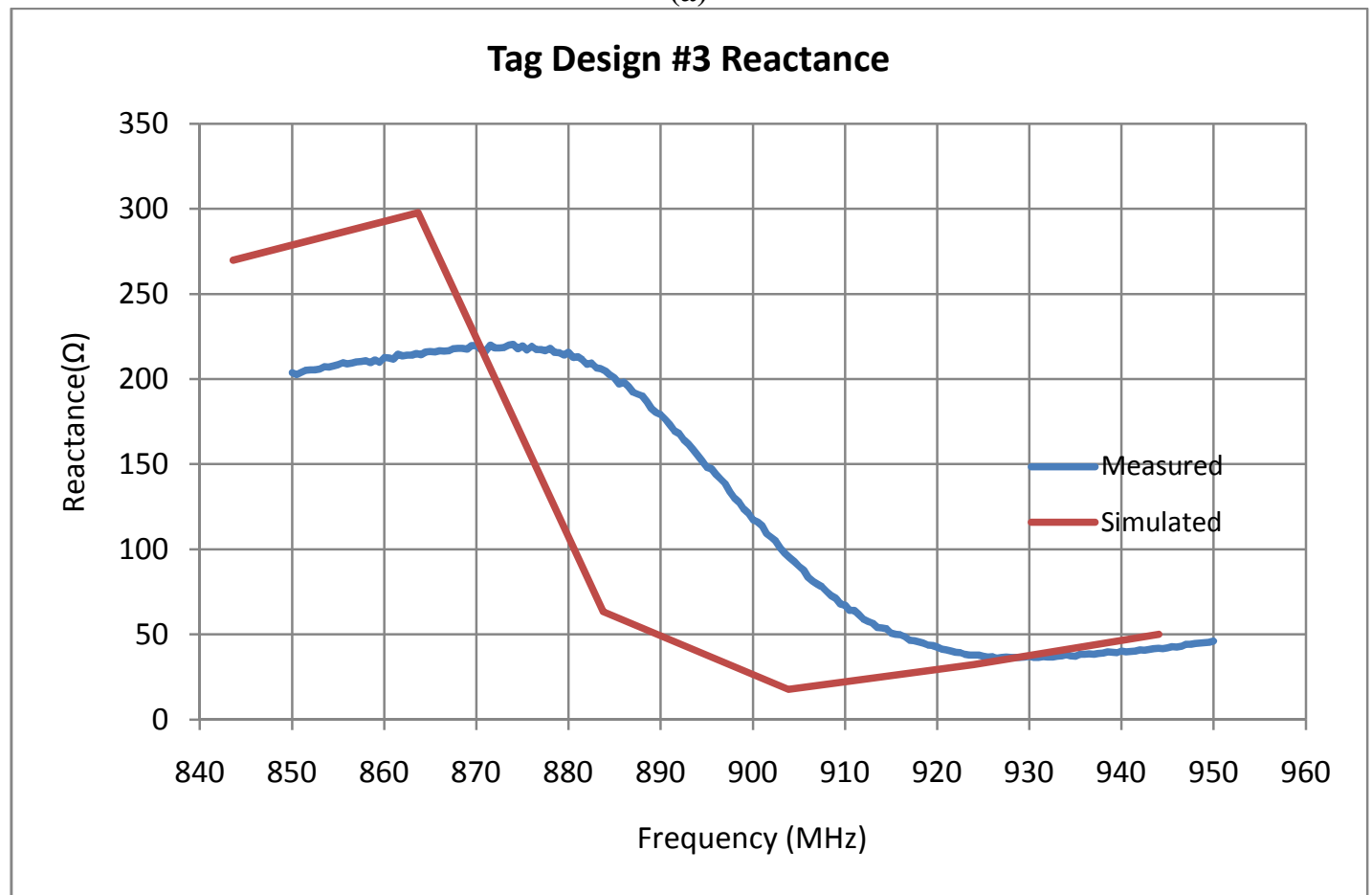

(b)

Figure 3.40: Tag Design \#3 Impedance, Measured vs. Simulated (a) Resistance (b) Reactance 
In general, both the real and imaginary component simulated and measured frequency responses have similar trends. The measured data has a relatively constant offset (100 $\Omega$ to $300 \Omega)$ from simulations. The actual cause for the offset cannot be determined. External sources could not be the cause because signal levels of $0 \mathrm{dBm}$ are required to create the observed impedance offsets. 


\section{UHF RFID TAG OPERATION VERIFICATION}

The RFID tags were fabricated according to the procedures in Chapter 2. This Chapter illustrates the tags' performance by measuring read range as defined in Section

\subsection{1.}

Section 3.1.1 defines the read range equation; however, it does not describe the measurement method. Section 4.1 describes the methods used to measure read range inside the anechoic chamber and the measurement setup. Section 4.2 discusses read range results used to validate fabrication processes (effect of conductive vs. clear tape and lamination). Section 4.3 presents data measured in the anechoic chamber for the designed tags while Section 4.4 shows patch antenna gain (TX antenna) using EMPro simulations. Finally, data from Section 4.3 along with gain extracted in Section 4.4 are used to calculate RFID tag read range reported in Section 4.5.

\subsection{Read Range Measurement Description}

Tag range measurements are conducted in the anechoic chamber to minimize the $\mathrm{RF}$ interference. The measurement setup requires a fixed distance between the reader antenna and tag, and controllable reader transmit power, which is decreased until the tag cannot be read. This value represents the minimum level required for tag communications. Read range $\left(\mathrm{r}_{\mathrm{tag}}\right)$ can be derived using equation (3.1) from reader-tag distance $(\mathrm{d})$, minimum transmit power $\left(\mathrm{P}_{\min }\right)$ and transmit antenna gain $\left(\mathrm{G}_{\text {reader }}\right)$. 
The minimum threshold power delivered to the tag antenna is

$$
P_{\text {tagmin }}=P_{\min } \frac{G_{\text {reader }} G_{\text {tag }} \lambda^{2}}{(4 \pi d)^{2}}
$$

Read range is typically defined by the IC's read sensitivity, the maximum tag read distance with the reader operating at $\mathrm{EIRP}=\mathrm{P}_{\text {reader }} \mathrm{G}_{\text {reader }}$ (effective isotropic radiated power). The power delivered to the tag antenna is

$$
P_{\text {tagreadsensitivity }}=P_{\text {reader }} G_{\text {reader }}\left(\frac{\lambda}{4 \pi r}\right)^{2}=\operatorname{EIRP}\left(\frac{\lambda}{4 \pi r}\right)^{2}
$$

Power received by the tag is the minimum power required to operate the IC. Therefore,

$$
P_{\text {tagmin }}=P_{\text {tagreadsensitivity }}
$$

Equating relations (4.1) and (4.2) and solving for read range (r) yields

$$
r_{\text {tag }}=d \sqrt{\frac{E I R P}{P_{\text {min }} G_{\text {reader }}}}
$$

The original anechoic chamber setup proposed by [42] is shown in Figure 4.1 while Figure 4.2 illustrates the modified configuration (tag center to TX/RX pair center alignment). This modification eliminates requirements for a counter-weight to maintain TX/RX antenna pair balance. To utillze the RFID reader's (Alien ALR-9800) full attenuator range (up to $12.8 \mathrm{~dB}$ ), the distance between the RFID tag and TX/RX antennas is reduced from 45in to 39in. 


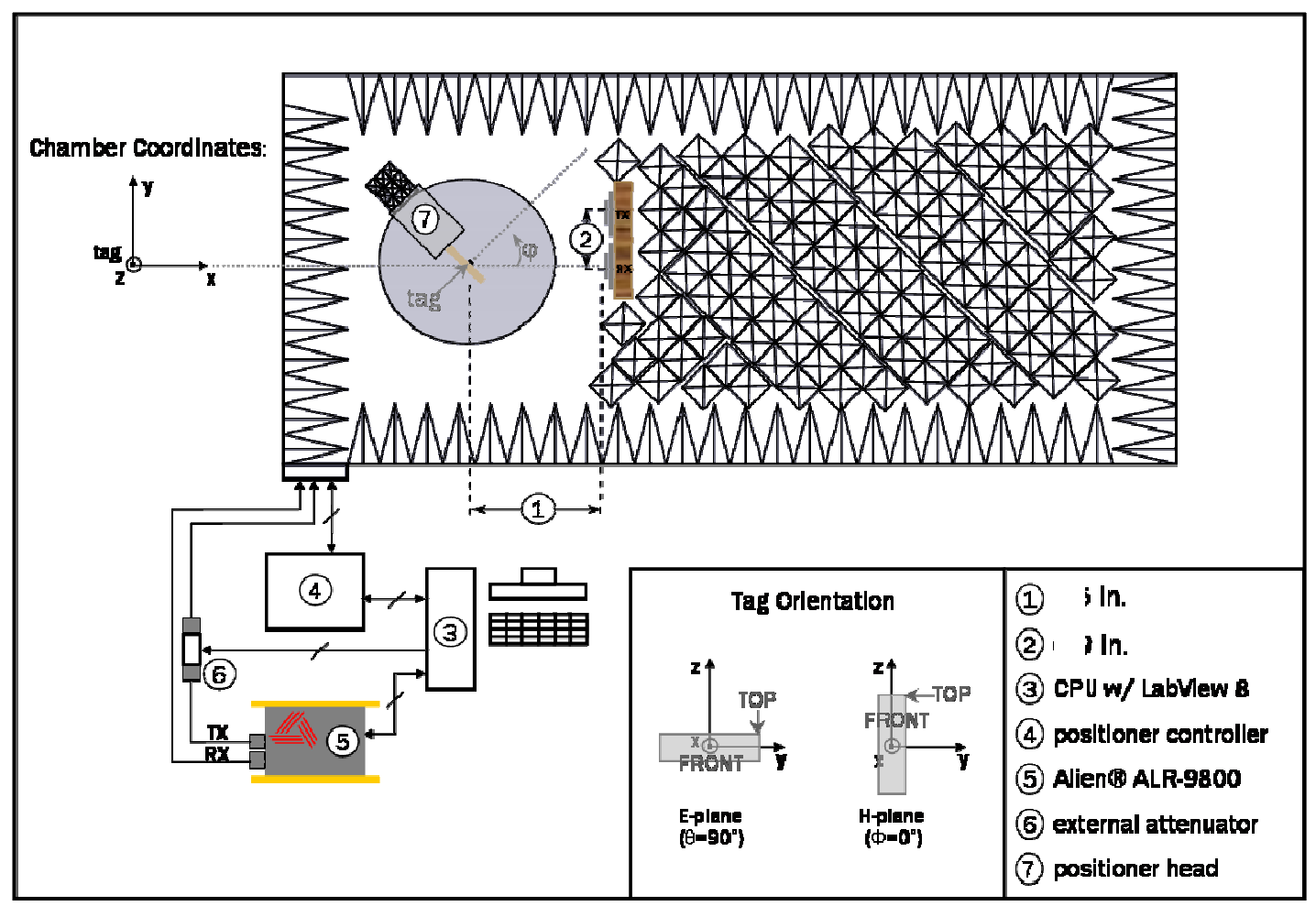

Figure 4.1: Original Chamber Configuration [42]

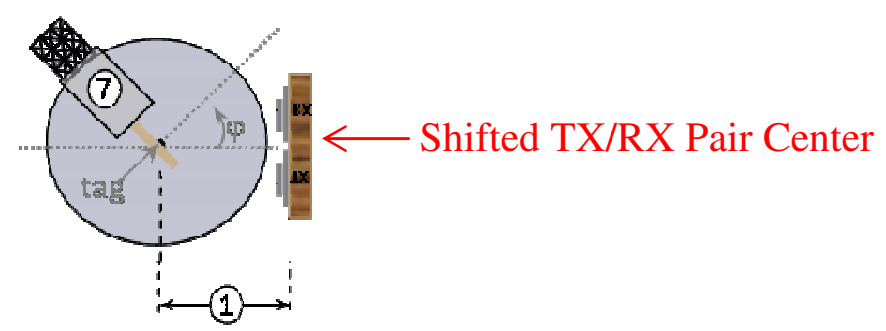

Figure 4.2: Modified Chamber Configuration (Tag Center to TX/RX Pair Center Alignment)

The RFID tag is mounted on the positioner to determine minimum transmit power as a function of angle in the E- and H-planes. The E-plane includes the propagating Efield lies, while the H-plane pertains to the propagating $\mathrm{H}$-field. The positioner and RFID reader are controlled by LabVIEW 8 software which also acquires data. 
Figure 4.3 illustrates the RFID tag fixture orientation for H-plane measurements while Figure 4.4 demonstrates the E-plane configuration. The pattern sweep was completed in two separate $180^{\circ}$ rotations to prevent positioner blockage between the TX/RX antennas and the RFID tag fixture. As recommended by [42], the test fixture was built using polystyrene to minimize electrical interference.

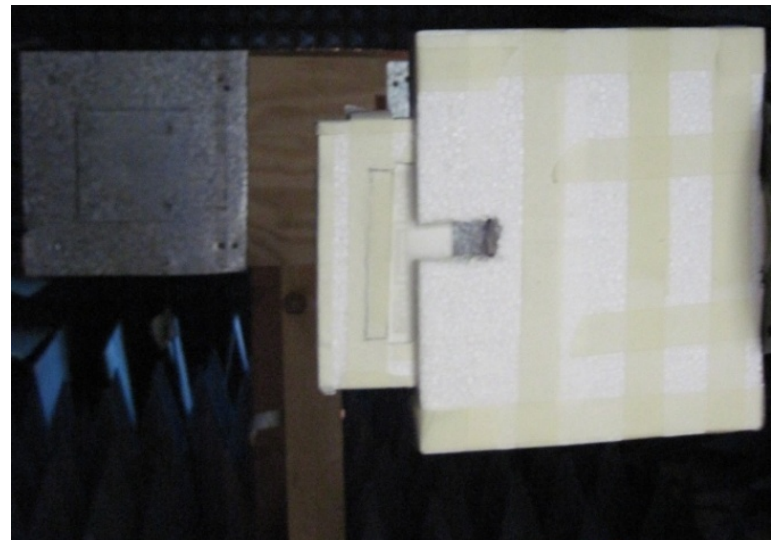

Figure 4.3: RFID Tag Fixture, H-Plane Co-pol

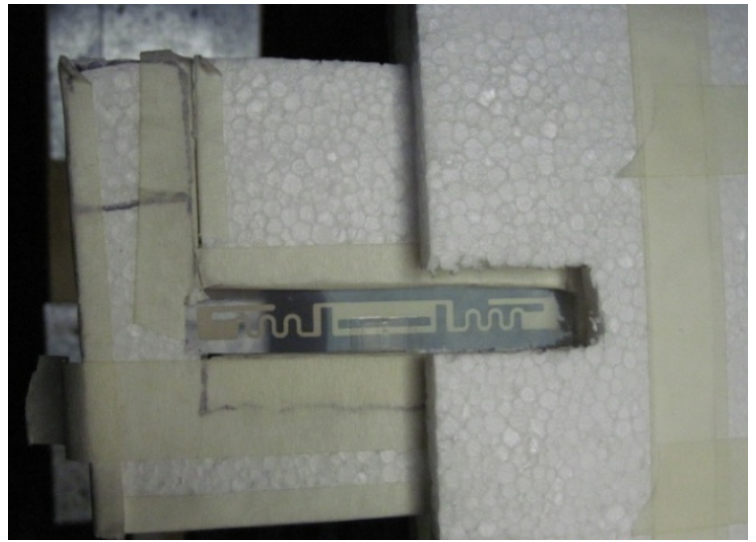

Figure 4.4: RFID Tag Fixture, E-Plane Co-pol

Four patterns are obtained for each tag: E-plane, co-pol and cross-pol; H-plane, co-pol and cross-pol. Test configurations (Table 4-1) are defined by the tag coordinate system [Figure 4.5 (a)] and the TX antenna polarization defined in Figure 4.5 (b). Detailed pattern acquisition procedures are given in [42].

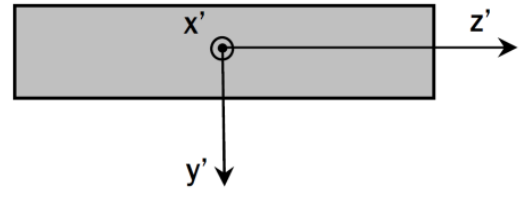

(a)

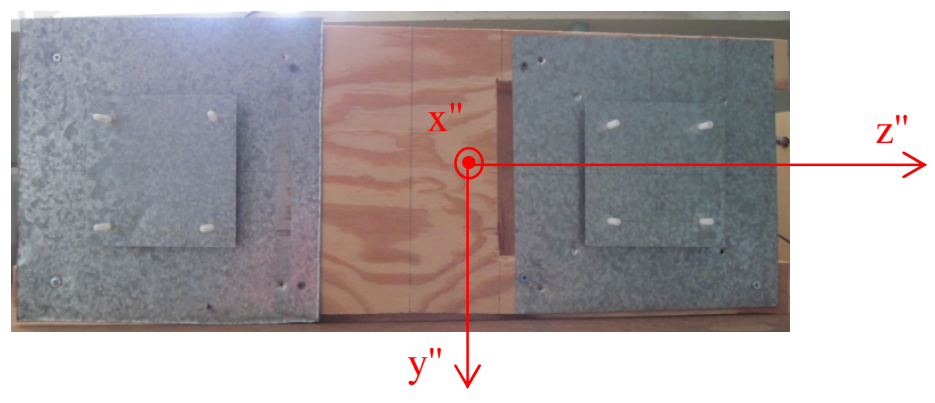

(b)

Figure 4.5: Test Configuration (a) Tag Coordinate System [42] (b) Patch Antenna Coordinate System 
Table 4-1: Tag and Patch Antenna Alignment: Chamber (unprimed), Tag (primed) and Patch Antenna (double-primed) Coordination Systems [42]

\begin{tabular}{|c|c|c|c|}
\hline Tag Alignment & $\begin{array}{c}\text { Patch Antenna } \\
\text { Alignment }\end{array}$ & Scan Plane & Co- or Cross-pol \\
\hline $\mathrm{z}^{\prime}=\mathrm{y}, \mathrm{x}^{\prime}=\mathrm{x}$ & $\mathrm{z}^{\prime \prime}=-\mathrm{y}, \mathrm{y}^{\prime \prime}=-\mathrm{z}$ & $\mathrm{E}$ & Cross \\
\hline $\mathrm{y}^{\prime}=\mathrm{y}, \mathrm{x}^{\prime}=\mathrm{x}$ & $\mathrm{z}^{\prime \prime}=-\mathrm{y}, \mathrm{y}^{\prime \prime}=-\mathrm{z}$ & $\mathrm{H}$ & Co \\
\hline $\mathrm{z}^{\prime}=\mathrm{y}, \mathrm{x}^{\prime}=\mathrm{x}$ & $\mathrm{z}^{\prime \prime}=-\mathrm{z}, \mathrm{y}^{\prime \prime}=\mathrm{y}$ & $\mathrm{E}$ & Co \\
\hline $\mathrm{y}^{\prime}=\mathrm{y}, \mathrm{x}^{\prime}=\mathrm{x}$ & $\mathrm{z}^{\prime \prime}=-\mathrm{z}, \mathrm{y}^{\prime \prime}=\mathrm{y}$ & $\mathrm{H}$ & Cross \\
\hline
\end{tabular}

Table 4-2 indicates the relationship between actual transmitted RF Power (dBm) and reader attenuation $(\mathrm{dB})$ [42]. This conversion is necessary for read range calculations.

\begin{tabular}{|cc|}
\hline \multicolumn{2}{|c|}{ Measured } \\
\hline $\begin{array}{c}\text { Attenuation } \\
\text { (dB) }\end{array}$ & $\begin{array}{c}\text { RF Power } \\
\text { (dBm) }\end{array}$ \\
\hline 0.0 & 31.9 \\
0.9 & 31.0 \\
1.9 & 30.0 \\
2.6 & 29.3 \\
3.4 & 28.5 \\
4.3 & 27.6 \\
5.6 & 26.3 \\
6.4 & 25.5 \\
7.3 & 24.6 \\
8.5 & 23.4 \\
9.0 & 22.9 \\
9.6 & 22.3 \\
10.7 & 21.2 \\
11.6 & 20.3 \\
12.3 & 19.6 \\
12.8 & 19.1 \\
\hline
\end{tabular}

Table 4-2: Attenuation vs. Measured RF Power, ALR-9800 RFID Reader [42]

\subsection{Fabrication Method Selection}

To verify the the fabrication and material selection process, four prototype RFID tags with Sirit tag pattern RSI-674 [27] were tested to confirm operation. Four different configurations (Tags \#2 through \#4) and a commercial Sirit tag, summarized in Table 4-3, were characterized in the chamber. Tag performance was compared to determine the 
preferred fabrication process (conductive vs. Scotch tape and laminated vs. nonlaminated).

Table 4-3: Fabricated Tag Configuration

\begin{tabular}{|c|c|c|}
\hline Tag & Lamination & IC Attachment Method \\
\hline 1 & N/A. Sirit,Commercial Tag & \\
\hline 2 & No & Conductive tape \\
\hline 3 & No & Scotch tape \\
\hline 4 & Yes & Conductive tape \\
\hline 5 & Yes & Scotch tape \\
\hline
\end{tabular}

Figure 4.7 shows the commercial Sirit tag characterization. The radiation pattern indicates a shift due to tag positioning relative to the TX and RX antenna centers (see Figure 4.6). The distance between the TX and RX antennas is $19 \mathrm{in}(48.26 \mathrm{~cm})$ and from the TX or RX antennas to the tag is 39in $(99.06 \mathrm{~cm})$. The angular relationship is shown below.

$$
\theta=\tan ^{-1}\left(\frac{24.13}{99.06}\right)=13.69^{\circ}
$$

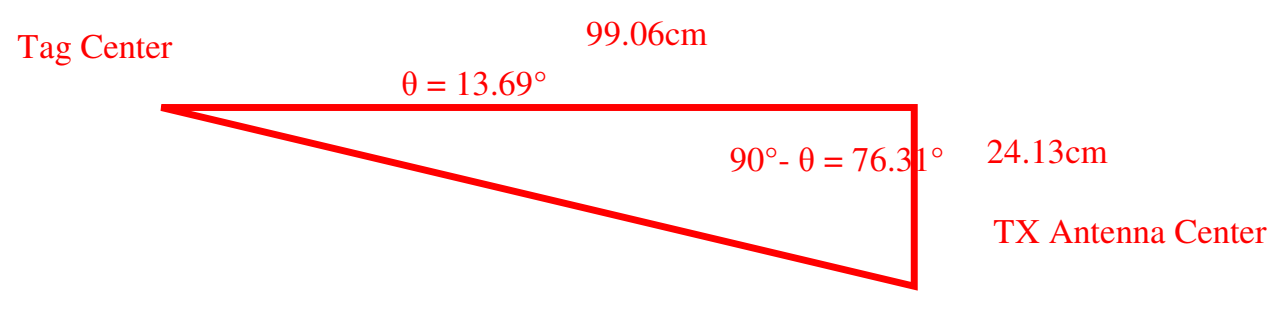

Figure 4.6: Angular Relationship, TX or RX Antenna and Tag Centers 

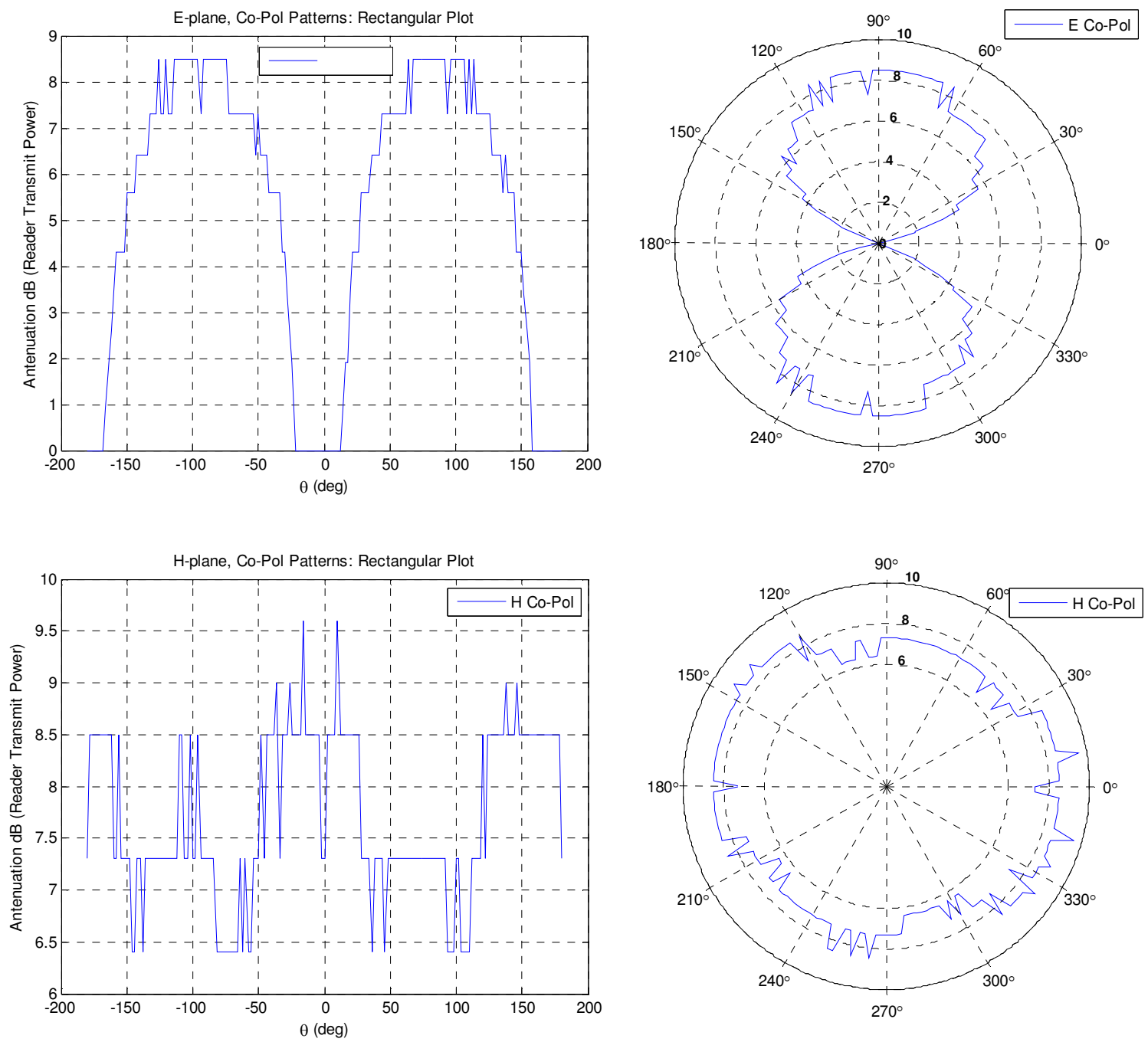

Figure 4.7: Sirit Tag E- and H-Plane Patterns, E- and H-Plane X-pol: OdB (Not Plotted)

Figure 4.8 shows E-plane and H-plane co-pol patterns for Tags \#2 and \#3 and shows comparable performance for conductive vs. Scotch tape. The final fabrication uses conductive tape due to increased durability over Scotch tape. Similarly, Figure 4.9 compares performance between laminated and non-laminated tags. Performance is identical in the E-plane, but degraded between $-90^{\circ}$ and $90^{\circ}$ in the H-plane. Overall, lamination does not prevent tag operation; it is acceptable for use in the final fabrication. 

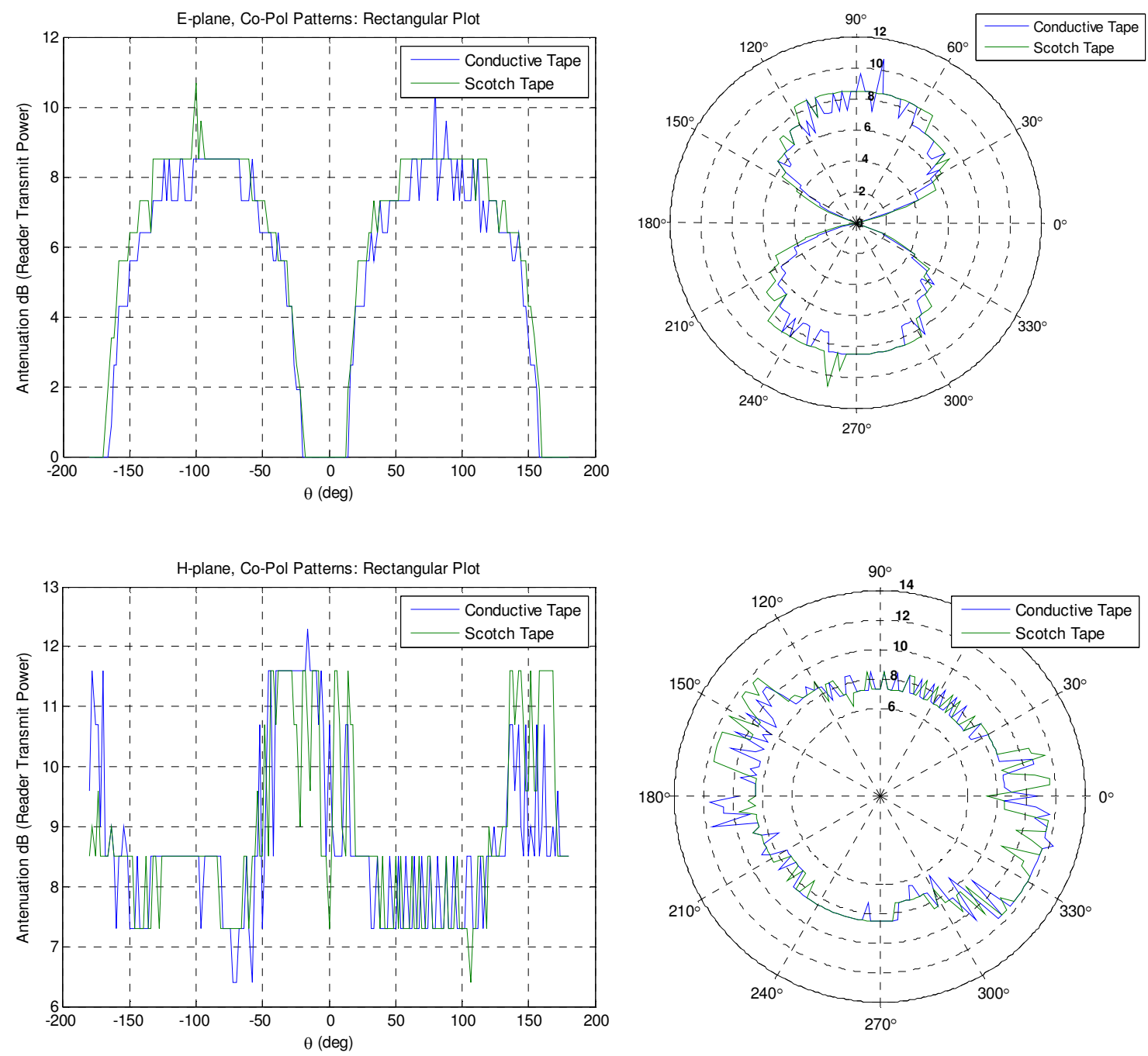

Figure 4.8: E- and H-Plane Pattern Comparison (Conductive vs. Scotch Tape) 

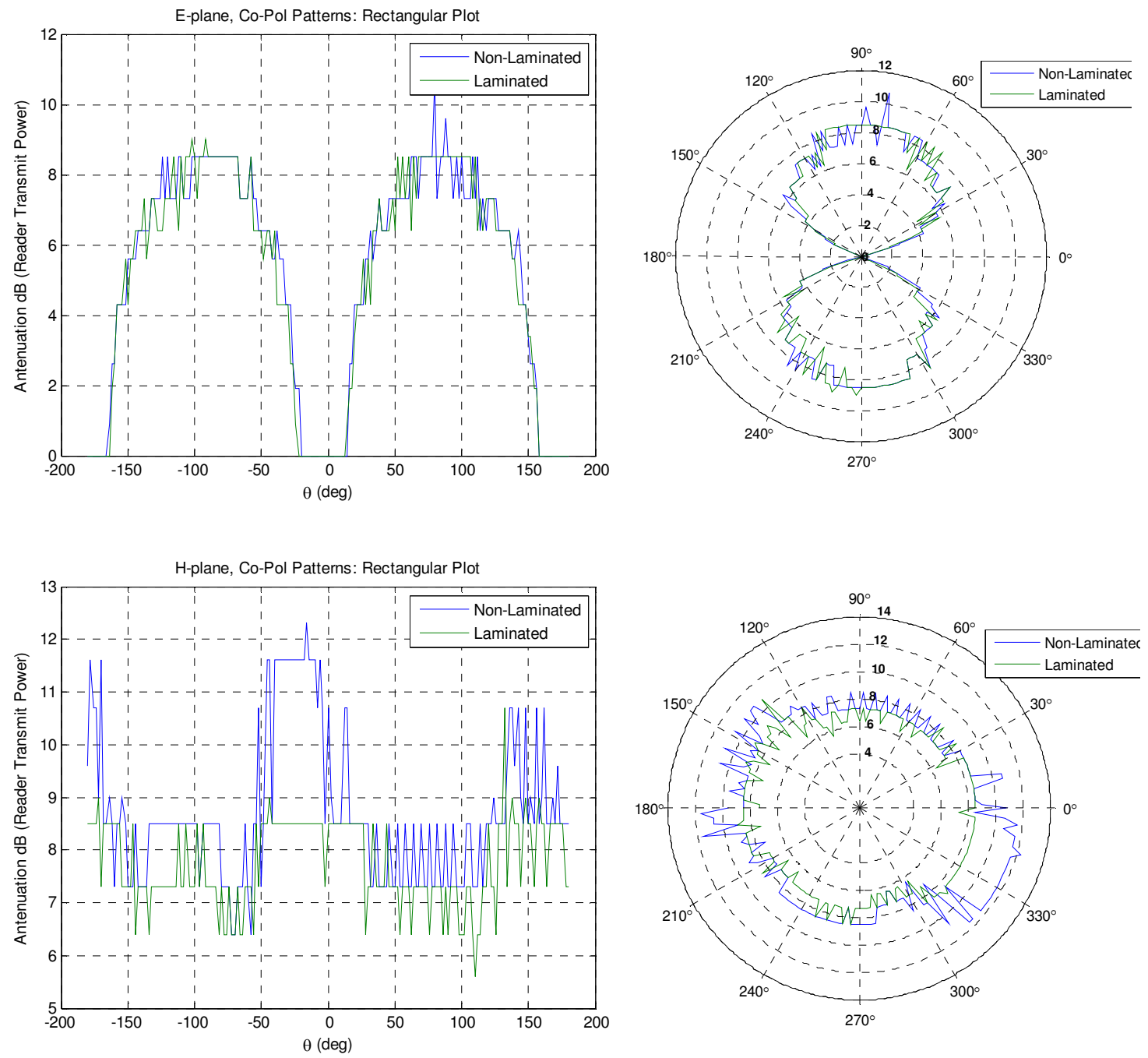

Figure 4.9: E- and H-Plane Pattern Comparison (Laminated vs. Non-Laminated)

\subsection{RFID Tag Design E- and H-Plane Patterns}

The three RFID tags were fabricated and tested using the chamber setup as described in Section 4.1. E- and H-plane patterns are shown in Figure 4.10 through Figure 4.12 for tag Designs \#1through \#3, respectively. The E- and H-Plane cross pol patterns for all three designs have $0 \mathrm{~dB}$ values and were hence not plotted.

To facilitate comparisons, the three plots are superimposed with the Sirit tag's pattern from Section 4.2 in Figure 4.13. 

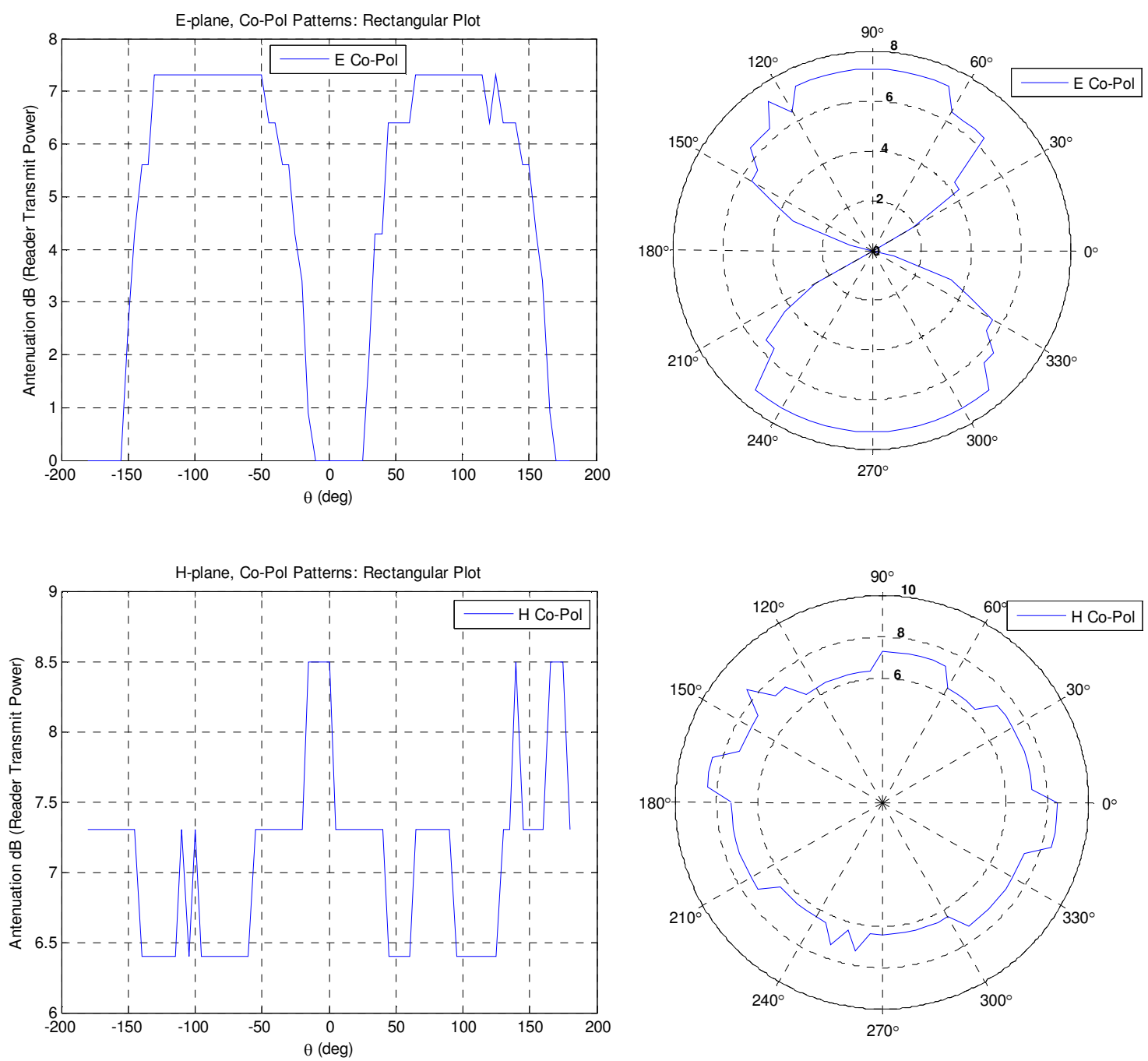

Figure 4.10: Tag Design \#1 E- and H-Plane Patterns, E- and H-Plane X-pol: OdB (Not Plotted) 

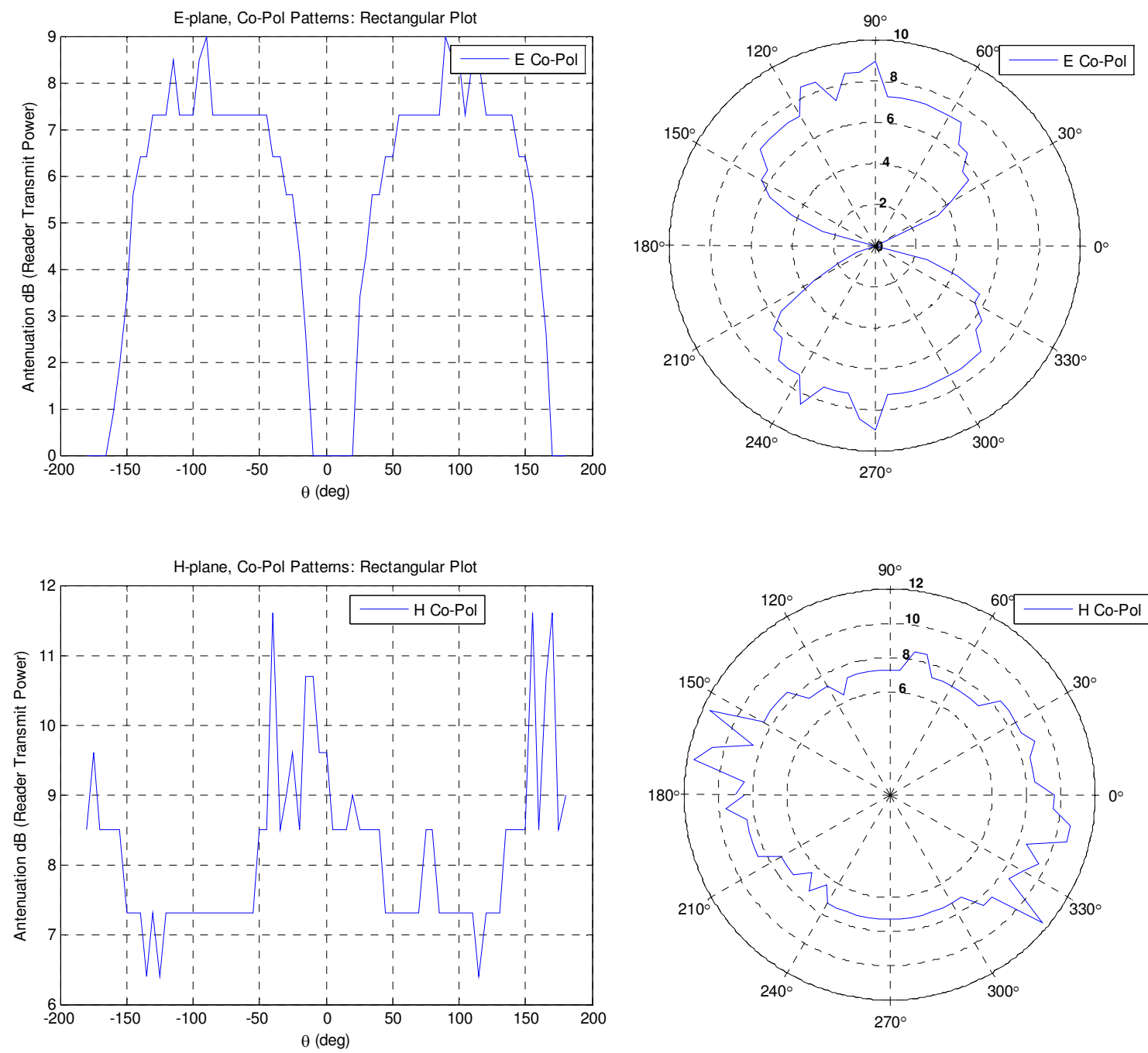

Figure 4.11: Tag Design \#2 E- and H-Plane Patterns, E- and H-Plane X-pol: OdB (Not Plotted) 

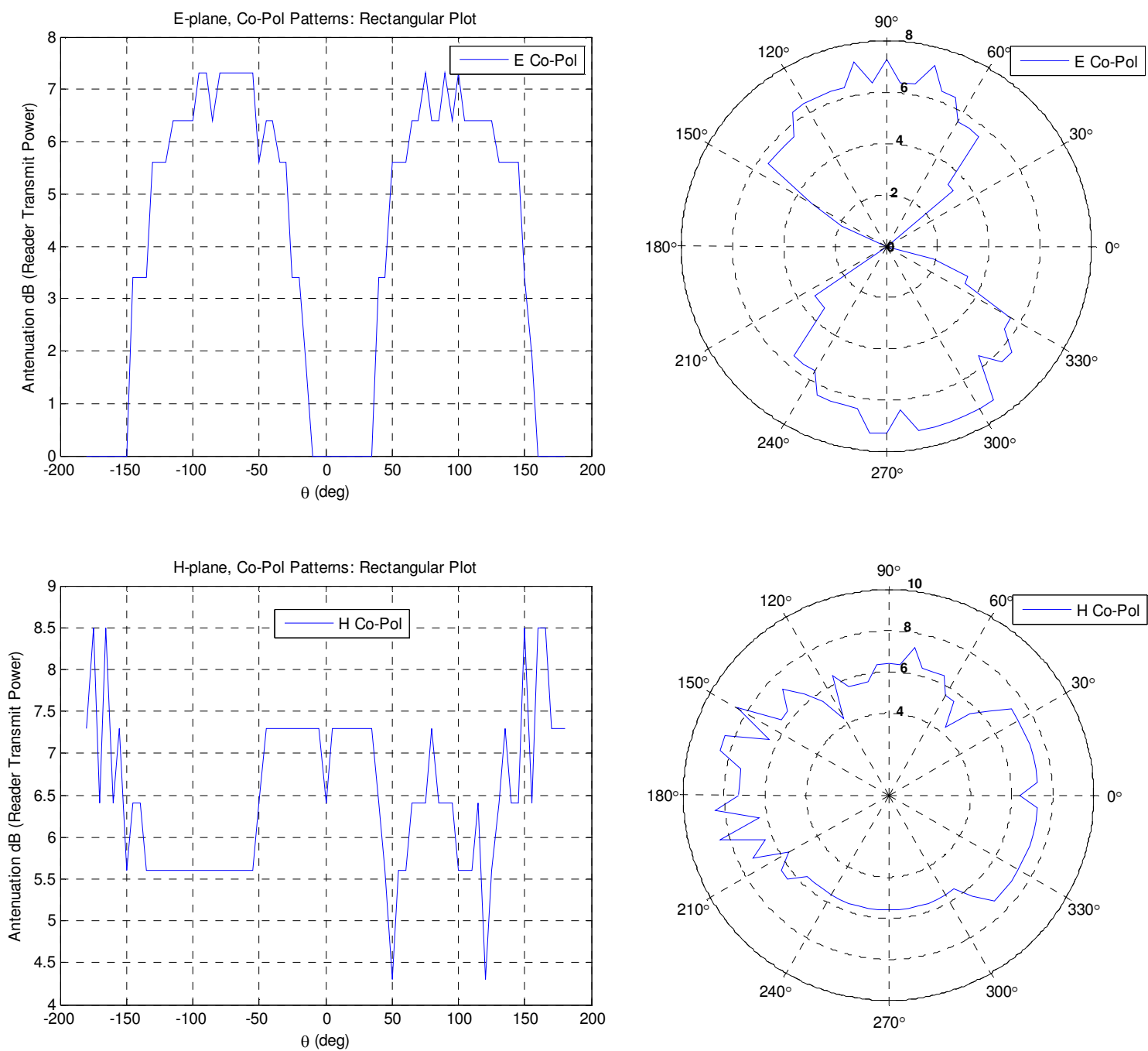

Figure 4.12: Tag Design \#3 E- and H-Plane Patterns, E- and H-Plane X-pol: OdB (Not Plotted) 

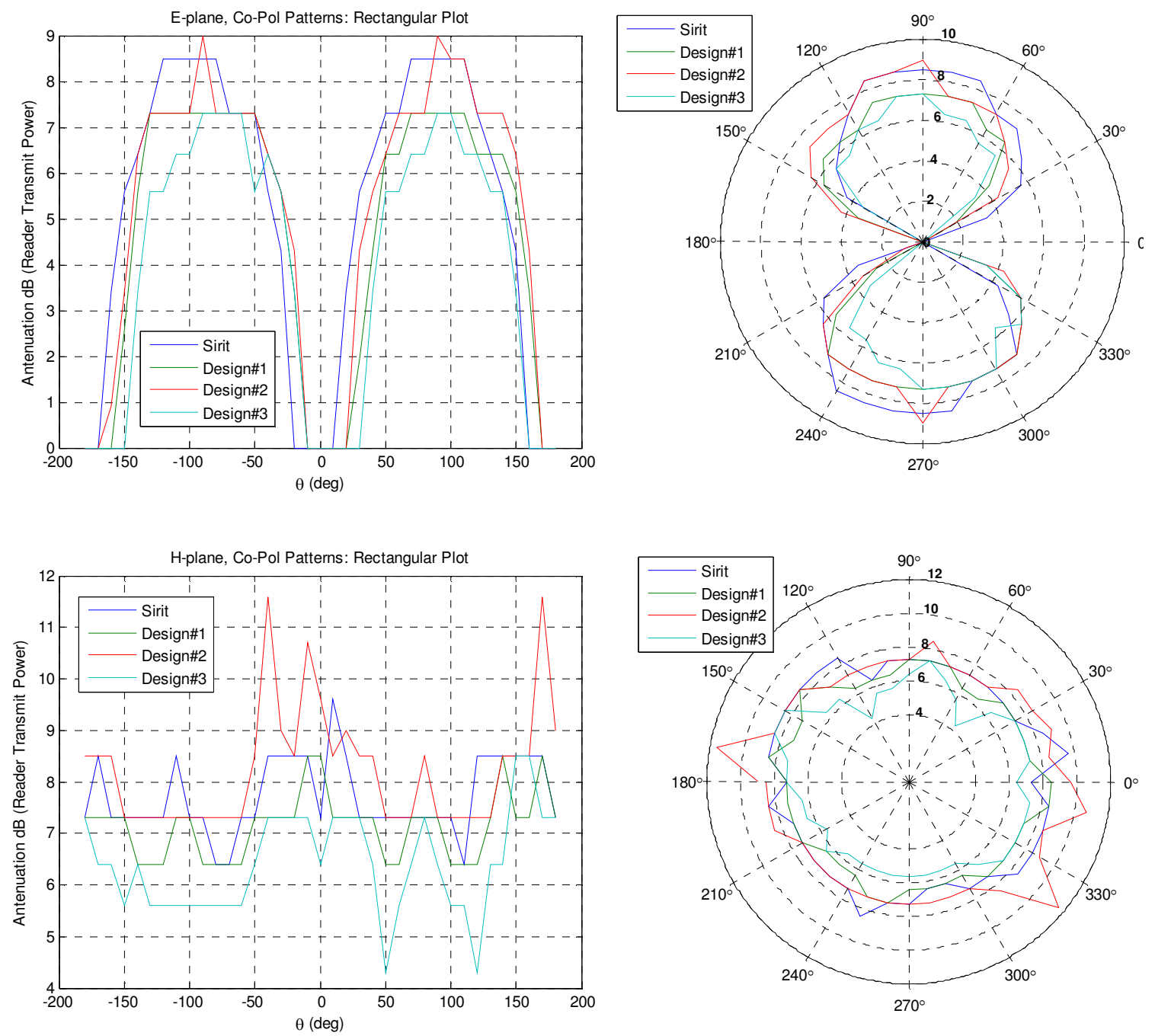

Figure 4.13: E- and H-Plane Pattern Comparison

According to Figure 4.13, tag Design \#2 results exceed the commercial design response by up to $2 \mathrm{~dB}$ in the $\mathrm{H}$-plane.

\subsection{Patch Antenna Gain Extraction}

The read range calculation requires transmit antenna gain. The TX and RX patch antenna pair is shown in Figure 4.14. To obtain antenna gain, both patch antennas' exact dimensions are used to create an EMPro simulation model; gain and $\mathrm{S}_{11}$ responses are calculated. 


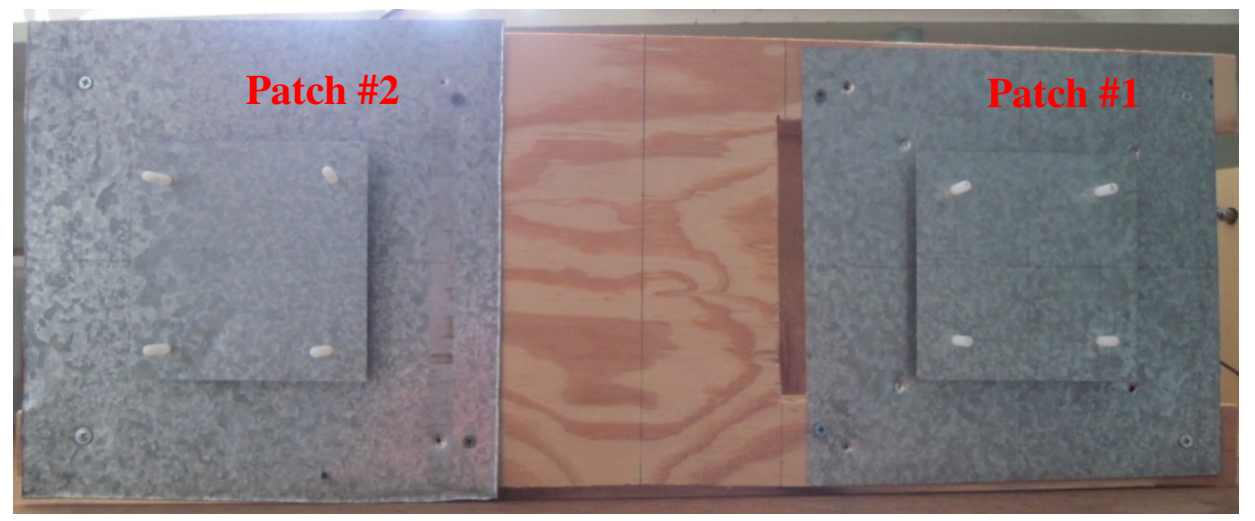

Figure 4.14: TX/RX Antenna Pair

Figure 4.15 shows EMPro models. Patch \#1 has a maximum gain of $9.75 \mathrm{dBi}$

while Patch \#2 has 9.97dBi. Both patch antennas have identical radiation patterns.

According to the IS11I plot (Figure 4.16), both patch antennas exhibit approximately

$-16 \mathrm{~dB}$ at $920 \mathrm{MHz}$, acceptable for RFID reader operations as bandwidth is defined by the $-10 \mathrm{~dB}$ threshold.
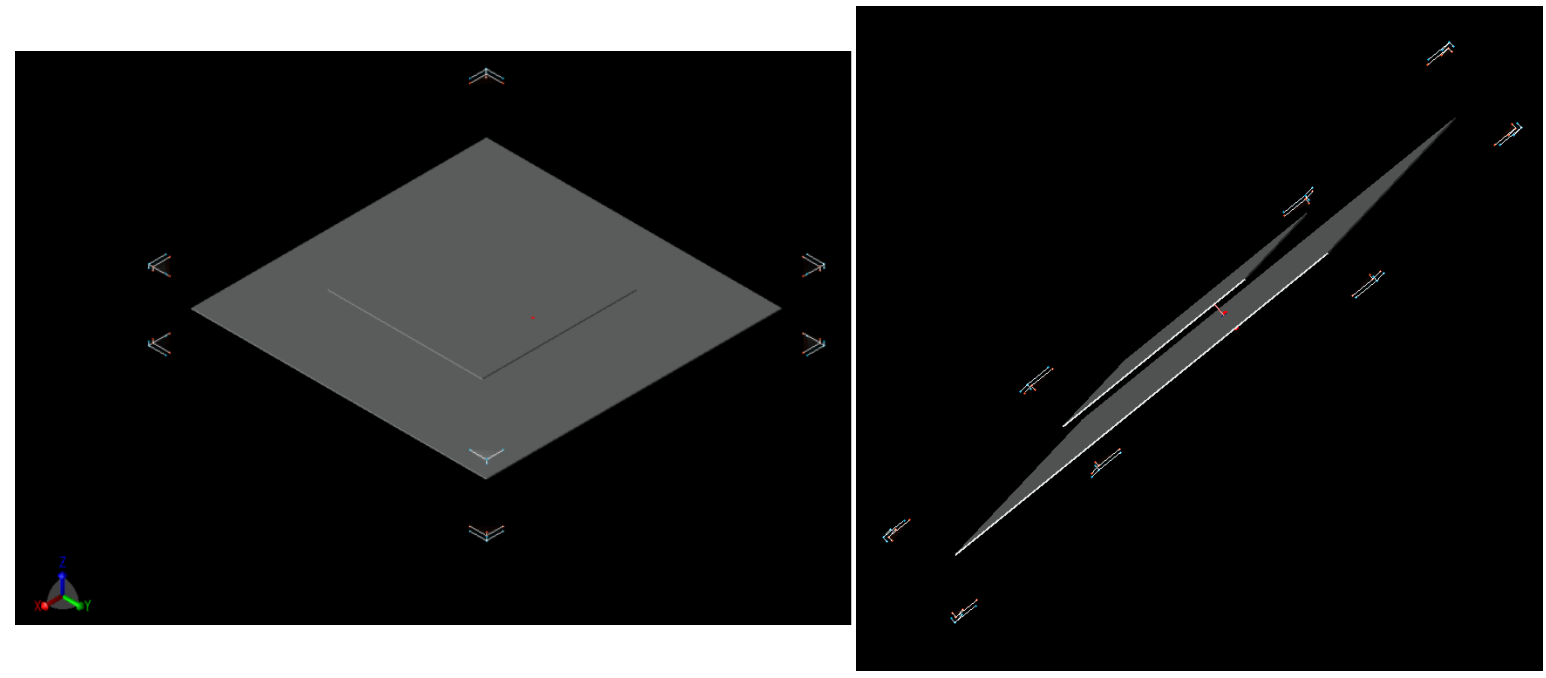

(a)

(b)

Figure 4.15: EMPro TX/RX Patch Antenna Models (a) Patch \#1 (b) Patch \#2 (E-plane: YZ) 


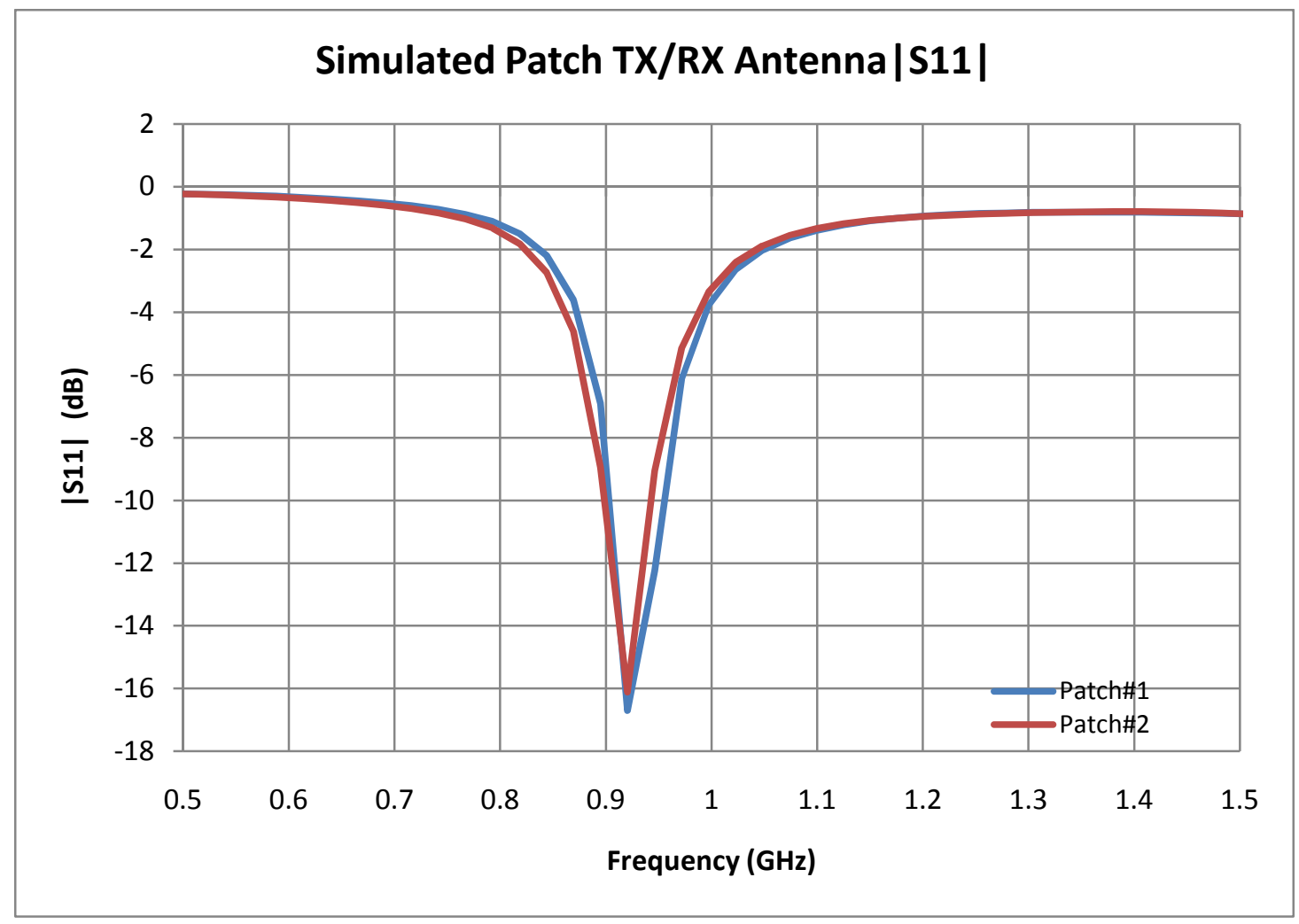

Figure 4.16: Simulated Patch TX/RX Antenna $\left|S_{11}\right|$ vs. Frequency

According to the simulation model's patch orientation, the $\mathrm{YZ}$ plane $\left(\varphi=90^{\circ}\right)$ is the E-plane while the $\mathrm{XZ}$ plane $\left(\varphi=0^{\circ}\right)$ is the H-plane.

E-plane co-pol $\left(\varphi=90^{\circ}\right)$ simulated pattern yield gain for the read range calculation, see Figure 4.17. Maximum gain for both patch antennas is approximately 10dBi. Rectangular plots are shown in Figure 4.18 to allow gain values determination at specific angles to facilitate read range calculations. 


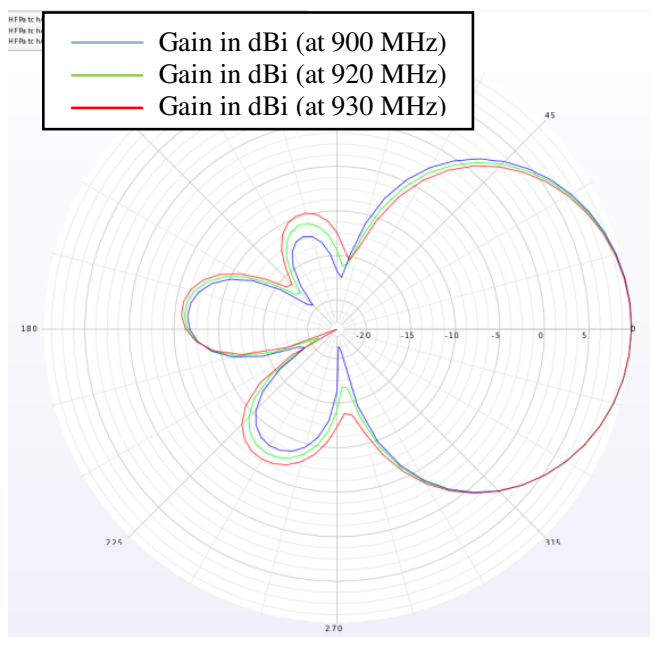

(a)

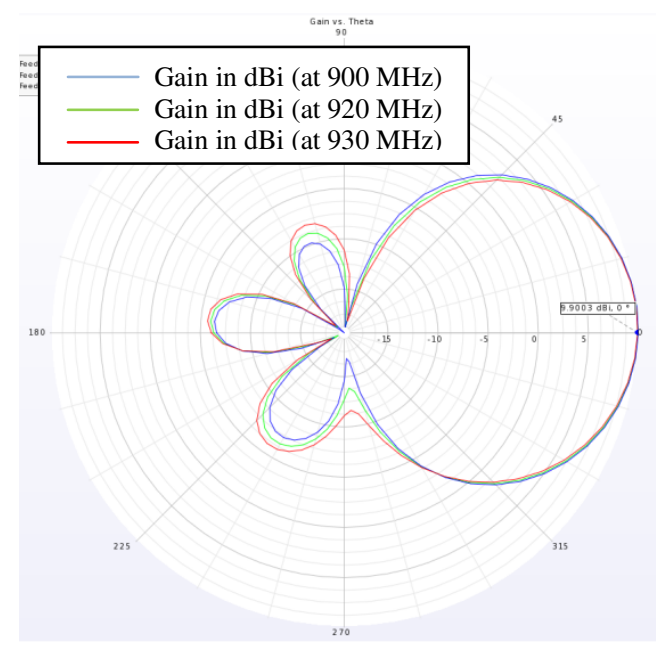

(b)

Figure 4.17: Radiation Pattern Phi Cut $\left(\varphi=9^{\circ}\right)$ E-Plane Co-Pol (a) Patch \#1 (b) Patch \#2

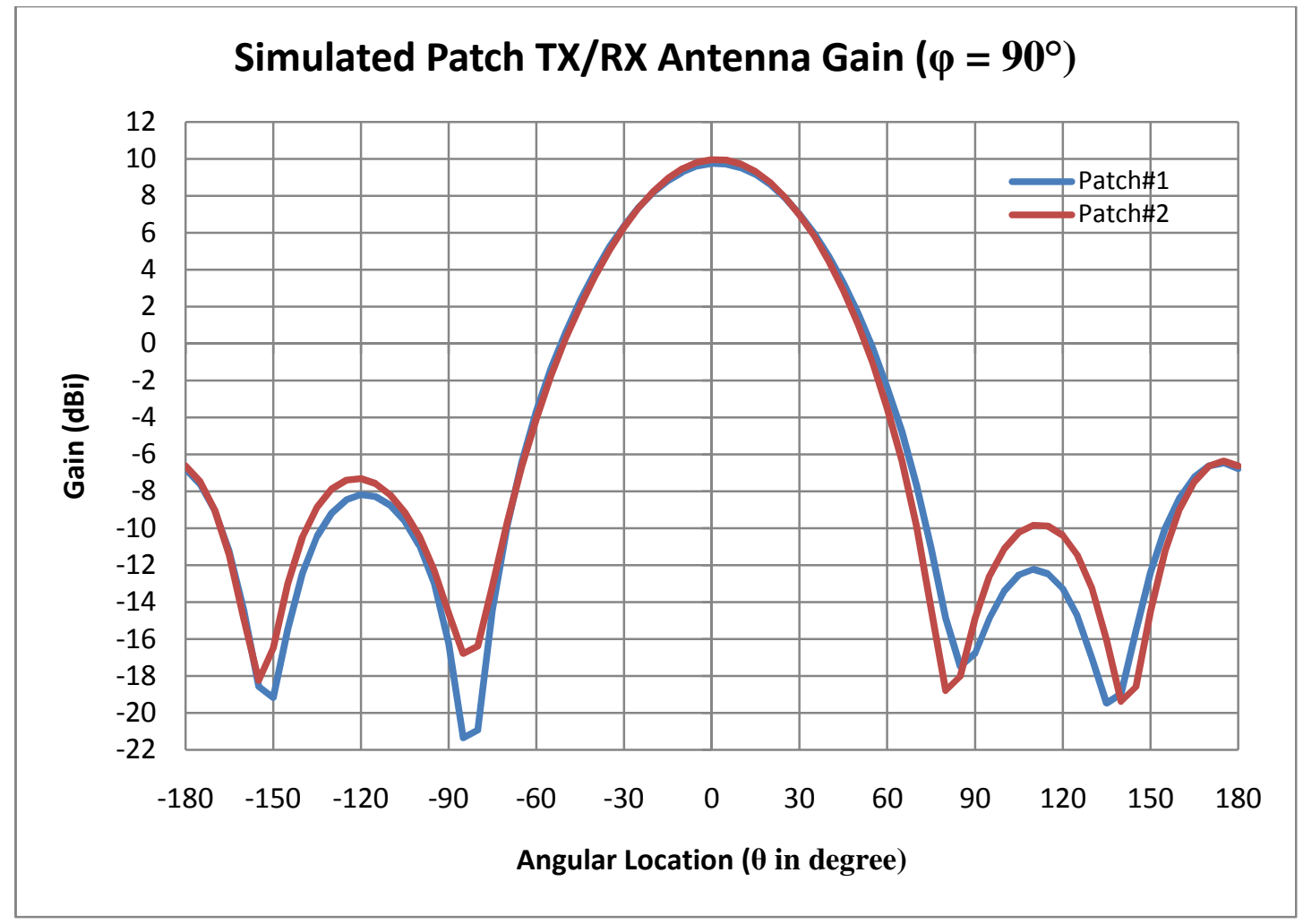

Figure 4.18: Radiation Pattern Phi Cut $\left(\varphi=9^{\circ}\right)$ Rectangular Plot 


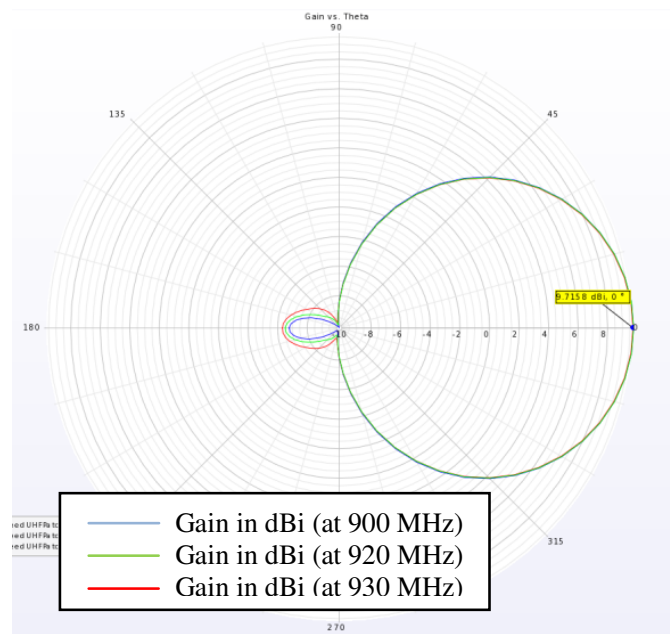

(a)

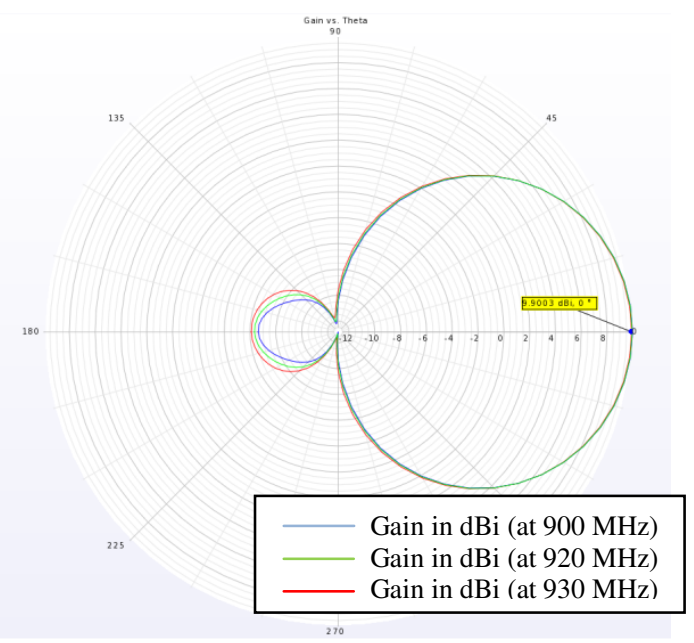

(b)

Figure 4.19: Radiation Pattern Phi Cut $\left(\varphi=0^{\circ}\right)$ H-Plane Co-Pol (a) Patch \#1! (b) Patch \#2

Based on Figure 4.6 calculations, the relative angular location between the TX antenna and tag centers (13.69o) is indicated in Figure 4.20, which yields a transmit gain of approximately $9 \mathrm{dBi}$.

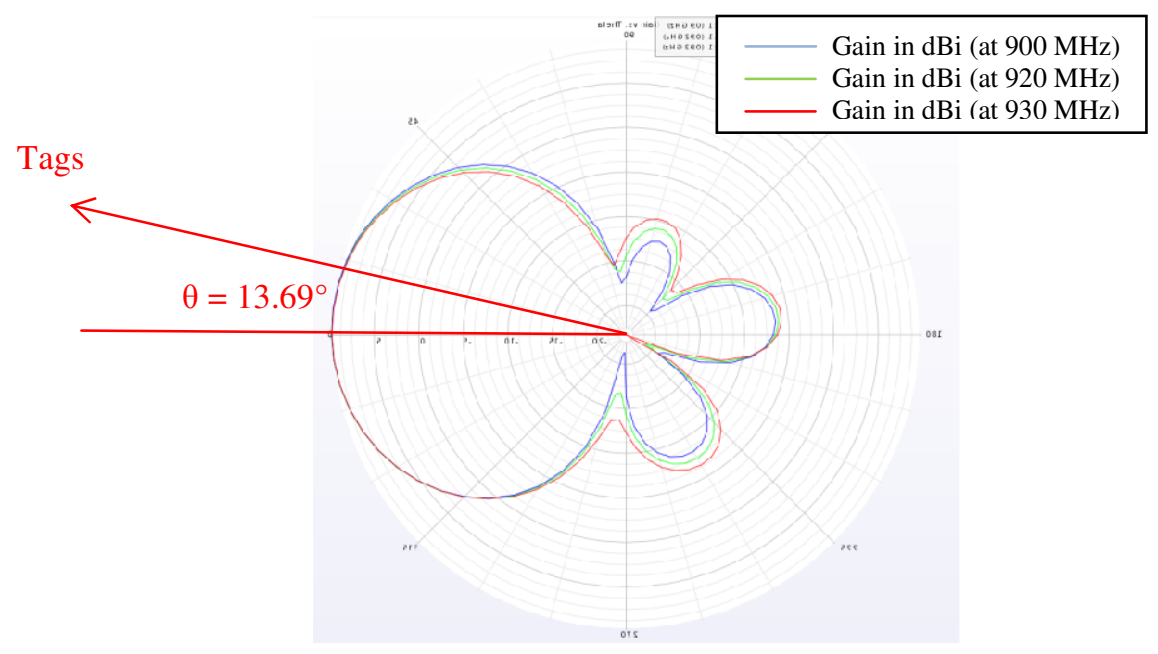

Figure 4.20: Angular Relationship, TX Antenna and Tag 


\subsection{RFID Read Range Calculations}

Using 4W maximum EIRP, 9dBi antenna gain and 0.99m antenna-tag distance, read range is calculated for each reader attenuation value (Table 4-2) according to equation (4.4), see Section 4.1. Based on these values, read range polar patterns are plotted for the three tag designs and the Sirit tag. Since read range is measured in a controlled environment (anechoic chamber), calculated values represent the ideal case; typical tag operating environments decrease the effective read range.

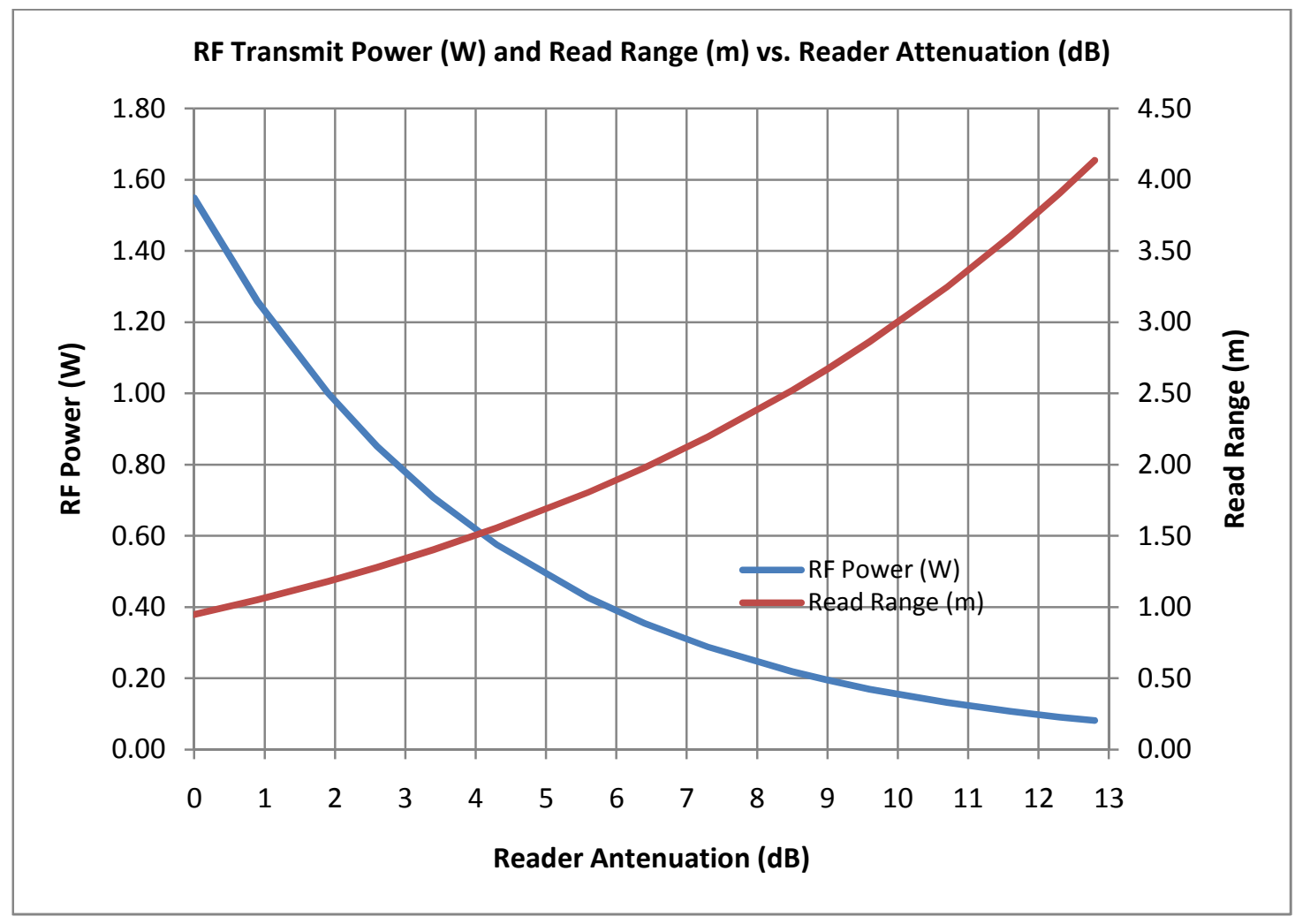

Figure 4.21: RF Transmit Power (W) and Read Range (m) vs. Reader Attenuation (dB) 


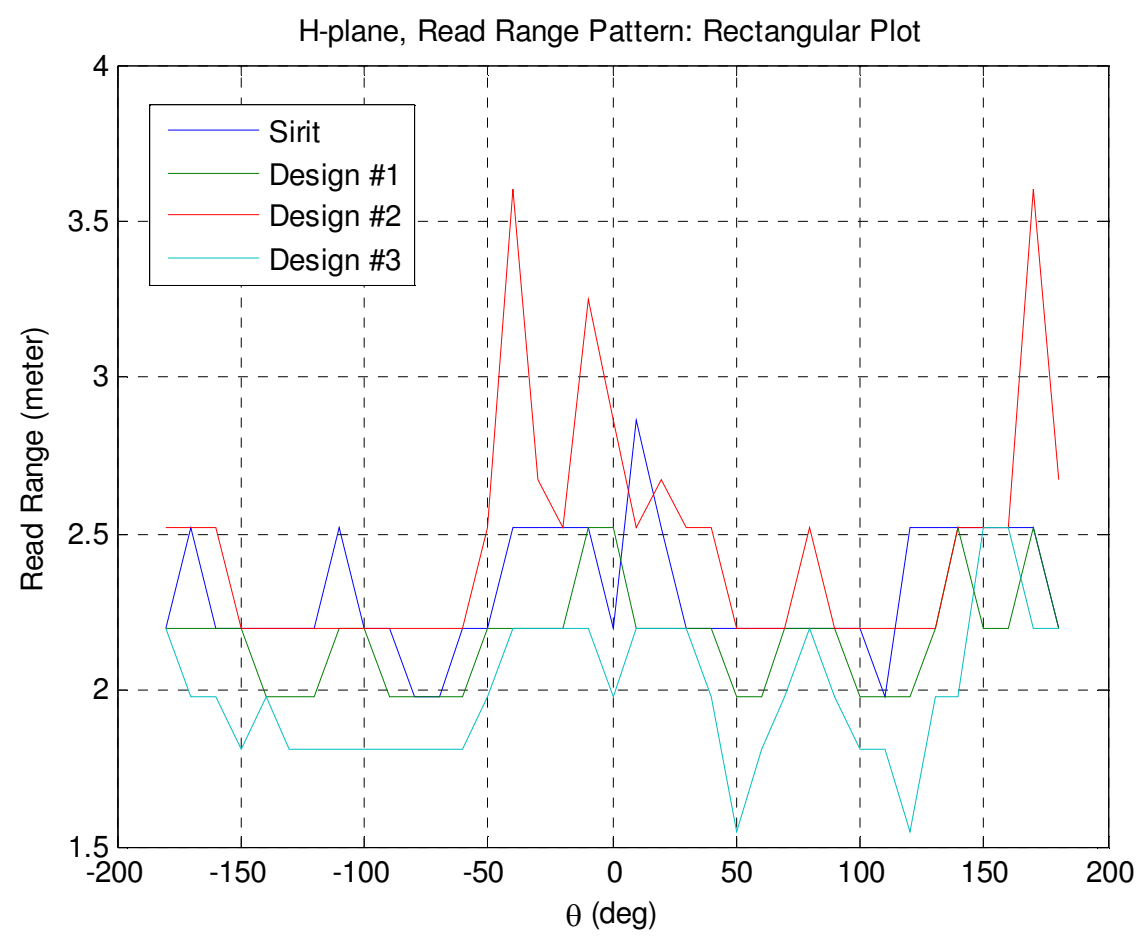

(a)

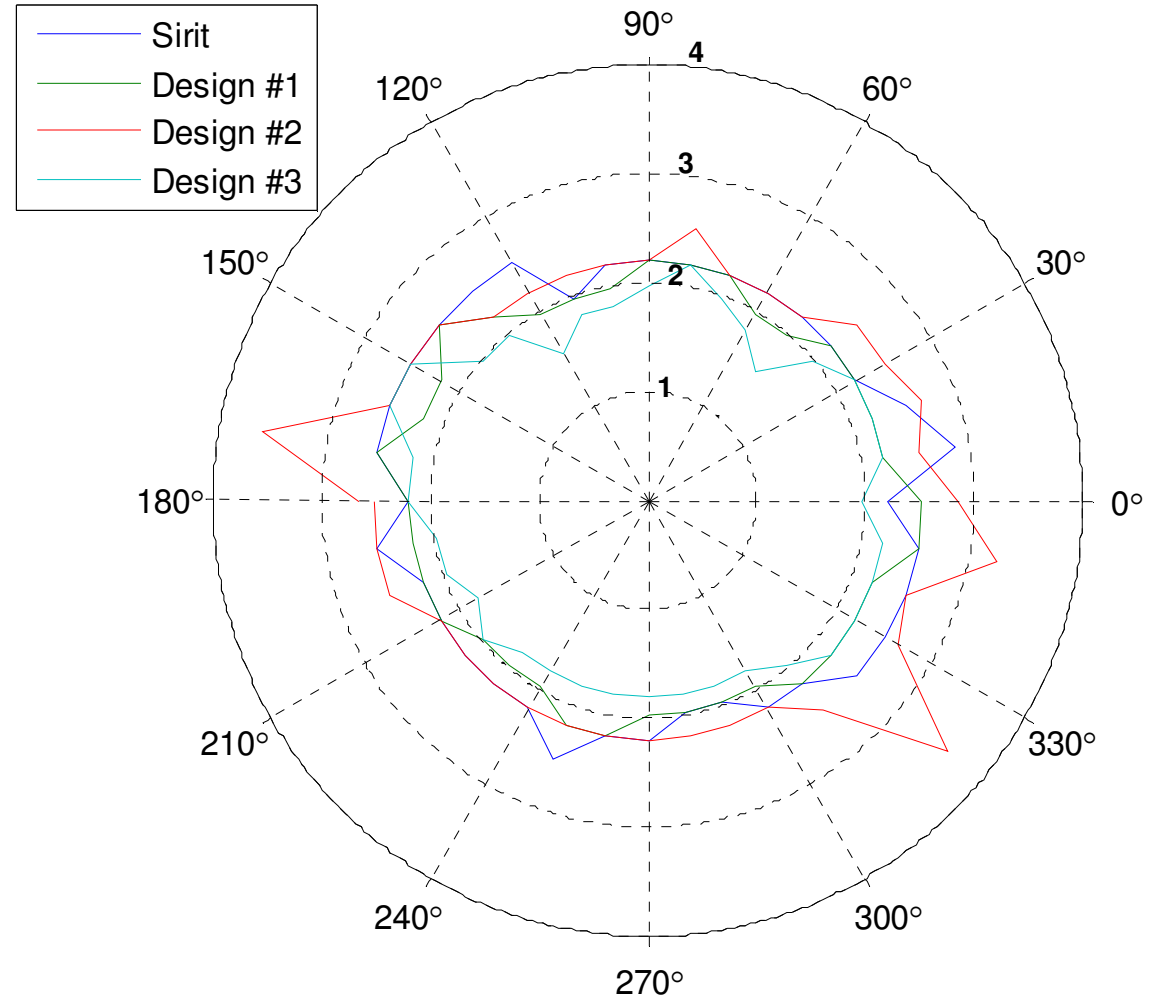

(b)

Figure 4.22: RFID Tag Read Range (a) Rectangular Plot (b) Read Range Pattern 
Tag Design \#2 produced the maximum read range; approximately $3.5 \mathrm{~m}$. It also outperforms (up to $3 \mathrm{~m}$ greater) the commercial tag between $-90^{\circ}$ and $90^{\circ}$ while the (Designs \#1 and \#3) do not perform as well as the commercial tag. Read ranges are $1 \mathrm{~m}$ shorter at most angles. 


\section{CONCLUSIONS AND FUTURE RECOMMENDATIONS}

This project produced the first ever Cal Poly constructed UHF passive RFID tag. Processes were established to simulate, design, fabricate, and test RFID tags to operate with the Monza 3 RFID IC [12]. One of the RFID tag prototypes exceeded the read range performance of a commercial Sirit tag [23].

Using IC-substrate packages (strap) supplied by Sirit Inc [23], this project focused on impedance matching the tag antenna to the RFID IC (Monza 3). The RFIC input impedance was measured using a vector network analyzer (VNA) and a transistor test fixture while the Sirit tag was modeled in simulation software package EMPro to measure antenna impedance. Accurate IC impedance values were not available due to IC probe unavailability. The Sirit tag model simulated impedance provided an alternate method of approximating the IC impedance. For maximum IC-tag antenna power transfer, the tag antenna is complex conjugate matched to the Monza 3 IC.

Three tag antenna designs based on the Monza 3 datasheet, measurements [22], and EMPro simulation were developed, simulated and impedance matched to the IC using EMPro. To verify impedance matching, the three tag designs were fabricated on FR4 substrates with copper traces to facilitate antenna input impedance measurements through differential probe methods. The impedance measurements require VNA port extensions to translate the measurement plane to the tag antenna. However, measured and simulated impedance values are not in agreement (relatively constant offset). Additional experiments are required to determine the cause.

Through the Cal Poly Graphic Communication Department, the three tag antenna designs were silver conductive ink screen-printed onto 8 mil thick PET substrates [13]. 
Strap package to antenna attachments were established manually using double-sided conductive tape.

Finally, the fabricated tags were functionally verified through read range measurements in the Cal Poly Anechoic Chamber. These results indicate that one of the tag designs has a read range up to $3 \mathrm{~m}$ greater than the Sirit commercial tag.

Operational UHF passive RFID tags were successfully produced at Cal Poly and the fabrication process is documented in this thesis. The tag designs had similar or even improved (tag design \#2) read range performance compared to the Sirit commercial tag. Moreover, the designed tag's conductive area was reduced relative to the Sirit tag.

Below is a list of future recommendations

1. In this project, RFID tag antenna designs were optimized for best impedance matching. Other parameters, i.e.: tag size and bandwidth, could also be examined.

2. Determine reasons for measured vs. simulated offsets in the RFID impedance measurements using the differential probe method.

To illustrate differences between measurements taken inside and outside the anechoic chamber, measured impedance responses are plotted in Figure 5.1 and Figure 5.2. The measurement environment has minimal impact $( \pm 2 \Omega)$ for resistance while a $\pm 10 \Omega$ offset is observed in the reactance measurement. This area requires further investigation. 


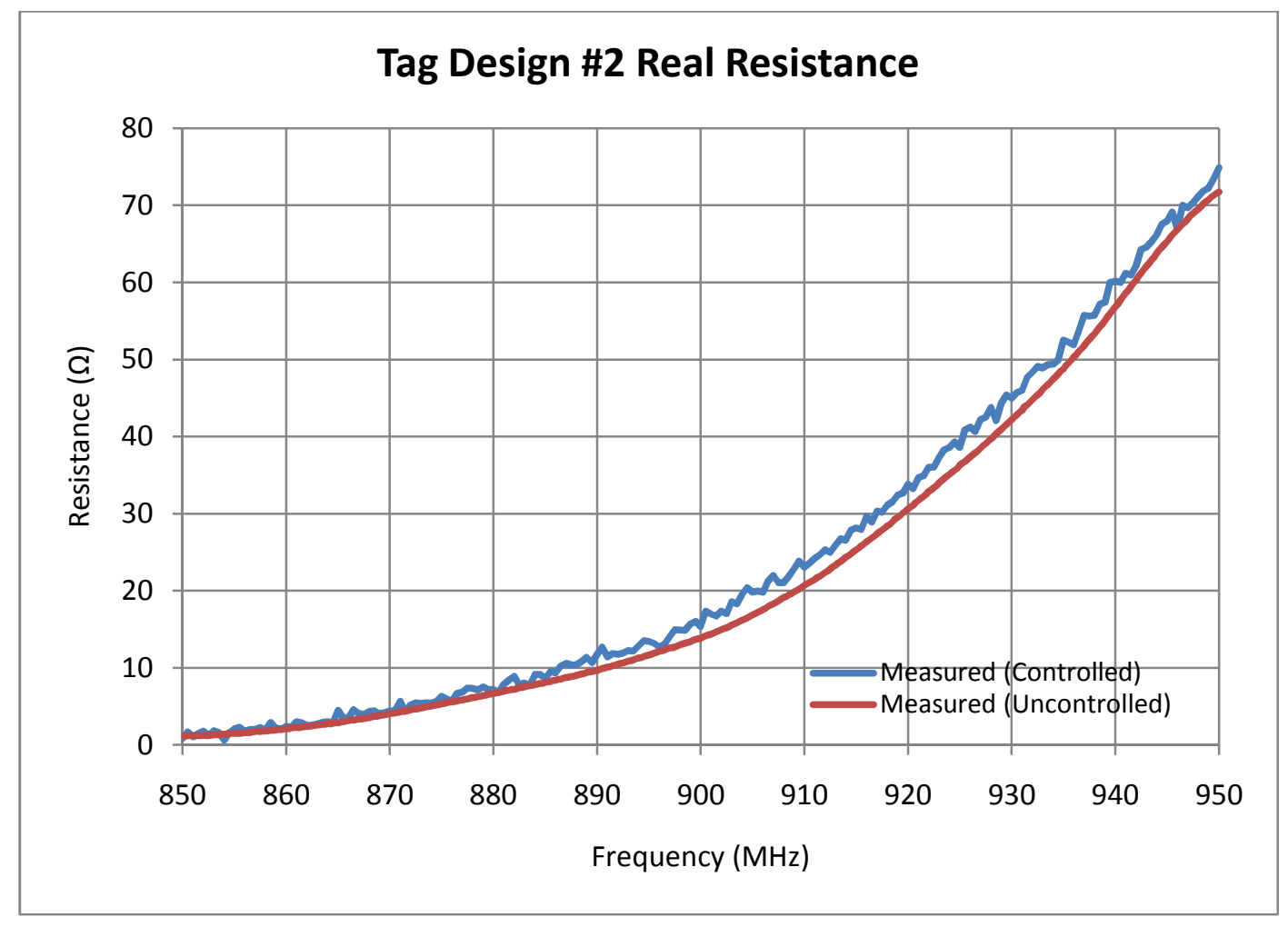

Figure 5.1: Measured RFID Antenna Resistance (Controlled vs. Uncontrolled Environment)

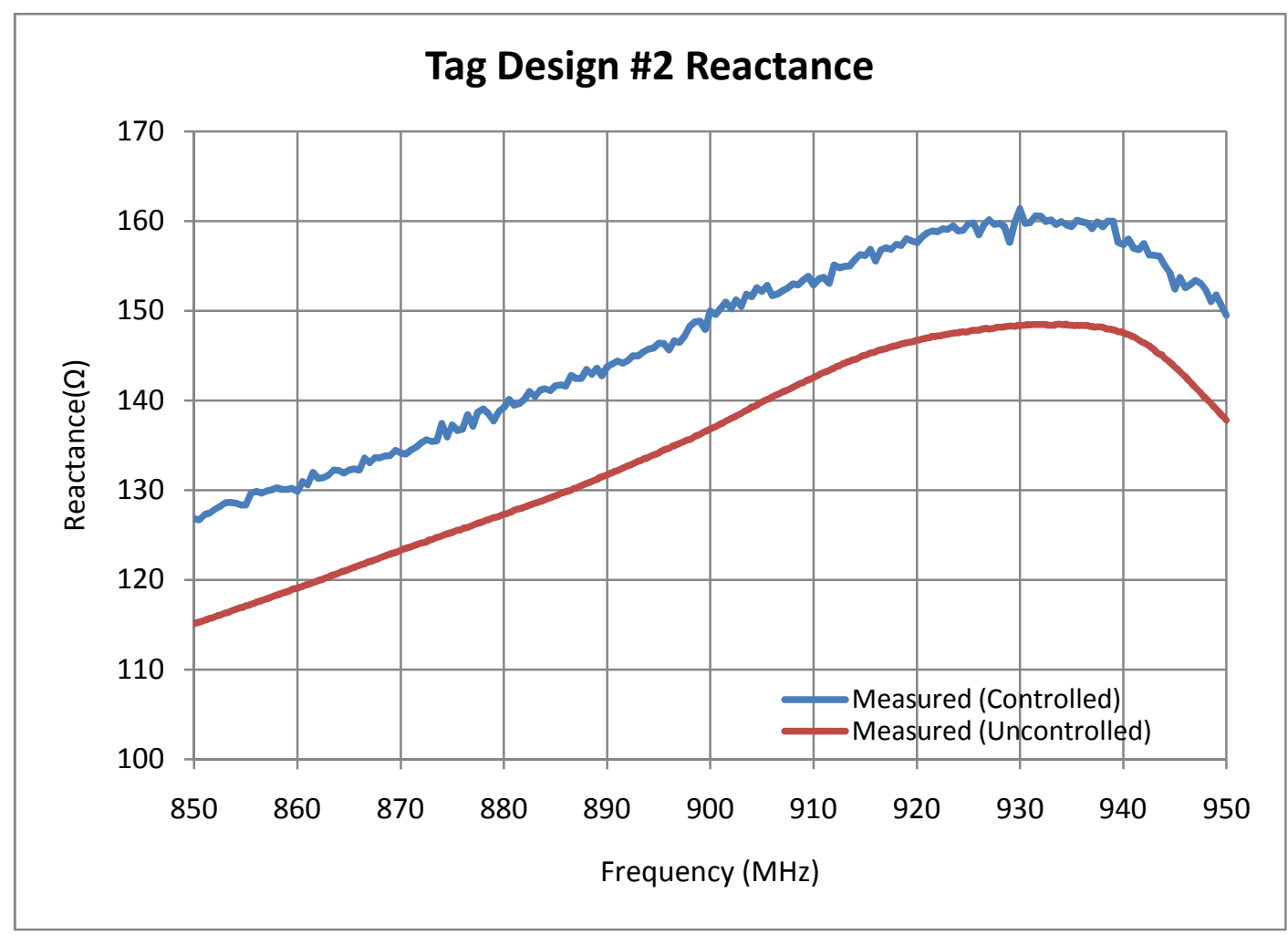

Figure 5.2: Measured RFID Antenna Reactance (Controlled vs. Uncontrolled Environment) 
3. Conduct patch antenna (TX/RX) gain measurements in the anechoic chamber to improve read range calculation accuracy.

4. Obtain accurate RFID IC input impedance values for complex conjugate match to the antenna.

5. Examine other RFID tag performance criteria, including reader scattering crosssection.

6. Investigate substrate and conductive materials effects on RFID performance (silver ink on PET vs. copper on FR4).

Figure 5.3 and Figure 5.4 show differences in EMPro simulated RFID antenna resistance and reactance frequency responses for PET and FR4 substrates.

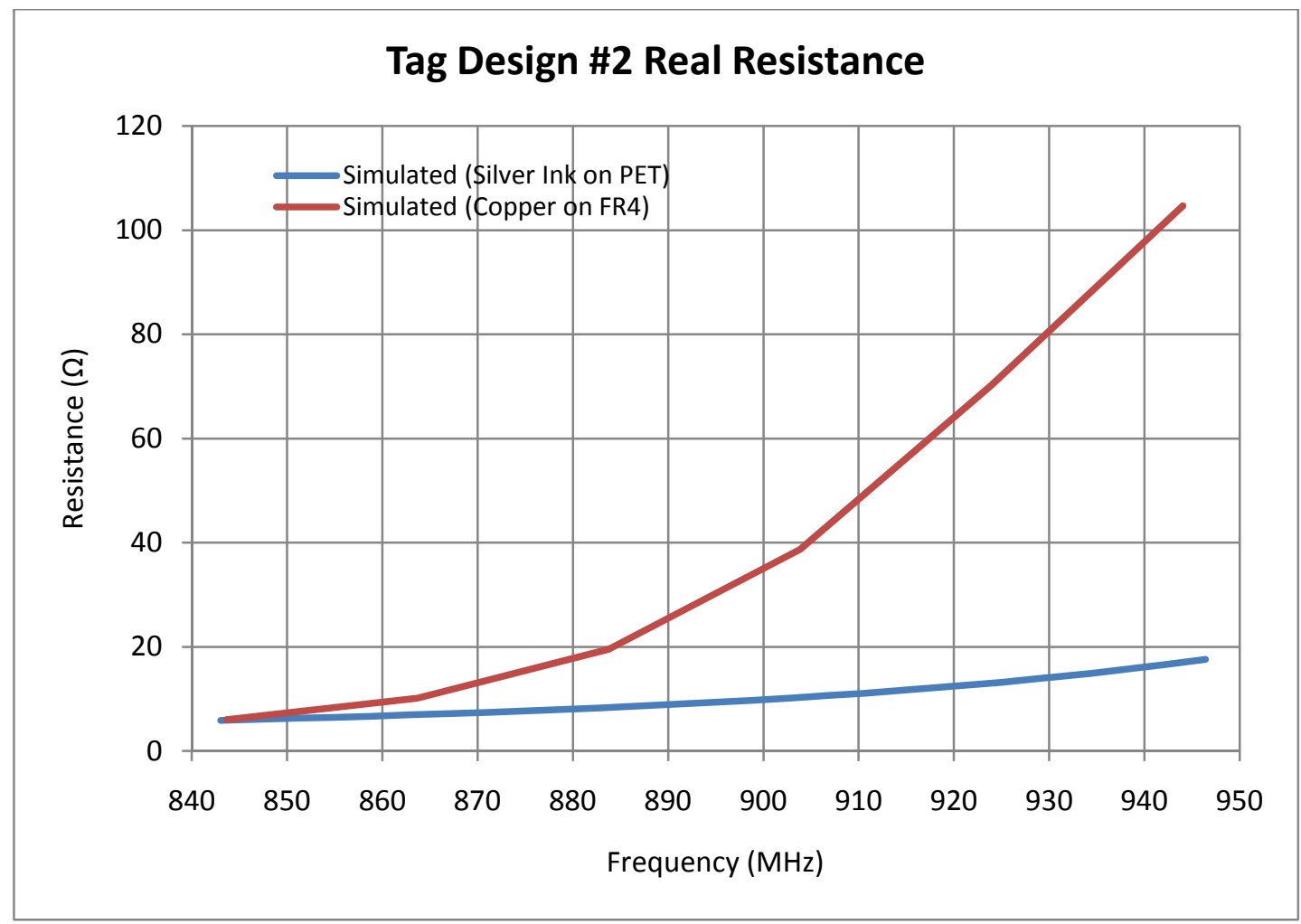

Figure 5.3: Simulated RFID Antenna Resistance, Silver Ink on PET vs. Copper on FR4 


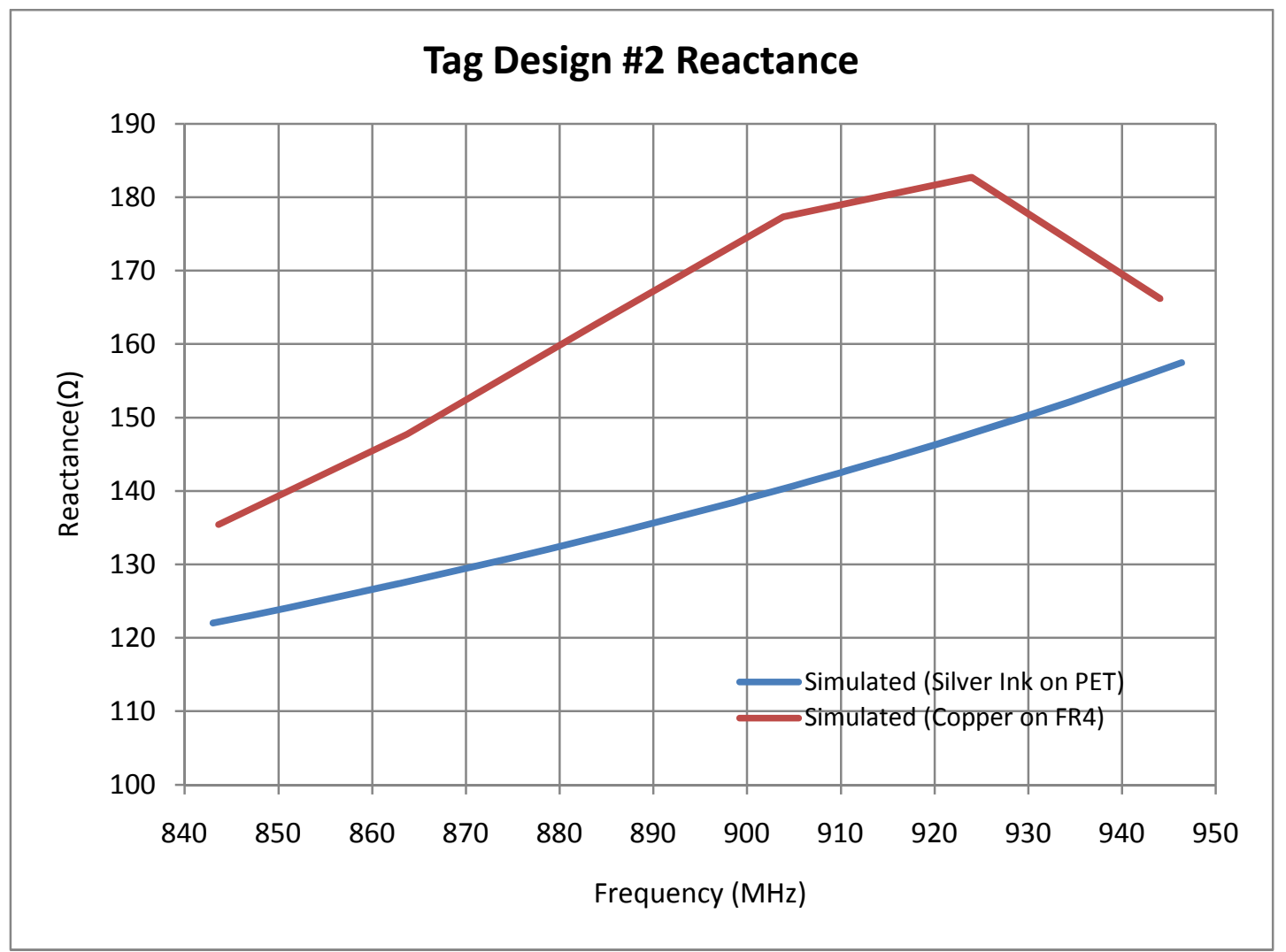

Figure 5.4: Simulated RFID Antenna Resistance, Silver Ink on PET vs. Copper on FR4 


\section{REFERENCES}

[1]. D. M. Dobkin, The RF in RFID: Passive UHF RFID in Practice. Newnes, 2007.

[2]. "Quick Introduction to RFID," PolyGAIT RFID Tutorial. PolyGAIT, Web. 14 Jan 2010. <http://www.polygait.calpoly.edu//tutorial.htm>.

[3]. "RFID Frequencies," ScanSource Europe Website, Available: $<$ www.scansource.eu/en/education.htm?eid=8\&elang=en>

[4]. "Asset Management Solution," RFIDHealthcare.com. BlueBean Company, Available Online, 14 Jan 2010. <http://www.rfidhealthcare.com/rfid-healthcareasset-management-solutions.aspx $>$.

[5]. A.R Koelle, S.W. Depp, and R.W. Freyman, "Short-range radio-telemetry for electronic identification, using modulated RF backscatter," Proceedings of the IEEE vol. 63, no. 8, pp. 1260-1261, Aug. 1975.

[6]. C. Mutti, C. Floerkemeier, "CDMA-based RFID Systems in Dense Scenarios: Concepts and Challenges," 2008 IEEE International Conference on RFID, pp. 215222, 16-17 April 2008

[7]. L. Wu, Y. Chen, C. Hung, and W. Kuo, "Zero-Collision RFID Tags Identification Based on CDMA," Information Assurance and Security, 2009. Fifth International Conference on IAS '09, vol. 1, pp. 513-516, 18-20 Aug. 2009.

[8]. C. A. Dabas, B. B. Mamta, and J.P. C. Gupta. "CDMA Based Anti-Collision Deterministic Algorithm for RFID Tags," International Journal of Recent Trends in Engineering, vol. 1, no. 1, pp. 603-606, May 2009.

[9]. M. Abbak, and I. Tekin, "RFID Coverage Extension Using Microstrip-Patch Antenna Array [Wireless Corner]," Antennas and Propagation Magazine, IEEE, vol.51, no.1, pp.185-191, Feb. 2009 Available:

$<$ http://www.ieeexplore.ieee.org/stamp/stamp.jsp?tp=\&arnumber=4939065\&isnum ber $=4939003$. $>$

[10]. H. Stockman, "Communication by Means of Reflected Power," Proceedings of the IRE , vol.36, no.10, pp. 1196-1204, Oct. 1948

[11]. Crump, "Folded Dipole Having a Direct Current Output," US Patent 2,943, 189, filed 1956, granted 1960.

[12]. Impinj Monza 3 Datasheet. Online: www.impinj.com/Documents/Tag_Chips/Monza_3_Tag_Chip_Datasheet/ 
[13]. Teijin® Tetoron ${ }^{\circledR}$ SL film (PET film) Datasheet. Online: http://www.dupontteijinfilms.com/filmenterprise/Datasheet.asp?ID=533\&Version= US

[14]. Spraylat XCSD-006 Polymer Thick Film Conductor Datasheet: Online: http://www.spraylat.com/Files/PTF\%20PDF/PDS-XCSD-006.pdf

[15]. Debatosh, Guha, and Y. M. Antar, Microstrip and Printed Antennas: New Trends, Techniques and Applications.UK: Wiley, 2011, p 265-303

[16]. A. M. Lyons, and D. W. Dahringer, "Electrically Conductive Adhesives." Handbook of Adhesive Technology. Taylor And Francis, Web. 22 July 2011. <http://203.158.253.140/media/e-

Book/Engineer/Material/Handbook\%20of\%20Adhesive\%20Technology/DK2131_ Ch45.pdf>.

[17]. E. M. Petrie, "Adhesive Technology in RFID Manufacture." Omnexus. SpecialChem, 8 Dec. 2010. Web. 22 July 2011.

<http://www.omnexus4adhesives.com/services/articles.aspx?id=3336>

[18]. Selector Guide for RFID Interconnect Adhesives. Online:

http://www.henkel.hu/huu/content_data/105682_RFID_SG_USletter.pdf

[19]. K.V.S. Rao, P.V. Nikitin, and S.F. Lam, "Antenna design for UHF RFID tags: a review and a practical application," IEEE Transactions on Antennas and Propagation, vol. 53, no. 12, pp. 3870- 3876, Dec. 2005

[20]. W. L. Stutzman, and G.A. Thiele, Antenna Theory and Design, 2nd edition, pp. 169-180.

[21]. EL-9032 Conductive Tape by Adhesive Research Inc Datasheet: Online: http://www.adhesivesresearch.com/Documents/Electronics\%20PI\%20Sheet/Electro nics\%20Market\%20PI\%20Sheets/9032\%20Data\%20Sheet.pdf

[22]. J. Choo, J. Ryoo, J. Hong, H. Jeon, C. Choi, and M.M. Tentzeris, "T-matching networks for the efficient matching of practical RFID tags," Microwave Conference, 2009. EuMC 2009. European , pp. 5-8, Sept. 29 2009-Oct. 12009

[23]. Sirit RSI-674 RFID Inlay Datasheet, Online: http://www.sirit.com/Inlay_Spec_Sheets/RSI-674.pdf

[24]. Y. T. Lo, and S.W. Lee, Antenna Handbook, Van Nostrand Reinhold, New York, pp. 27-21, 1988.

[25]. G. Marrocco, "The art of UHF RFID antenna design: impedance-matching and sizereduction techniques," IEEE Antennas and Propagation Magazine, vol. 50, no. 1, pp. 66-79, Feb. 2008 
[26]. H. W. Son, and C. S. Tyo, "Design of RFID Tag Antennas Using an Inductively Coupled Feed," Electronics Letters, vol. 45, no. 18, pp. 994-996, Sept. 2005.

[27]. N. A. Mohammed, K.R. Demarest, and D.D. Deavours, "Analysis and Synthesis of UHF RFID Antennas Using the Embedded T-match," 2010 IEEE International Conference on RFID, pp. 230-236, 14-16 April 2010

[28]. K. R. Demarest, and D. D. Deavours, "Limitations of the Uda Model for T-match Antennas," Progress In Electromagnetics Research, Vol. 113, pp. 1-15, 2011.

[29]. S. Uda, and Y. Mushiake, Yagi-Uda Antenna, Tokyo, Sasaki Printing and Publishing Co., 1954, pp. 119-131

[30]. C.A. Balanis, Antenna Theory Analysis and Design, New York: John Wiley \& Sons, 1997.

[31]. S. Chen, and K. Lin , "A folded dipole with a closed loop antenna for RFID applications," Antennas and Propagation Society International Symposium, 2007 IEEE, pp. 2281-2284, 9-15 June 2007

[32]. S. L. Chen, and K. H. Lin, "Performance of a Folded Dipole with a Closed Loop for RFID Applications," Progress In a Electromagnetic Research Symposium, Prague, Czech Republic, pp. 27-30 August 2007.

[33]. X. Qing, C. Goh, and Z. Chen, "Impedance Characterization of RFID Tag Antennas and Application in Tag Co-Design," IEEE Transactions on Microwave Theory and Techniques, vol. 57, no. 5, pp. 1268-1274, May 2009

[34]. R. Meys, and F. Janssens, "Measuring the impedance of balanced antennas by an Sparameter method," Antennas and Propagation Magazine, IEEE, vol. 40, no. 6, pp. 62-65, Dec 1998

[35]. H. Zhu, Y.C.A. Ko, and T.T. Ye, "Impedance measurement for balanced UHF RFID tag antennas," IEEE Radio and Wireless Symposium (RWS), pp. 128-131, 1014 Jan. 2010

[36]. X. Qing, C.K. Goh, and Z. Chen, "Measurement of UHF RFID tag antenna impedance," iWAT 2009. IEEE International Workshop on Antenna Technology, 2009, pp. 1-4, 2-4 March 2009

[37]. K.D. Palmer, and M.W. Rooyen, "Simple broadband measurements of balanced loads using a network analyzer," IEEE Transactions on Instrumentation and Measurement, vol. 55, no. 1, pp. 266- 272, Feb. 2006

[38]. S.K. Kuo, S.L. Chen, and C.-T. Lin, "An accurate method for impedance measurement of RFID tag antenna," Progress In Electromagnetics Research, vol. 83, pp. 93-106, 2008. 
[39]. M. Camp, R. Herschman, T. Zelder, and H. Eul, "Determination of the input impedance of RFID transponder antennas with novel measurement procedure using a modified on-wafer-prober." Advances in Radio Science, vol. 5, pp. 115-118, 2007

[40]. T. Koskinen, H. Rajagopalan, and Y.Rahmat-Samii, "Impedance measurements of various types of balanced antennas with the differential probe method," . iWAT 2009. IEEE International Workshop on Antenna Technology, 2009, pp. 1-4, 2-4 March 2009

[41]. X. Qing, and Z. Chen, "Comments on 'Measuring the Impedance of Balanced Antennas by an S-Parameter Method'," IEEE Antennas and Propagation Magazine , vol. 52, no. 1, pp. 171-172, Feb. 2010

[42]. T. Ogilvie, "RFID Tag Characterization," Technical Report to WaveZero, Inc., San Luis Obispo: Cal Poly, 2008.

[43]. "RFID Frequencies," ScanSource Europe Website, Available: $<$ www.scansource.eu/en/education.htm?eid=8\&elang=en>

[44]. N. Abramson, "Development of the ALOHANET,"IEEE Transactions on Information Theory, vol.31, no.2, pp. 119- 123, Mar 1985

[45]. T. Cheng, L. Jin, "Analysis and Simulation of RFID Anti-collision Algorithms," The 9th International Conference on Advanced Communication Technology, vol. 1, pp. 697-701, 12-14 Feb. 2007, Available:

$<$ http://www.ieexplore.iee.. rg/stamp/stamp.jsp?tp=\&arnumber=4195229\&isnum ber $=4195059$.>

[46]. Alien ALN-9800 Product Overview, Online: http://www.alientechnology.com/docs/products/DS_ALR-9800.pdf

[47]. EMPro 3D EM Simulation Software by Agilent Technologies Inc, Online: http://www.home.agilent.com/agilent/product.jspx?nid=$\underline{34278.0 .00 \& l \mathrm{l}=\mathrm{eng} \& \mathrm{cc}=\mathrm{US}}$

[48]. DMP-2831 Materials Inkjet Deposition System Datasheet, Online: http://www.dimatix.com/files/DMP-2831-Datasheet.pdf 


\section{APPENDIX A: RFID SYSTEM OPERATING FREQUENCY COMPARISON}

Table A-1: RFID System Frequency Band Classification [43]

\begin{tabular}{|c|c|c|c|c|c|}
\hline $\begin{array}{c}\text { Frequency } \\
\text { Band }\end{array}$ & Description & $\begin{array}{c}\text { Operating } \\
\text { Range }\end{array}$ & Applications & Benefits & Drawbacks \\
\hline $\begin{array}{l}125 \mathrm{kHz} \text { to } \\
134 \mathrm{kHz}\end{array}$ & $\begin{array}{l}\text { Low } \\
\text { Frequency } \\
\text { (LF) }\end{array}$ & $\begin{array}{l}<0.5 \mathrm{~m} \\
(1.5 \mathrm{ft})\end{array}$ & $\begin{array}{ll}- & \text { Access Control } \\
\text { - } & \text { Animal Tracking } \\
\text { - } & \text { Vehicle } \\
& \text { immobilizer } \\
\text { - } & \text { Product } \\
& \text { Authentication } \\
\end{array}$ & $\begin{array}{l}\text { Works well } \\
\text { around water } \\
\text { and metal } \\
\text { products }\end{array}$ & $\begin{array}{l}\text { Short read } \\
\text { range }\end{array}$ \\
\hline $13.56 \mathrm{MHz}$ & $\begin{array}{l}\text { High } \\
\text { Frequency } \\
\text { (HF) }\end{array}$ & $<1 \mathrm{~m}(3 \mathrm{ft})$ & $\begin{array}{ll}\text { - } & \text { Smart Cards } \\
\text { - } & \text { Smart shelf tags } \\
& \text { for item level } \\
& \text { tracking } \\
\text { - } & \text { Library Books } \\
\text { - } & \text { Airline Baggage } \\
\text { - } & \text { Maintenance } \\
& \text { data-logging }\end{array}$ & $\begin{array}{l}\text { Low cost } \\
\text { tags }\end{array}$ & $\begin{array}{l}\text { Short read } \\
\text { range }\end{array}$ \\
\hline $\begin{array}{l}860 \mathrm{MHz} \text { to } \\
930 \mathrm{MHz}\end{array}$ & $\begin{array}{l}\text { Ultrahigh } \\
\text { Frequency } \\
\text { (UHF) }\end{array}$ & $3 \mathrm{~m}(9 \mathrm{ft})$ & $\begin{array}{ll}\text { - } & \text { Pallet tracking } \\
\text { - } & \text { Carton Tracking } \\
\text { - } & \text { Electronic toll } \\
& \text { collection } \\
\text { - } & \text { Parking lot } \\
& \text { access } \\
\end{array}$ & $\begin{array}{l}\text { EPC } \\
\text { standard } \\
\text { developed } \\
\text { around this } \\
\text { frequency }\end{array}$ & $\begin{array}{l}\text { Does not work } \\
\text { well around } \\
\text { items of high } \\
\text { water or metal } \\
\text { content }\end{array}$ \\
\hline $2.4 \mathrm{GHz}$ & Microwave & $1 \mathrm{~m}(3 \mathrm{ft})$ & $\begin{array}{ll}\text { - } & \text { Airline Baggage } \\
\text { - } & \text { Electronic toll } \\
\text { collection }\end{array}$ & $\begin{array}{l}\text { Fastest read } \\
\text { rates }\end{array}$ & $\begin{array}{l}\text { Most } \\
\text { expensive }\end{array}$ \\
\hline
\end{tabular}

Note: Read range may vary due to environment conditions, tag mounting surface, and external interference sources. The above ranges are given as guidelines [43]. 
Table A-2: RFID Tag Powering Classification [1]

\begin{tabular}{|c|c|c|c|c|c|}
\hline Description & Features & $\begin{array}{c}\text { Operating } \\
\text { Range }\end{array}$ & Applications & Benefits & Drawbacks \\
\hline Passive Tag & $\begin{array}{l}\text { No } \\
\text { independent } \\
\text { power } \\
\text { source and } \\
\text { no radio } \\
\text { transmitter }\end{array}$ & $3 \mathrm{~m}(9 \mathrm{ft})$ & $\begin{array}{ll}\text { - } & \text { Inventory and } \\
\text { low value } \\
\text { asset tracking } \\
\text { - Animal } \\
\text { Tracking }\end{array}$ & $\begin{array}{l}\text { Simple circuitry, } \\
\text { small size, low } \\
\text { cost, and low } \\
\text { maintenance }\end{array}$ & $\begin{array}{l}\text { Limited read } \\
\text { range; limited RF } \\
\text { communication } \\
\text { capacity and link } \\
\text { quality; limited } \\
\text { security and } \\
\text { privacy; Sensitive } \\
\text { to environment; } \\
\text { Tag cost as low as } \\
\$ 0.01 \text { each. }\end{array}$ \\
\hline $\begin{array}{l}\text { Semipassive } \\
\text { Tag }\end{array}$ & $\begin{array}{l}\text { Independent } \\
\text { power } \\
\text { source and } \\
\text { no radio } \\
\text { transmitter }\end{array}$ & $\begin{array}{l}100 \mathrm{~m} \\
(328 \mathrm{ft})\end{array}$ & 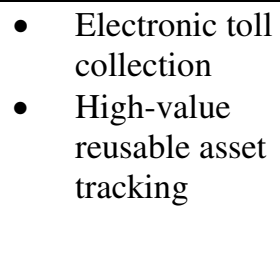 & $\begin{array}{l}\text { More responsive } \\
\text { to valid } \\
\text { interrogation. } \\
\text { More reliable. } \\
\text { Use of standard } \\
\text { commercial ICs. }\end{array}$ & $\begin{array}{l}\text { Increase in size, } \\
\text { cost and } \\
\text { maintenance } \\
\text { requirements. Tag } \\
\text { cost US } \$ 20-30 \\
\text { each with } 5 \text { year } \\
\text { battery cycle. }\end{array}$ \\
\hline Active Tag & $\begin{array}{l}\text { Independent } \\
\text { power } \\
\text { source with } \\
\text { full-fledged } \\
\text { radios }\end{array}$ & $\begin{array}{l}\text { Up to } 1 \mathrm{~km} \\
(0.62 \\
\text { miles })\end{array}$ & $\begin{array}{ll}\text { - } & \text { Large Assets } \\
& \text { Tracking }\end{array}$ & $\begin{array}{l}\text { More } \\
\text { sophisticated and } \\
\text { effective radio } \\
\text { communication. } \\
\text { Superior noise } \\
\text { robustness. } \\
\text { Robust against } \\
\text { environment }\end{array}$ & $\begin{array}{l}\text { Additional } \\
\text { increase in size, } \\
\text { cost and } \\
\text { maintenance } \\
\text { requirements of } \\
\text { the radios. } \\
\text { Regulatory } \\
\text { Standard required } \\
\text { Tag cost about } \\
\text { US } \$ 50 \text { each in } \\
\text { large quantities } \\
\text { with } 6 \text { year } \\
\text { battery cycle. }\end{array}$ \\
\hline
\end{tabular}




\section{APPENDIX B: UHF RFID SYSTEM PROTOCOLS AND ANTI- COLLISION ALGORITHMS}

UHF passive tag Radio-Frequency Identification (RFID) systems have read ranges up to $3 \mathrm{~m}$. Increased read range allows additional tags to enter into a reader's interrogation zone. When all tags transmit simultaneously, mutual interference can occur and cause data loss. This event is referred to as a collision. To address this problem, anticollision algorithms were developed for different UHF RFID Reader Protocols. Table B-1 provides a summary of anti-collision algorithms used in conjunction with multiple UHF RFID Reader Protocols.

Table B-1: RFID Reader Protocols and Anti-Collision Algorithms

\begin{tabular}{|l|l|}
\hline \multicolumn{1}{|c|}{ RFID Reader Protocol } & \multicolumn{1}{c|}{ Anti-Collision Algorithm } \\
\hline EPC Class1 & Bin-based Binary Tree Algorithm \\
\hline $\begin{array}{l}\text { EPC Class1 Generation 2 (abbreviate as } \\
\text { Gen2) }\end{array}$ & "Q"-protocol based on DFSA \\
\hline ISO 18000-6 Type A & DFSA Algorithm \\
\hline ISO 18000-6 Type B & Binary Tree Algorithm \\
\hline
\end{tabular}

The anti-collision algorithms specified in Table B-1 are derived from two widelyused algorithms - Framed Slotted ALOHA (FSA) and Binary Tree Algorithm.Both methods are based on TDMA (time division multiple access). ALOHA, also known as ALOHAnet, [44] is a pioneering computer networking system developed at the University of Hawaii in 1970s. The project developed a radio linked computer network which connects different college campus for computer resources sharing. TDMA allows users to sequentially access a single radio frequency. In the FSA algorithm, each reader command is divided into multiple frames each consisting of several slots. Tags generate a random number that is used to select a slot in one frame; the tag responds only in its selected slot. The reader identifies the tag when there is an only one tag response in one slot. Dynamic Framed Slotted ALOHA (DFSA) changes the frame size dynamically 
during tag identification [45]. The Binary Tree Algorithm is based on the model shown in Figure B.1. The reader first divides the tags into two subsets ( $\mathrm{s} 0$ and $\mathrm{s} 1$ ) and queries s0 and s1 in sequence. Whenever collisions occur, tags within the subset are further divided into additional subsets (for example, s10 and s11 in Figure B.1). The subset division process repeats until no collisions are detected. Subsets s100 and s101 in Figure B.1 indicate that all the tags are correctly detected.

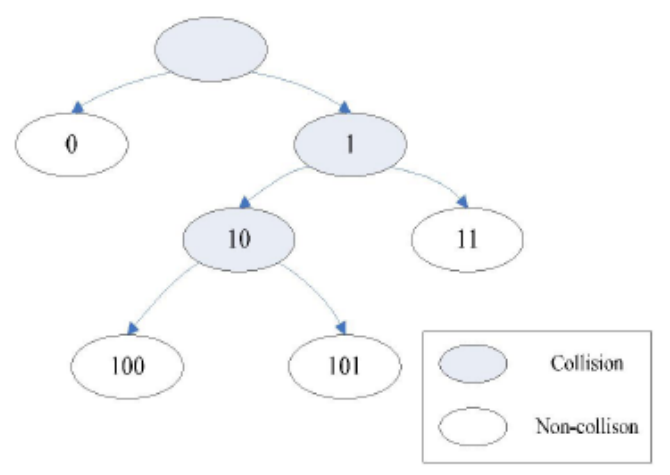

Figure B.1: Binary Tree Model [45]

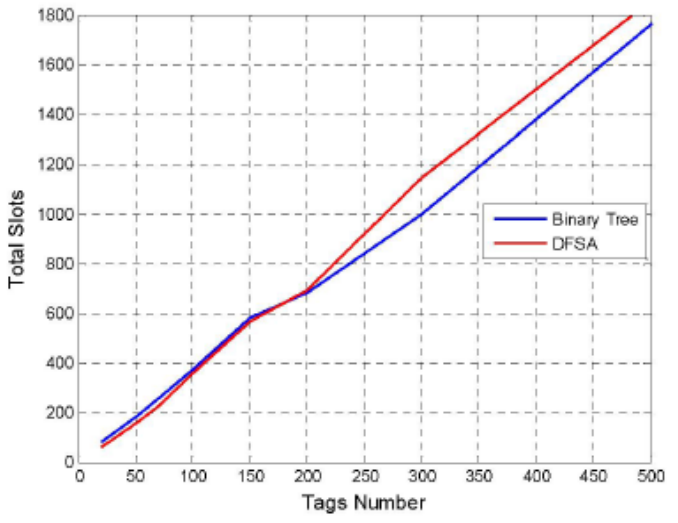

Figure B.2: Binary Tree and DFSA Algorithm Comparison [45]

For both algorithms, the total number of slots is proportional to the number of tags as shown in Figure B.2 [45]. 


\section{APPENDIX C: RFID ANTENNA FABRICATION TECHNIQUES [15]}

\begin{tabular}{|c|c|c|c|}
\hline Technique & Description & Benefits & Drawback \\
\hline $\begin{array}{l}\text { Plating and } \\
\text { Etching }\end{array}$ & $\begin{array}{l}\text { Uses conventional } \\
\text { photolithography and etching of } \\
\text { electroplated copper. }\end{array}$ & $\begin{array}{l}\text { - Excellent results } \\
\text { - Standard in industry } \\
\text { for decades } \\
\text { - Useful for short } \\
\text { production runs of } \\
\text { tags that require high } \\
\text { conductivity or } \\
\text { specialty tags. }\end{array}$ & $\begin{array}{l}\text { - Awkward to dispose } \\
\text { in environmentally- } \\
\text { acceptable methods } \\
\text { Wastes expensive } \\
\text { copper }\end{array}$ \\
\hline $\begin{array}{l}\text { Silver Ink } \\
\text { Screen Printing }\end{array}$ & $\begin{array}{l}\text { Silver inks contain small silver } \\
\text { particles suspended in a } \\
\text { solution. Ink application is } \\
\text { possible by silk screen, and ink } \\
\text { jet printing. }\end{array}$ & $\begin{array}{l}\text { - Easy to use } \\
\text { - Does not require large } \\
\text { expensive equipment } \\
\text { and chemicals }\end{array}$ & 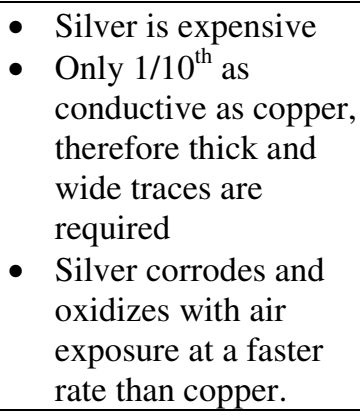 \\
\hline $\begin{array}{l}\text { Vapor } \\
\text { Deposition }\end{array}$ & $\begin{array}{l}\text { Metal deposition via } \\
\text { evaporation performed at low } \\
\text { pressures }\left(<10^{-5} \text { Torr }\right) \text {. } \\
\text { Antenna is formed by } \\
\text { evaporating aluminum through } \\
\text { a shadow mask. }\end{array}$ & $\begin{array}{l}\text { - Inexpensive in large } \\
\text { batches due to single } \\
\text { deposition step. }\end{array}$ & $\begin{array}{l}\text { Metal accumulates } \\
\text { on the shadow mask, } \\
\text { requiring periodic } \\
\text { chamber cleaning. }\end{array}$ \\
\hline $\begin{array}{l}\text { Deposition and } \\
\text { Laser Ablation }\end{array}$ & $\begin{array}{l}\text { A thin copper or aluminum } \\
\text { layer on a polyester substrate is } \\
\text { vaporized off the surface using } \\
\text { a high powered laser. }\end{array}$ & $\begin{array}{l}\text { - Highly automated } \\
\text { - Custom fabrication }\end{array}$ & $\begin{array}{l}\text { Requires special } \\
\text { infrastructure and } \\
\text { high quality, uniform } \\
\text { metal layers } \\
\text { - Slow } \\
\text { - Inappropriate for } \\
\text { high-volume, low } \\
\text { cost fabrication } \\
\end{array}$ \\
\hline $\begin{array}{l}\text { Printing and } \\
\text { Plating }\end{array}$ & $\begin{array}{l}\text { The electroplating process } \\
\text { starts with thin conductive layer } \\
\text { deposition onto polyester film. } \\
\text { This film is dipped into a } \\
\text { chemical bath containing } \\
\text { copper sulfate, and a negative } \\
\text { voltage is applied to the } \\
\text { conductive layer. The copper is } \\
\text { selectively deposited onto the } \\
\text { conductive region to form an } \\
\text { antenna. }\end{array}$ & & \\
\hline $\begin{array}{l}\text { Electroless } \\
\text { (Chemical) } \\
\text { Deposition }\end{array}$ & $\begin{array}{l}\text { A special catalyst ink pattern, } \\
\text { followed by a curing step. The } \\
\text { pattern is then placed in a } \\
\text { chemical bath containing an } \\
\text { electroless plating solution. } \\
\text { Once the process starts, metal } \\
\text { layers are formed without the } \\
\text { need for an external current } \\
\text { source. }\end{array}$ & $\begin{array}{l}\text { - Slightly less } \\
\text { expensive than the } \\
\text { selective } \\
\text { electroplating process, } \\
\text { such as printing and } \\
\text { plating. }\end{array}$ & $\begin{array}{l}\text { - Metal deposition rate } \\
\text { is slow, which } \\
\text { increases the time } \\
\text { needed in the } \\
\text { solution; which } \\
\text { increases cost. }\end{array}$ \\
\hline
\end{tabular}




\begin{tabular}{|c|c|c|c|}
\hline Die Cut & $\begin{array}{l}\text { A thin layer of copper or } \\
\text { aluminum is first formed on } \\
\text { polyester film using vapor } \\
\text { deposition or adhesion. The } \\
\text { polyester side is then laminated } \\
\text { with a thin layer adhesive and a } \\
\text { release liner. A rotary die } \\
\text { cutter, laser, or some other } \\
\text { means can be used to cut the } \\
\text { antenna pattern. After stripping } \\
\text { the unwanted metalized } \\
\text { polyester away, the antenna } \\
\text { remains. }\end{array}$ & $\begin{array}{l}\text { - Can be used to make a } \\
\text { "quick and dirty" } \\
\text { antenna using copper } \\
\text { foil, a design printed } \\
\text { on a label, a sharp } \\
\text { knife and a straight } \\
\text { edge. } \\
\text { - Fairly inexpensive } \\
\text { and fast process using } \\
\text { established roll-to-roll } \\
\text { processes. }\end{array}$ & $\begin{array}{ll}\text { - } & \text { Limited resolution } \\
\text { - } & \text { IC must be mounted } \\
\text { on a strap }\end{array}$ \\
\hline
\end{tabular}




\section{APPENDIX D: SCREEN PRINTING FILE FORMATS}

The following procedure is used to transfer EMPro files to DWF/DXF format, which can be processed for screen-printing procedure. These procedures are developed for EMPro version 2011.04 and ADS 2009.

1) Delete the air box and port setup (including coax lines) in the EMPro design; these are not needed for fabrication. Keep the substrate and signal layers.

2) Export the design: File > Export Parts...(EMPro 2011.04)

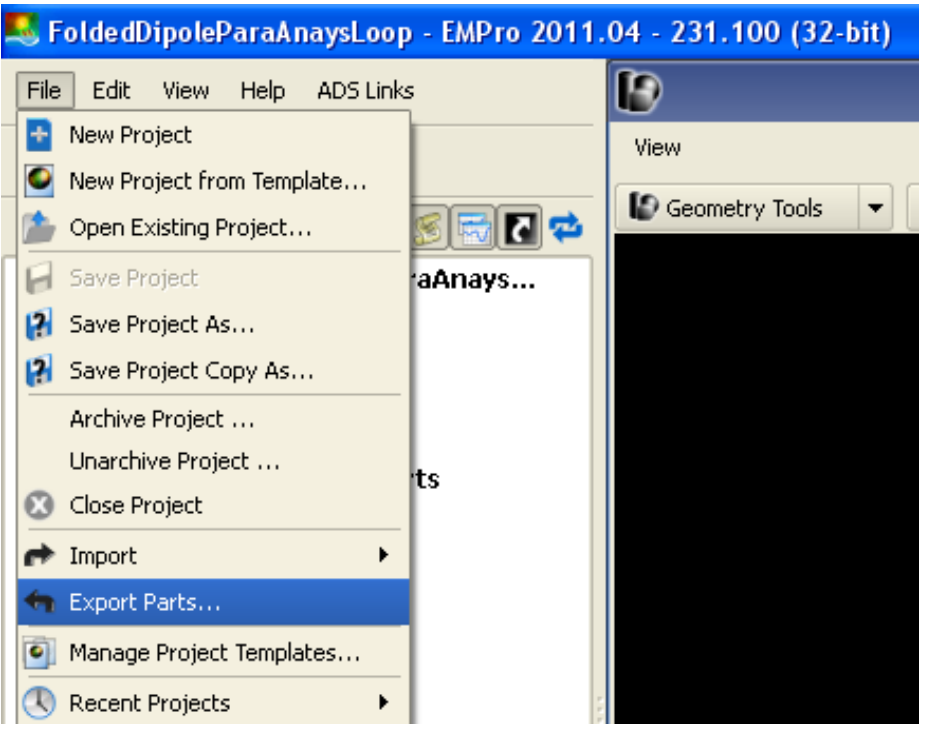

3) Save the design as an IGES file. One file will be created: *.igs in your specified directory

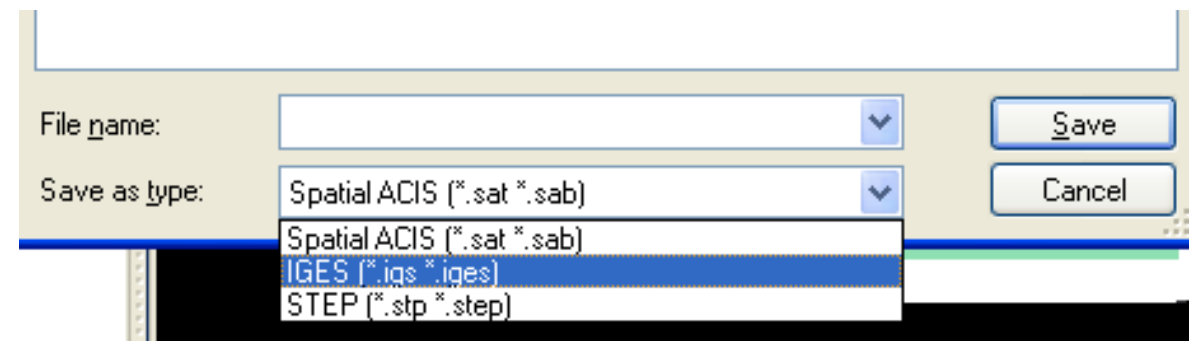

4) Open Advanced Design System 2009 (ADS) and create a new project

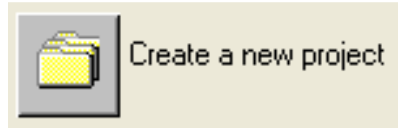

5) Select units consistent with the EMPro file 


\begin{tabular}{|c|c|c|}
\hline New Project & & \\
\hline \multicolumn{3}{|l|}{ Name- } \\
\hline C:'users'default' & & Browse... \\
\hline \multicolumn{3}{|l|}{ Project Technology Files:- } \\
\hline ADS Standard: Length unit--millimeter & $\nabla$ & View Details... \\
\hline \multicolumn{3}{|l|}{ ADS Standard: Length unit--mil } \\
\hline $\begin{array}{l}\text { ADS Standard: Length unit--millimeter } \\
\text { ADS Standard: Length unit--micron }\end{array}$ & & \multirow[t]{2}{*}{ Help } \\
\hline T〈Add Custom Technology〉 & & \\
\hline
\end{tabular}

6) Open New Layout Window: In the Main window, select New Layout Window Icon in the menu pictured below as indicated by the red box:

\begin{tabular}{l} 
EF Advanced Design System 2009 (Main) \\
File View Tools Window Designkit DesignGuide Help \\
\hline File View
\end{tabular}

7) Import *.igs file: In the Layout Window, File > Import...

When the Import window opens, select file type IGES and locate the desired file. Click OK, Ignore the warning window (Click OK)

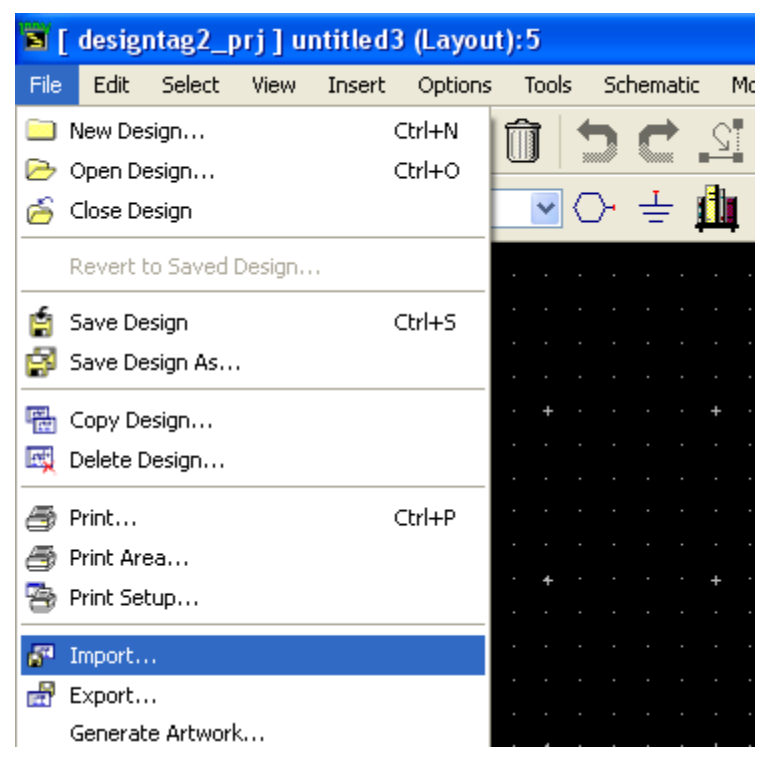

The example imported image is shown below 


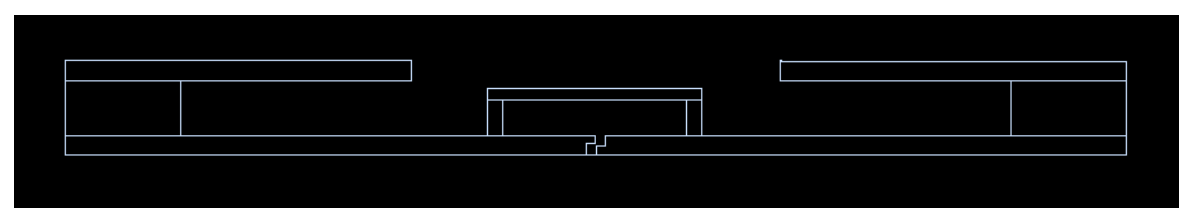

8) In Layout Window, export to DXF/DWG format: File > Export...

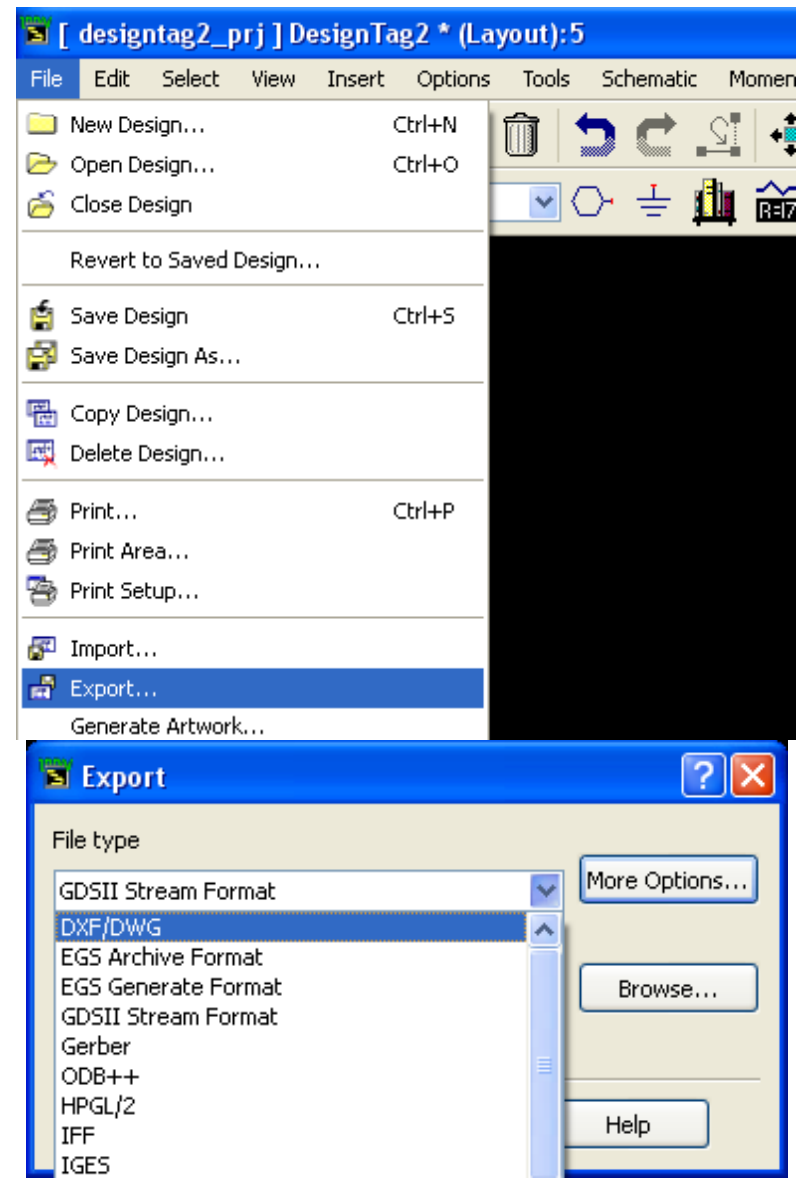

In the Export window, select DXF/DWG as file type, then, click OK

In the specified directory, a file with .dxf file is created. DXF/DWG file is AutoCAD file that can be further processed to create the screen printing screen. 